\title{
Fast compensatory functional network changes caused by reversible inactivation of monkey parietal cortex.
}

\section{Abbreviated title: Functional reorganization caused by LIP inactivation}

Puiu F. Balan*1, Annelies Gerits ${ }^{\star 1}$, Qi Zhu ${ }^{1}$, Hauke Kolster ${ }^{1}$, Guy A. Orban ${ }^{1,3}$, Claire Wardak $^{1}$, Wim Vanduffel ${ }^{1,2,4}$

${ }^{*}$ Contributed equally

1: Laboratorium voor Neuro- en Psychofysiologie, KU Leuven Medical School, Campus Gasthuisberg, 3000 Leuven, Belgium

2: Athinoula A. Martinos Center for Biomedical Imaging, Massachusetts General Hospital, Charlestown, Massachusetts, USA 02129.

3: Department of Medicine and Surgery, University of Parma, via Volturno 39E Parma, Italy

4: Department of Radiology, Harvard Medical School, Charlestown, Massachusetts, USA 02129.

Corresponding author: W. Vanduffel, Athinoula A. Martinos Center for Biomedical Imaging, Massachusetts General Hospital, 149 Thirteenth street, Charlestown, Massachusetts, USA 02129 Wim@nmr.mgh.harvard.edu 


\section{Abstract}

The brain has a remarkable capacity to recover after lesions. However, little is known about compensatory neural adaptations at the systems level. We addressed this question by investigating behavioral and (correlated) functional changes throughout the cortex that are induced by focal, reversible inactivations. Specifically, monkeys performed a demanding covert spatial attention task while the lateral intraparietal area (LIP) was inactivated with muscimol and whole-brain fMRI activity was recorded. The inactivation caused LIP-specific decreases in task-related fMRI activity. In addition, these local effects triggered large-scale network changes. Unlike most studies in which animals were mainly passive relative to the stimuli, we observed heterogeneous effects with more profound muscimol-induced increases of task-related fMRI activity in areas connected to LIP, especially FEF). Furthermore, in areas such as FEF and V4, muscimol-induced changes in fMRI activity correlated with changes in behavioral performance. Notably, the activity changes in remote areas did not correlate with the decreased activity at the site of the inactivation, suggesting that such changes arise via neuronal mechanisms lying in the intact portion of the functional task network, with FEF a likely key player. The excitation-inhibition dynamics unmasking existing excitatory connections across the functional network might initiate these rapid adaptive changes.

Keywords: attention network, fMRI, LIP, muscimol, visual search 


\section{Introduction}

Focal brain damage is usually followed by extensive structural and functional changes in non-damaged brain regions. It is generally assumed that recruitment of these regions outside the lesion underlies functional recovery (Ward NS et al. 2003). It is still a matter of debate, however, whether behavioral recovery after a lesion should be attributed to reduced (Marshall RS et al. 2000; Calautti C et al. 2001; Ward NS et al. 2003) or increased activity (Chollet F et al. 1991; Weiller C et al. 1992; Seitz RJ et al. 1998; Cramer SC et al. 2001) in areas at a distance from the lesion. Reversible deactivation experiments in animals combined with measurements of either metabolic or neuronal activity have revealed mainly reduced activity in sites connected with the deactivated cortex (Alonso JM et al. 1993; Vanduffel W et al. 1997; Hupe JM et al. 1998; Ponce CR et al. 2008). This is predictable since long-range cortico-cortical connections are mainly excitatory in nature (Salin PA and J Bullier 1995). Therefore, eliminating these connections from their input should lead, at least initially, to reduced excitation - hence decreased neuronal activity in remote sites. However, this also depends on the types of neurons (excitatory/inhibitory) that receive the excitatory input in connected sites. Moreover, such changes may also depend on the state and task demands of the subjects. Indeed, it can be argued that networks involved in specific perceptual or behavioral tasks should increase their activities in those network-nodes unaffected by the lesion, to actively compensate for the loss of functionality in the lesioned nodes.

Previous studies of cortical functional reorganizations in animals were unable to resolve this conundrum since subjects were mostly passive relative to sensory stimulation (Alonso JM et al. 1993; Vanduffel W et al. 1997; Hupe JM et al. 1998; Payne 
BR and SG Lomber 1999; Lomber SG et al. 2007; Ponce CR et al. 2008). A notable exception is the Wilke et al. study (Wilke M et al. 2012), in which monkeys performed a simple saccade task while LIP was reversibly inactivated during fMRI. This study largely confirmed the passive sensory stimulation studies since most sites connected with LIP showed decreased fMRI activity.

To investigate in more detail the task-relevance of functional changes observed immediately after reversible inactivation, we trained two monkeys on a demanding cognitive task with a manual response as operant behavior. Specifically, the animals were trained to perform a conjunction search task and a peripheral detection task (Fig. 1).

These cognitive tasks activated functional networks including frontal, parietal and occipital visual cortical areas. Beyond the obvious local decrease in activity, inactivation of LIP caused mainly increased fMRI activity in a large number of remote cortical sites. Moreover, we found that, in some parts of the task-related network (especially in FEF and V4), inactivation resulted in an increased correlation between behavior of the animal and fMRI activity. Our results suggest that a small reversible lesion in a critical node triggered rapid adaptive functional mechanisms within the functional network integrating that specific node.

\section{Materials and Methods}

Two male rhesus monkeys (Macaca Mulatta, M9 and M10, 4.5-6 kg, 5-6 years old) participated in this study. Animal care and experimental procedures met the national and European guidelines and were approved by the ethical committee of the KU 
Leuven. Animal housing and handling were in accordance with the recommendations of the Weatherall report, allowing extensive locomotor behavior, social interactions and foraging. All animals were group-housed (cage size at least $16-32 \mathrm{~m}^{3}$ ) with cage enrichment (toys, foraging devices) at the primate facility of the KU Leuven Medical School. They were daily fed with standard primate chow supplemented with bread, nuts, raisins, prunes and fruits. The animals received their daily water supply during the experiments until satiated.

Each monkey was implanted with an MR-compatible plastic headset attached to the skull with ceramic screws and acrylic. An MR-compatible plastic chamber (Crist Instrument, Hagerstown, MD) was placed above the parietal sulcus using a Brainsight ${ }^{\mathrm{TM}}$ neuro-navigation system (9 $\mathrm{mm}$ posterior, $13 \mathrm{~mm}$ lateral for $\mathrm{M} 9$ and $3 \mathrm{~mm}$ posterior, 16 $\mathrm{mm}$ lateral for M10). The grid was aligned approximately parallel to the curvature of the sulcus.

All operations were performed under isoflurane $(1.5 \%) / \mathrm{N}_{2} \mathrm{O}(50 \%) / \mathrm{O}_{2}(50 \%)$. Antibiotics (50 mg/kg i.m., Kefzo ${ }^{\circledR}$, Lilly, Brussels) and analgesics (4 mg/kg, i.m., Metacam $^{\circledR}$, Ingelheim/Rhein, Germany) were given daily for 3-7 days following each surgery (Vanduffel W et al. 2001).

\section{Visual stimuli and main tasks}

The monkeys performed two cognitive tasks (Fig. 1): a visual conjunction search task referred to as $\mathrm{CJ}$, and a peripheral 'brightening' detection task adapted to match the difficulty of conjunction trials, referred to as DET (Wardak C et al. 2010). In addition, the monkeys performed a baseline visual fixation task. Averaged over time, visual 
stimulation was exactly matched between the $\mathrm{CJ}$ and the DET task (in one set of runs) except for the color of the fixation point.

\section{Tasks}

In the $\mathbf{C} \mathbf{J}$ task, the monkey had to detect the presence of a predefined target presented among distracters $\left(2,4\right.$ or 8 items in total; Fig. $1 \mathrm{~A}$, left). The target was a $3^{\circ} \times 0.5^{\circ}$ blue bar, $45^{\circ}$ tilted clockwise from vertical. The distracters were $3^{\circ} \times 0.5^{\circ}$ red bars tilted $45^{\circ}$ clockwise or counter-clockwise, and $3^{\circ} \times 0.5^{\circ}$ blue bars tilted $45^{\circ}$ counter-clockwise from vertical.

Luminance levels of the stimuli were measured every scanning session using a Minolta CS-100 Chroma meter (Mayer International Ltd, UK). Target and distracters were presented on a gray background with a mean luminance of $9.4 \pm 0.9 \mathrm{~cd} / \mathrm{m}^{2}$. We presented isoluminant targets and distracters so that during search the monkey could rely on orientation and color but not the luminance of the target. The ratio of the red versus blue luminance was $5.69 \pm 0.21$, corresponding to perceived isoluminance between red and blue in the macaque monkey (Tootell RB et al. 2004).

In the DET task the monkey had to detect a portion of the background that changed in luminance (see Wardak et al. (2010) for details). This portion was an area defined by $\pi / 9$ rad arcs of two concentric circles with radii of $5.7^{\circ}$ and $8.3^{\circ}$ respectively (thus it covered the same spatial location as the bars; Fig. 1A, right).

To equalize overall visual stimulation during the $\mathrm{CJ}$ and the DET tasks, the taskrelevant events (bar stimuli for $\mathrm{CJ}$, and brightening for DET) were also presented in the 
other task), although they occurred at different timings within a trial and were irrelevant for solving the task (Fig. 1B and C).

Notably, during the $\mathrm{CJ}$ and DET tasks, the monkeys had to simultaneously attend both hemifields in order to detect the respective targets. Also, it needs to be noted that the monkeys did not have to indicate the position of the target (e.g. in the left or right hemifield), only its presence.

\section{Position of the stimuli}

Two, four, or eight bars were presented along a circular path of $7^{\circ}$ radius. When 8 bars were shown, the objects were positioned every $\pi / 4$ radians starting at the $\pi / 8$ location. In this way 2 bars were located in each quadrant and none on the meridians (Fig. 1). In the 2- and 4-item conditions the bars randomly occupied 2 and 4 of the 8 predefined locations. All positions were equally occupied over time and the target was presented equally in each of these 8 locations. To avoid visual adaptation, a small jitter was introduced at each of the 8 positions $\left(\sim 0.5^{\circ}\right.$ change of position in the vertical or the horizontal direction at every object presentation, but balanced on average).

The location of the change in background luminance was pseudo-randomly chosen from the 8 possible locations occupied by the bars, and the same number of brightening events occurred at each location (Fig. 1A, right).

\section{CJ trials}


During CJ trials, a dark red fixation point $\left(0.2^{\circ} \times 0.2^{\circ}\right)$ was always present in the center of the screen. The monkey was required to continuously maintain fixation within a $2^{\circ} \times 3^{\circ}$ window centered on the fixation point to avoid aborting the trial.

After 100-200 ms of fixation, the bars were presented for 1300 ms (Fig. 1B; grey part of timeline). A target was present in half the trials during which the monkey had to interrupt an infrared light path by lifting the right hand to be rewarded after the stimulus ended. The response window started $100 \mathrm{~ms}$ after target appearance and lasted until stimulus end (1200 ms in total). If the monkey did not respond within this response window when a target was present, the trial was scored incorrect. If no target was present (other $50 \%$ of the trials) the monkey was rewarded after stimulus end for not responding. When the monkey made a response before or after the response window during any trial, it was aborted.

To equalize total visual stimulation between CJ and DET epochs, a portion of the grey background luminance was changed during the last $300 \mathrm{~ms}$ of the stimulus duration in half of the $\mathrm{CJ}$ trials. This event had to be ignored by the monkey during the CJ trials.

The interval between two stimulus presentations, i.e. when no stimulus was presented, lasted randomly between 1000 and $1250 \mathrm{~ms}$ (Fig. 1B). Throughout this interstimulus-interval the fixation point remained present. The monkey had to keep fixating to avoid a delay (between $700-1000 \mathrm{~ms}$ ) of the next trial.

\section{DET trials}


The DET trials were identical to the CJ trials except for the green $\left(0.2^{\circ} \times 0.2^{\circ}\right.$ square $)$ fixation point and timing of the luminance change in the background, which started between 50 and $400 \mathrm{~ms}$ after bar appearance and lasted for $300 \mathrm{~ms}$ (see Fig. 1C). The monkey was required to continuously maintain fixation within a $2^{\circ} \times 3^{\circ}$ window centered on the fixation point to avoid aborting the trial. During detection trials (DET) the monkeys were rewarded for indicating, with a manual response, a luminance change that was present in $50 \%$ of the trials. The subjects were also rewarded for not responding during the other $50 \%$ of trials without luminance change. During the detection trials, the subjects had to ignore the presence of the blue items tilted $45^{\circ}$ clockwise from vertical (i.e. the 'targets' during the CJ trials).

DET trials, as were the CJ trials, were rewarded when the monkey responded correctly, after stimulus end (Fig. 1).

\section{Fixation trials}

A fixation condition was used as a baseline during the fMRI scans. In this condition, only a light red fixation point $\left(0.2^{\circ} \times 0.2^{\circ}\right.$ square $)$ was continuously present (Fig. 1D). To be rewarded, the monkey had to maintain fixation within a $2^{\circ} \times 3^{\circ}$ window centered on the point. The monkey was rewarded at the same rate as during the $\mathrm{CJ}$ and the DET task, averaging a reward every $2400 \mathrm{~ms}$. Owing to the extensive training, both monkeys fixated accurately. In all tasks, the fixation behavior declined only when subjects were satiated.

\section{fMRI Scanning}


Monkeys sat in a sphinx position in a plastic monkey chair placed within the horizontal bore of the magnet (3 T MR scanner Sonata; Siemens, Erlangen, Germany) directly facing a translucent screen placed $57 \mathrm{~cm}$ from the eyes. Visual stimuli were rearprojected onto this screen using a Barco RLM Performer R6+ projector (1400 x 1050 pixels, $60 \mathrm{~Hz}$ ) (Vanduffel et al., 2001).

The head was restrained by means of the MR-compatible headpost. An 8channel phased-array receive coil was positioned closely around its head (Ekstrom LB et al. 2008). However, due to the presence of a unilaterally positioned recording chamber (left for M9, right for M10), the coil was positioned at a greater distance from the brain on the ipsilesional side, which led to interhemispheric differences in SNR. The position of one eye was monitored at $120 \mathrm{~Hz}$ during scanning using the pupil-corneal reflection tracking system (RK-726PCl, Iscan, Cambridge, MA). Prior to each scanning session, a contrast agent, monocrystalline iron oxide nanoparticle (MION or Sinerem®), was injected into the animal's femoral or saphenous vein $(8-11.5 \mathrm{mg} / \mathrm{kg})$. This contrast agent improved the contrast-to-noise ratio and the spatial specificity of the MR signal compared to blood oxygenation level-dependent (BOLD) measurements (Mandeville JB and JJ Marota 1999; Vanduffel W et al. 2001).

Before acquiring functional data in each session, a magnetization-prepared rapid-acquisition gradient echo (MPRAGE) sequence (256 sagittal slices, $256 \times 256$ inplane matrix, repetition time $(T R)=2.2 \mathrm{~s}$, echo time $(T E)=2.07 \mathrm{~ms}, 0.5 \mathrm{~mm}$ isotropic voxels, flip angle $=9^{\circ}$ ) was performed to visualize needle tracks and injection locations of each infusion. 
Data were acquired using a mixed-block design. Each functional time series or run (160 functional volumes, $320 \mathrm{~s}$ ) consisted of four repetitions of three conditions (Fig. 1D): fixation baseline (10 acquisitions), CJ (15 acquisitions), and DET task (15 acquisitions). The functional volumes were gradient-echoplanar images (GE-EPI) covering the whole brain $(\mathrm{TR}=2 \mathrm{~s} ; \mathrm{TE}=19 \mathrm{~ms} ; 40$ horizontal slices with a $84 \times 84$ matrix; $1.25 \mathrm{~mm}$ isotropic voxels).

Two sequences of stimulus presentation were used with the fixation condition always followed by either the CJ or the DET task. The order was kept constant within a given run. The number of runs tested daily depended on the performance levels of the monkey and varied between 12 and 48 (mean of $25 \pm 9$ ).

\section{Reversible inactivation of LIP using Brainsight ${ }^{\mathrm{TM}}$}

The targets for the saline and muscimol injections were determined based on activation sites revealed by functional scans acquired prior to the inactivation experiments (using the contrast: CJ vs. fixation; number of pre-injection runs $=32$ and 37 for $\mathrm{M} 9$ and $\mathrm{M} 10$, respectively) (Fig. 2A). A grid (Crist Instrument) was placed in the recording chamber and subsequent injections were made through the grid holes. The 3-dimensional coordinates of the fMRI-based injection targets were determined relative to the grid using Brainsight software.

\section{Injection procedure}

Prior to each scan session, the monkey was placed in the monkey chair with its head restrained. An Olivier Freeguide ${ }^{\mathrm{TM}}$ articulated arm and double-chuck tool guide were 
fixed to this chair. The needle holder was placed in the tool guide, oriented parallel to the grid and directed along the trajectory of the sulcus to limit damage to neural tissue

when inserting the needle. A solution of $6 \mu \mathrm{g} / \mu \mathrm{l}$ muscimol (Sigma, Aldrich) dissolved in saline was injected using a $5 \mu \mathrm{l}$ Hamilton syringe fitted with a 33 gauge stainless steel needle. We injected along one to three needle tracks and infused muscimol at two to three depths along each track separated by $2 \mathrm{~mm}$ (Fig. 2A). The volume injected at each site was $0.6 \mu \mathrm{l}$ and was delivered slowly in small steps of $0.2 \mu \mathrm{l}$ every 2 minutes to avoid pressure damage. The total volume injected in each experiment ranged from 1 to $5.4 \mu \mathrm{l}$.

Control data, after the injection of an equal amount of physiological saline at the same sites as the muscimol injections, were obtained one to three days before or after each inactivation session. The injections were performed into left LIP of M9 and right LIP of M10 (Fig. 2A).

\section{Data selection}

We acquired 154 runs for M9 of which 77 after muscimol injections for the CJ/DET tasks. For M10 we collected in total 285 runs for the CJ/DET tasks (109 runs after muscimol injections). We analyzed only the volumes acquired after infusions in the correct location (LIP) based upon the anatomical images acquired immediately before the functional scan session. When the anatomical images revealed a needle track in the medial bank of the IPS, the entire session was excluded from further analysis. Note that we did not co-inject an MR contrast agent to visualize the spread of the injection (such as Manganese or Gadolinium as in, e.g., Wilke et al. (2012)) since this would have been 
detrimental to the functional EPIs at the site of injection. Furthermore, a run was selected only if the monkeys had been fixating for more than $87 \%$ of the total run duration (within a $2 \times 3^{\circ}$ window). For the CJ/DET tasks of M9, this resulted in 56 and 36 analyzed runs for the muscimol and saline condition, respectively (Table 1). For M10, 52 (muscimol) and 36 (saline) CJ/DET runs were analyzed (Table 1).

The data selection (fixation $>87 \%$ ) combined with the strict behavioral requirement that monkeys maintain fixation within a $2^{\circ} \times 3^{\circ}$ window, centered on the fixation point, minimized the potential effects of fixation variability on the fMRI data. We analyzed only trials embedded in runs with overall high performance levels. Selecting trials without considering the overall behavior during runs in which they are embedded may bias the results towards trials that were correctly executed only by chance.

\section{Statistical analyses}

Statistical analyses, including behavioral data analyses, were performed using the following software packages: MATLAB and Statistics Toolbox Release 2012b, The MathWorks, Inc., Natick, Massachusetts, United States; SPM5 (http://www.fil.ion.ucl.ac.uk/spm/); Freesurfer (https://surfer.nmr.mgh.harvard.edu/), Caret (http://sumsdb.wustl.edu/); FSL (https://fsl.fmrib.ox.ac.uk/fsl/fslwiki).

Off-line image reconstruction was performed to overcome issues arising from body motion. Details about the image reconstruction protocol have been described in (Kolster $\mathrm{H}$ et al.). Briefly, the raw EPI images were corrected for lowest-order offresonance effects and aligned with respect to the gradient-recalled-echo reference images before performing a SENSE (sensitivity encoding) image reconstruction 
(Pruessmann KP et al. 1999). Corrections for higher-order distortions were performed using a non-rigid slice-by-slice distortion correction.

Time series were further analyzed using SPM5 (Worsley KJ and KJ Friston 1995) (http://www.fil.ion.ucl.ac.uk/spm/) and MATCH software. Spatial preprocessing consisted of realignment and rigid co-registration with a template anatomy (M12; 0.35 $\mathrm{mm}$ isotropic voxels, in stereotaxic space (Ekstrom LB et al. 2008)). To compensate for EPI distortions and inter-individual anatomical differences, functional images were warped to the template using non-rigid matching techniques (BrainMatch). The images were resliced to $1 \mathrm{~mm}$ isotropic voxels and finally smoothed with an isotropic Gaussian kernel $(F W H M=1.5 \mathrm{~mm})$. A mask capturing the full extent of the template brain but excluding the cerebellum, the eyeballs and other non-cerebral tissue was used to specify the voxels for General Linear Model (GLM) estimation. The pre-processed fMRI data were further analyzed in an event-related manner.

\section{Region of interest (ROI) analysis}

Functional ROls (set (a)) were determined based on results of functional scans acquired prior the inactivation experiments (block design analysis, using the contrast: CJ versus fixation). All voxels with a t-score above 4.89 (FWE, $p<0.05)$ were included in the ROI. The borders of visual areas were identified on the flattened cortical representation using retinotopic mapping data previously collected by our research group (Janssens $\mathrm{T}$ et al. 2014; Kolster $\mathrm{H}$ et al. 2014). Parietal areas were determined using the subdivisions of the intraparietal sulcus following (Durand JB et al. 2007; Nelissen K et al. 2011; Caspari $\mathrm{N}$ et al. 2015). Frontal regions (F1, F2, F3, F4, F5a, F5p, F5c, F6, F7, FEF, 45A, 45B, 
$46 \mathrm{v}$ ) were defined on the template brain (M12) using anatomical criteria. Five of these frontal areas (F5a, F5p, F5c, 45B and FEF) were delineated according to (Nelissen $\mathrm{K}$ et al. 2005). Other frontal ROls were determined based on anatomical criteria of (Rizzolatti G and G Luppino 2001). The borders of orbito-frontal regions were defined according to (Ferry AT et al. 2000). The ROI definitions were also compared with (Felleman DJ and DC Van Essen 1991), and complemented with few missing temporal and parietal ROls from (Reveley C et al. 2016).

The main set of ROls (a) were defined separately for each monkey using independent data acquired prior the inactivation experiment. Both the activations and the set of reference ROls were overlaid onto the template anatomy M12. This monkeyspecific ROI set (a) used for the main analyses (as shown in Figs. 6 and 8) included 16 ROIs: V1, V2, V3, V4, V4A, MT, MSTV, OT, PITd, PITv, LIP (pooled LIPa and LIPi (Caspari $\mathrm{N}$ et al. 2015)), F5a, FEF, 46v, 45A, and 45B (yellow labels, Fig 4, Fig 4S0). An additional set of ROls (b) consisted of ROls defined previously in our lab and was used to provide more comprehensive results, independently of the ROls based on previously-acquired CJ activations. Set (b) included 18 additional ROls (used in Fig. $8 S 1$ and Fig. 8S2): V4t, V6, TEm, TEav, TEad, TEpd, TEpv, PGa, TPO, LIPa, LIPi, F1, F2, F3, F5c, F5p, F7, 44 (green labels, Fig 4, Fig 4S0). On the flat maps we show the larger set of ROls (b).

We sampled percent signal change (PSC) within each ROI for each task condition with the MarsBaR ROI toolbox of SPM. Fixation epochs served as baseline. To measure the effects of inactivation on PSC, we compared differences between fMRI signal changes in muscimol and saline sessions. Standard bootstrapping was used to 
evaluate the distribution of the mean and confidence intervals for these differences. The bootstrapping (Efron B and R Tibshirani 1996) consisted of 1000 repetitions, randomly resampling with replacement the two sets of data for injections with saline and injections with muscimol. To construct confidence intervals $(\mathrm{Cl})$, we used bias-corrected and accelerated bootstrapping, adjusting for both bias and skewness in the bootstrap distribution (Efron B 1987; DiCiccio TJ and B Efron 1996; Efron B and R Tibshirani 1996). This bootstrap analysis was performed using MATLAB and Statistics Toolbox Release 2012b (The MathWorks, Inc., Natick, Massachusetts, United States). We used Bonferroni correction to adjust the $\mathrm{Cl}$ for multiple comparisons ( $\mathrm{N}=16$ (number of ROls) x 1000 (number of repetitions for bootstrapping)).

\section{Event-related analysis}

The experiment relied on a mixed event-related/block design. Due to the variability (jittering) in trial duration and behavioral events, we could analyze the data in an eventrelated manner (note that fast event-related designs are perfectly applicable (Burock MA et al. 1998)). Although event-related designs have lower sensitivities compared to block designs, they allowed us to segregate correct from incorrect responses (Chee MW et al. 2003) and to correlate task-related activations with behavioral performance.

It's noteworthy that results of block and event-related analyses were quite robust and reproducible in terms of relative-effect size and spatial location (e.g. spatial correlations between results obtained by the same contrasts evaluated in the two data processing approaches exceeded 0.87). For the sake of simplicity, however, we illustrate our findings using only the event-related analyses. 
Fixed-effect analysis

First, we performed event-related fixed-effect single-subject analyses (FX). The regressors included fixation, $\mathrm{CJ}$ correct trials with left target, $\mathrm{CJ}$ correct trials with right target, CJ correct trials without target, DET correct trials with left target, DET correct trials with right target, DET correct trials without target, all incorrect trials, and all aborted trials. Each trial was modeled with one predictor. Correct trials were modeled separately from incorrect and aborted trials. The small number of incorrect and aborted trials and their heavily uneven distributions over the different conditions, prohibited us from using these types of trials in specific contrasts. For GLM purposes, they were collapsed separately into two unique regressors. Hence, a comparison with correct trials was meaningless. Realignment parameters were included as covariates of no-interest to reduce (brain) motion artifacts.

We calculated the following contrasts (using only correct trials): CJ correct left and right target vs. fixation (CJ task activations); DET correct left and right target vs. fixation (DET task activations); CJ and DET correct left and right target vs. fixation (mixed visual search detection (MVSD) task activations). We compared the experimental conditions ( $\mathrm{CJ}$ and $\mathrm{DET}$ ) to the fixation baseline at a threshold corrected for multiple comparisons.

Second level analysis 
First, we ran a random fixed -effects (RFX) analysis that allowed us to estimate the between-run variability. The details of this first GLM analysis were similar to those described above.

Additional second level analyses were performed by contrasting the contrast images obtained from the RFX analysis. Two sample T-tests were used to compare different conditions: saline vs. muscimol for CJ, DET and MVSD tasks; CJ vs. DET for saline and muscimol tests.

We show maps based on both first-level fixed and second-level random mixed effects GLM contrasts. Minor discrepancies may arise between both types of analysis, as the statistical power is different, resulting in small differences at voxel level.

For both the first and second level analyses, the significance level was set at $p<$ 0.05 and two methods correcting for multiple comparisons were applied independently: FDR corrections $(p<0.05, T>3.1$; performed in Matlab using either SPM, the Statistical Toolbox, or the two-stage (Benjamini $Y$ et al. 2006) procedure) and clusterwise corrections with cluster-wise p-values $<0.05$ (Freesurfer (https://surfer.nmr.mgh.harvard.edu/) (Fischl B 2012)). The two correction approaches showed consistent outcomes, thus strengthening our results. The t-score maps of these contrasts were overlaid onto a template anatomy (M12).

Flattened cortical representations were created with Caret (http://sumsdb.wustl.edu/) (Van Essen DC et al. 2001) and Freesurfer (https://surfer.nmr.mgh.harvard.edu/) (Fischl B 2012).

The difference in the number of runs was compensated by selecting for all analyses an approximately equal number of total trials for both monkeys, and for both 
saline and muscimol conditions (mean \pm SEM: $340 \pm 9$ ). All GLM analyses are based on correctly-executed trials.

\section{Correlation analysis}

One of our goals was to show correlations between the behavior of the subjects and brain activity. Therefore, we calculated Pearson correlations between percent correct responses and the beta values (for each task condition) for each run and for each experimental condition (muscimol and saline injections).

The Pearson correlations were evaluated independently for each voxel in the brain, giving us the ability to represent the results either on the brain volume or on flat maps.

Next, all Pearson correlations, which we will refer to as behavioral correlations, were Fisher r-to-z transformed (ZR). To evaluate the impact of the inactivation, we calculated differences between sessions with muscimol and those with saline injections. Statistical significance for correlations or differences in correlations (r-to-z transformed) was established using T-tests corrected for multiple comparisons (FDR corrections (Fig. 8), $p<0.05$; clusterwise corrections with clusterwise $p$-values $<0.05$ (Fig. 7); FDR correction applied to the data in Fig. 7 showed results consistent with the clusterwise correction).

\section{Linking changes in fMRI activations with changes in behavioral correlations}

Evaluation of the combined effects of the muscimol injections on fMRI activations and on the behavioral correlations offers insights into the behavioral relevance of muscimol- 
induced changes in fMRI activations. Therefore, we directly compared muscimolinduced changes in fMRI activations (dT; Fig. 5) with changes in correlations between behavior and task-driven activity (which we refer to as behavioral correlations; dZR; Fig. 7). Specifically, we performed a conjunction analysis, or logical "and" operation between the maps quantifying changes in fMRI activations (Fig. 5; dT-maps) and those quantifying changes in behavioral correlations (Fig. 7; dZR-maps). Surface-based analyses using Freesurfer were performed (https://surfer.nmr.mgh.harvard.edu/).

Previous analyses showed that values $(d T$ and $d Z R) \geq 3.1$ are significant $(p \leq$ 0.05) after FDR or clusterwise corrections for multiple comparisons. We started the conjunction analysis using a more generous threshold of $(d T$ and $d Z R) \geq 1.64(p=0.05$, uncorrected for multiple comparisons) and we independently analyzed all possible pairs of combinations (dT and dZR): (a) increase-increase (dT > 1.64 and dZR > 1.64); (b) decrease-decrease ( $\mathrm{dT}<-1.64$ and dZR $<-1.64$ ); (c) decrease-increase (dT $<-1.64$ and $d Z R>1.64) ;(d)$ increase-decrease $(d T>1.64$ and $d Z R<-1.64)$.

All differences ( $d T$ and $d Z R$ ) were calculated between muscimol and saline conditions. The initial conjunction analysis gave us 4 different sets of indices corresponding to conjunction-vertices on the flat maps that were used to generate four pairs ( $d T$ and $d Z R$ ) of conjunction maps. Next, we retrieved from each conjunction map the maximum values for each independently-defined ROI. Values greater than 3.1 were considered significant ( $p \leq 0.05$, after both FDR and cluster-wise corrections for multiple comparisons). 
Fig. 8 shows the results for ROls (a) whereby the conjunction analysis revealed increases for both dT and dZR (Fig. 8A and C: increases in activation; Fig. 8B and D: increases in correlation).

We independently analyzed all possible pairs of combinations of changes in fMRI and change in correlation with behavior: increase-increase, decrease-decrease, decrease-increase, and increase-decrease. Since the conjunction analysis originated from unsmoothed maps and because we retrieved the maximum values for each independently-defined ROI, some minor differences can be discerned between Fig. 8 and Fig. 7 (e.g., FEF for the ipsilesional hemisphere of M10).

\section{Results}

We injected muscimol, a GABAA agonist, at 21 sites (11 in the left hemisphere of M9 and 10 in the right hemisphere of M10, Fig. 2A) distributed throughout LIP in two monkeys performing two different cognitive tasks. Control data were obtained 1 to 3 days before or after each injection of muscimol. On these days, the same quantity of saline was injected at the same locations. We analyzed the behavioral and functional data obtained after injections in LIP and for all runs during which the animals fixated at least $87 \%$ of the run within a $2 \times 3$ degree fixation window (see Methods).

\section{Behavioral effect of LIP inactivation}

Target detection accuracy

The monkeys were trained on a conjunction search task in which they had to detect a bar target amongst competing distracters based on color and orientation (CJ task). In the detection task (DET), they had to detect the brightening of part of the background 
while the same, but now task-irrelevant, bars were presented as during the CJ task. In all tasks, the monkeys indicated the presence of the targets (or brightening events) with a right hand movement (see Fig. 1).

We calculated both d-prime and its non-parametric version, a-prime (Zhang $\mathrm{J}$ and ST Mueller 2005). The results are presented in Figures 2B (CJ) and Fig. 2S1 A (DET), and the corresponding statistics are summarized in Table 2 and 3 . KruskalWallis [factors: (f1) M9 vs. M10, (f2) saline vs. muscimol, (f3) CJ vs. DET, (f4) ipsilesional vs. contralesional, and (f5) set size] showed significant effects on d-prime/aprime for f1, f2, f4 and f5. Most importantly, both d-prime/a-prime indicated differences between saline vs. muscimol (f2) for both tasks and both monkeys. This salinemuscimol difference proved significant in both the CJ and the DET tasks (f3, Table 2). Moreover, d-prime/a-prime indicated significant differences between ipsilesional vs. contralesional targets (f4), both for CJ and DET (Table 2, M10). Hence, there was a contralesional bias (contra>ipsi) in M10, but not M9. We suggest two factors that could contribute to this bilateral deficit. First, the unpredictable location of the target requires equal monitoring of both hemifields, possibly leading to an equal attention being given to both hemifields, explaining the deficit for targets in the ipsilesional hemifield. Accordingly, given the extreme overtraining prior to scanning (> $2.510^{6}$ trials in total), our monkeys may solve the conjunction search in a more parallel manner. Although speculative, this interpretation is corroborated by a very weak behavioral set size effect (only for set size 2 vs. 8; Table 2, Kruskal Wallis test corrected for multiple comparisons). Second, the deployment of attention relies on balanced inter-hemispheric 
interactions (Balan PF et al. 2017), and any perturbation of this equilibrium can affect the distribution of attention across the two hemifields.

Figure $2 \mathrm{~S} 2 \mathrm{~A}$ also shows the target detection accuracy of the two monkeys during the $\mathrm{CJ}$ task for targets presented in the contra- and ipsilesional hemifield, which corroborates the d-prime/a-prime analyses. As expected from covert conjunction search studies (Wardak C et al. 2004), target detection accuracy for contralesional targets declined after LIP inactivation in both monkeys (Table 3S). A clear deficit was also observed for targets presented in the ipsilesional hemifield (Table 3S).

Response-accuracy analysis for the DET task showed similar results (Fig. 2S2 B). Kruskal-Wallis tests [factors: (f1) M9 vs. M10, (f2) saline vs. muscimol, (f3) CJ vs. DET, (f4) ipsilesional vs. contralesional, and (f5) set size] showed significant effects on response accuracy (Table 2S; Fig. 2S2): f2, f4 and f5 for correct responses, all factors for the incorrect responses, and f1 and f2 for aborted trials. Most importantly, the percent correct responses differed between saline vs. muscimol (f2) for both tasks, and the $\mathrm{CJ}$ and DET tasks show similar results (Table $2 \mathrm{~S}$ and $3 \mathrm{~S}$ ).

Percent correct responses differed for ipsilesional vs. contralesional targets (f4) for both tasks (Table 2S). Although a muscimol-induced deficit for both contra- and ipsilesional targets is observed in almost all cases, the inactivation is more effective for the contralesional targets (Fig. 2S2; Table 2S and 3S). Furthermore, in all cases (except the DET task for M10), performances after the inactivation exceeded chance level (Fig. 2S2).

Finally, a significant set-size effect was observed only for the extreme set sizes (set size 2 vs. 8; Table 2S, Kruskal-Wallis test corrected for multiple comparisons). 
In line with the behavioral results presented in figure $2 \mathrm{~S} 2$, within the selected runs (surviving the $>87 \%$ fixation criterion), there is a tendency for increased fixation breaks after LIP inactivation (M9; Kruskal Wallis $X^{2}(1,502)=16.9, p=410^{-5}$; with no significant effect for M10 $\left.\left(x^{2}(1,496)=1.7, p=210^{-1}\right)\right)$.

\section{Reaction times}

First, it is important to note that we did not use explicit reaction-time tasks since the monkeys had ample time to respond. Yet, as shown in Fig. 2C (CJ) and in Fig. 2S1 B (DET), reaction times differed between saline and muscimol trials for both monkeys, with no significant differences between ipsi- and contra-lesional targets (Table 4S).

For M9, injections of muscimol clearly increased reaction times for detecting the contra- and ipsilesional targets for all set sizes and for both the CJ and DET tasks (Table 4S; Fig. 2C and Fig. 2S1 B). Reaction times of M10 increased after muscimol administration only for CJ (ipsilesional targets; Fig. 2C) and DET (contralesional; Fig. 2S1 B). This could be due to the generally higher reaction times of M10 (ceiling effect). Unlike M9, M10 showed different reaction times for the CJ and DET tasks. Finally, a reaction time set-size effect was stronger for M9, likely because of a ceiling effect in M10.

\section{CJ and DET tasks yielded similar behavioral and fMRI activations effects}

It needs to be noted that the CJ and DET tasks were very similar (both in terms of difficulty and visual stimulation) and that within a run we mixed CJ and DET trials (Fig. 1D). Therefore, instead of two distinct tasks we consider this as a single task. Most 
likely, the monkeys solved both tasks using similar strategies which may have been triggered by the extreme overtraining of the monkeys prior to scanning $\left(>2.510^{6}\right.$ trials in total). Moreover, the displays were identical for both tasks which were continuously interleaved. Although speculative, our monkeys may solve the conjunction search, which is supposed to be a serial task, in a parallel manner (Sireteanu $R$ and $R$ Rettenbach 1995; Leonards U et al. 1998; Sireteanu R and R Rettenbach 2000; Wardak C et al. 2012), as corroborated by the unexpectedly small set-size effect. Consistent with these similarities, both CJ and DET tasks yielded highly similar behavioral effects (see "Target detection accuracy").

This raises the possibility that the networks activated by CJ and DET, and the muscimol-induced effects in these networks, are also similar. To address this, we used a second-level GLM analysis, followed by a two-sample T-test comparing CJ (contrast CJ vs. fixation) vs. DET (contrast DET vs. fixation) for both saline and muscimol injections (for each monkey). The resulting volumes showed no significant differences (similar results were obtained with an FDR-correction $(T \geq 3.1)$ and a cluster-wise correction for multiple comparisons with $p$-values $<0.05$ ) for all tests.

To further support this conjecture, we show activations separately for the $\mathrm{CJ}$ and DET tasks, and the 2-sample T-tests directly comparing activations of both tasks for saline (Fig. 4S1 for M9, and Fig. 4S3 for M10) and muscimol (Fig. 4S2 for M9, and Fig. 4S4 for M10) conditions. All corresponding pairs of flat maps for CJ and DET tasks were very similar. The Pearson correlation between maps ranged between 0.72076 and $0.87013, p=0$ ) with similar activation strengths (slope of the regression DET $=f(C J)$ ranged from 0.84847 to $1.21833, p=0, R^{2}$ ranged from 0.5195 to 0.7571 ). 
Furthermore, the muscimol-induced effects on these networks are also indistinguishable ( $T \geq 3.1$, FDR and clusterwise corrections for multiple comparisons with $p$-values $<0.05$ showed no significant differences in either monkey). Supplementary figure $5 \mathrm{~S} 1$ shows the contrasts muscimol vs. saline separately for CJ and DET tasks in each monkey. All corresponding pairs of flat maps for CJ and DET task were similar. Their correlation ranged from 0.48263 to $0.64252, p=0$ ) and the slope of the regression DET $=f(C J)$ ranged from 0.48926 to $0.67905\left(p=0, R^{2}\right.$ ranging from 0.2329 to 0.4128$)$.

Hence, both the behavioral and fMRI activations support our post-hoc assumption that $\mathrm{CJ}$ and DET were similar tasks solved using similar strategies by the monkeys. In fact, this result was unintentional and unexpected, but we turned it to our advantage by analyzing $\mathrm{CJ}$ and DET trials together for the remainder of the result section, thereby increasing the statistical power of our event-related fMRI analyses. Accordingly, the CJ and DET trials taken together will be designated the mixed visual search detection (MVSD) task from here on. Since we noticed only weak set-size effects on fMRI activity (only in M10) and on performance (only for set size 2 vs. 8), and since studying the well-known set size effect is not the goal of the study, we also decided to average data across set sizes.

\section{Reaction time variability does not influence the fMRI activations}

To examine the potential contribution of reaction time (RT) differences on our activations, we added an RT regressor in our GLM. Comparisons (two-sample T tests) between GLM random-fixed effect (RFX) results with and without the RT regressor 
show that they are indistinguishable. This is illustrated in flat maps for GLM RFX analysis with (panels A) or without (panels B) the RT regressor, and two-sample $\mathrm{T}$ test comparisons between them. Panels in C illustrate left and right hemispheres, shown for saline (Fig. 4S6 for M9, and Fig. 4S8 for M10) and muscimol conditions (Fig. 4S7 for M9, and Fig. 4S9 for M10). All corresponding pairs of flat maps with vs. without RT regressor (i.e., each row of panels A vs. panels B in Fig. 4S6 - 4S9) were very similar. Their correlations ranged from 0.99896 to $0.99979, p=0$ ), and the activation strengths were similar (slopes of the regression ranged from 0.99842 to $1.01097, p=0, R^{2}$ ranged from 0.9979 to 0.9996$)$.

In addition, we show the contrasts muscimol vs. saline for GLM RFX with (panels A) and without (panels B) RT regressor (Fig. $5 \mathrm{~S} 2$ for M9, and Fig. $5 \mathrm{S3}$ for M10). The correlations between these flat maps with and without the RT regressor ranged from 0.99835 to $0.99870(p=0)$ with regression slopes ranging from 0.99197 to $1.00285(p=$ $0, R^{2}$ ranged from 0.9968 to 0.9974$)$.

In conclusion, there is no evidence supporting the idea that muscimol-induced increases in RTs can explain our observed results.

\section{Effect of LIP inactivation on local fMRI activity}

A GABA agonist reduces local neuronal activity (e.g. (Andrews PR and GA Johnston 1979). Additionally, it has been shown that inhibition of GABA transaminase causes increased GABA levels and decreased fMRI signals in anesthetized rats (Chen $Z$ et al. 2005). Therefore, we expected reduced fMRI activity at the site of injection after infusion of muscimol. As shown in figure $3 \mathrm{~A}$, LIP was significantly activated during the MVSD 
task after saline injections into LIP. Existing asymmetries between the ipsi- and the contralesional hemispheres may be related to potential microlesions caused by the frequent injections, but is more likely due to a difference in temporal fMRI signal-tonoise ratios due to the asymmetric position of the coils (see fMRI scanning). Nevertheless, infusion of the same quantity of muscimol into LIP significantly reduced local fMRI activity relative to saline injections (Fig. 3B and inset: Kruskal-Wallis, $p<$ 0.05). We conclude that injection of a GABA agonist effectively decreased local taskdriven fMRI activity. In addition to the neuro-metabolic effect, muscimol also exerts a local vasodilator effect on brain parenchyma (Fergus A and KS Lee 1997) which should increase fMRI signals. Therefore, it is reasonable to assume that the observed decrease in local fMRI activity in the present experiment underestimated the actual neuro-metabolic effect of muscimol.

Additionally, after muscimol injections, we found patches in the LIP ROI (yellow, Fig. $3 \mathrm{C}$ and E) showing decreased task-driven fMRI activations in the injected hemisphere (blue voxels) while similar voxels in the opposite hemisphere show increased (red voxels) fMRI activity (Fig. $3 \mathrm{C}$ and $3 E)(p<0.05$, FDR corrected). Of note is the striking inter-hemispheric homotopic correspondence between the two patches within the IPS showing opposite inactivation effects. The difference in percent signal change (muscimol vs. saline) for the fMRI activity in these voxels is shown in figures 3D and $3 \mathrm{~F}$ (mean $\pm \mathrm{Cl} / 2$ at $\mathrm{p}=3 \mathrm{10}^{-5}$ ). Thus, both spatially and quantitatively we found a significant difference between the effects of saline and muscimol injections in LIP.

\section{Effect of reversible LIP inactivation on the MVSD task network}


Small intracortical injections of muscimol decreased fMRI activity at the site of injection. This focal reduction in activity propagates through the network of areas functionally connected with this site. Theoretically, this should cause a reduction in fMRI activity throughout the entire functional network since most cortico-cortical connections are excitatory (Salin PA and J Bullier 1995). Alternatively, other nodes of the existing network may compensate for the loss of activity in LIP by increasing their own activity levels and/or by recruiting additional nodes into the network that were previously not activated. To test for these alternative hypotheses, we investigated the functional consequences of a reversible LIP inactivation throughout the cortex.

The network of cortical areas activated during the MVSD condition (MVSD fixation, referred to as 'MVSD-related' activity) is shown on the flattened representations of the ipsi- and contralesional hemispheres of both monkeys (Fig. 4A, 4B and Fig. 4S0). The regions activated during MVSD after saline injections in LIP are indicated by the blue outlines (Fig. 4) or by a red-yellow color scale in figure $4 \mathrm{~S} 0$. The red-yellow colorcode in figure 4 represents MVSD-related activations scanned after inactivation (muscimol) of LIP. Except for a relatively small increase in the sizes of the activated areas after the muscimol injections, especially in early visual cortex, no dramatic shifts in the topography of the MVSD network can be observed. A second-level analysis (Fig. 5), comparing MVSD-related activity between muscimol and saline conditions, shows heterogeneous changes in activations across the network, but qualitatively more increased (Fig. 5; contrast muscimol - saline) than decreased (Fig. 5S0; contrast saline - muscimol) muscimol-driven changes in fMRI activity. In both monkeys, MVSD-driven activity increased after muscimol injections (colored red-yellow) in subsectors of visual, 
parietal and frontal areas of both hemispheres. In M10, injections of muscimol resulted in lower fMRI activity (compared to saline; Fig. 5S0)) in subsectors of prefrontal, visual and parietal areas, which could not be observed in M9. A detailed list of areas affected by muscimol is described below.

\section{Effect of muscimol on fixation-related fMRI.}

Complementary to the stringent selection criteria for including runs in the statistical analyses (fixation $>87 \%$ ) in order to limit fMRI variability induced by unequal fixation behavior, we also tested whether muscimol injections in LIP altered fixation-related fMRI activity. Specifically, we compared beta strengths for fixation trials between the muscimol and saline conditions using a two sample T-test, corrected for multiple comparisons (FDR $p=0.05, T>4.1$, and clusterwise corrections gave similar results). Results showed no significant differences between the fixation-related activations during the muscimol and saline conditions for either monkey (Fig. 4S5).

\section{LIP inactivation alters the strength of task-related activity}

We further quantified the muscimol-driven changes in fMRI activity using more sensitive $\mathrm{ROI}$ analyses. We first defined ROIs based on the results of independent functional scans acquired prior to the inactivation experiments (contrast $\mathrm{CJ}$ vs. fixation) and then compared the percent fMRI signal changes between the saline and muscimol conditions in these ROIs (Fig. 6; see Region-of-interest analysis).

The ROI analysis is in line with our second hypothesis: injection of muscimol mainly increased MVSD-related activity (mean $\pm \mathrm{Cl} / 2$ at $\mathrm{p}=210^{-6}$ ). In general, more 
ROls (39 across both animals) showed significantly higher, instead of lower (20 ROls), MVSD-related activity after muscimol injections compared to saline sessions (Fig. 6). This effect was even more pronounced in the ipsilesional hemisphere: 23 ROls showed significantly increased activity while only 7 areas revealed a decrease in activity (Fig. 6A and B, left panels). More specifically, increased muscimol-induced activity was observed in ipsilesional visual areas V1, V2, V3, V4, V4A, MSTV, PITd and PITv, in premotor area F5a, (pre)frontal areas FEF and 45A (Fig. 6A, left), and in contralesional V1, V3, MT, MSTV, PITd and PITv of M9 (Fig. 6A, right). In the same animal, we found lower fMRI activity after muscimol injections in ipsilesional MT, 45B and 46v and in contralesional V2, V4 and V4A and the (prefrontal) areas FEF, 45A, 45B and 46v. For M10, we found higher MVSD-related activity in ipsilesional visual areas V4, V4A, MT, MSTv, PITd, PITv, F5a, FEF, 45B and 46v (Fig. 6B, left), and contralesional visual areas V4A, PITd and (pre)frontal areas FEF, 45A, 45B and 46v (Fig. 6B, right). Lower activity after muscimol injections were found only in ipsilesional area 45A and contralesional V2, V3, V4, MSTV, PITV and F5a of M10. Thus, areas showing a consistently higher muscimol-induced activity across subjects were ipsilesional areas V3, V4, V4A, MSTv, PITd, PITv, F5a, FEF (Fig. 6A and B, left) and contralesional PITd (Fig. 6A and $\mathrm{B}$, right), with the changes being most pronounced and consistent in ipsilesional FEF. In contrast, lower activity after muscimol injections was observed only in ipsilesional OT and in contralesional V2 and V4 of both monkeys.

Although we observed a clear reduction in fMRI activity at local injection sites within LIP of both animals (Fig. 3), it should be noted that activity in the remainder of LIP 
may increase (see e.g. M9, Fig. 6A). The latter effect was not consistent across monkeys, however.

\section{Correlation between activity and behavior}

If changes in MR activity induced by muscimol reflect compensatory rather than passive mechanisms, one predicts that the correlation between the strength of MR activity and performance levels should significantly change after muscimol injections. We therefore tested for changes in correlation between task-driven fMRI activity and the percent correct target detection induced by the muscimol injections. To this end, we first calculated the correlations between average percent correct per run and average betas per run for the MVSD task for each voxel. After r-to-z Fisher transformation, we evaluated the differences between muscimol and saline conditions. Figure 7 shows that inactivation of LIP significantly increased the correlation between performance (percent correct trials) and fMRI activity in several areas outside LIP (Fisher r-to-z, both FDR and cluster-wise corrections with $p$-values $<0.05$ gave similar results). Examples of areas in which the correlations during MVSD significantly increased after LIP inactivation include V1, V2, V4A, OT, TPO, F5p, F5c, FEF, 12 and insular cortex (Fig. 7). Supplementary figures Fig. 7S1 (M9) and Fig. 7S2 (M10) show flat maps with correlations between behavioral performance and task-based fMRI activity independently for saline and muscimol conditions, and changes in correlation between fMRI activity and behavioral performance for saline and muscimol conditions separately, and for the difference between them. In general, we noticed only very few patches with negative changes in activity-behavior correlation that were not consistent across monkeys. The overall 
increase in such correlation is qualitatively more pronounced in the ipsilesional hemisphere, especially in M10 (Fig. 7, 7S1-2). This result is consistent with the suggestion that the early recruitment of contralesional areas supports recovery by modulating the preserved ipsilesional network (Umarova RM et al. 2016).

It is noteworthy that the increase in the correlation between the performance and beta-values does not necessarily imply that the activity itself increased after the inactivation (e.g., a stronger decrease of activity could be correlated with a stronger behavioral impairment). Accordingly, Figure 7, showing an increase in correlation for the ipsilesional LIP (M9), would suggest that a local mechanism remains important even in the presence of remote compensation. On the other hand, Figure 7S1 (M9) and 7S2 (M10) reveal no strong correlations between the performance and the activity in the inactivated LIP sites. This lack of correlation suggests that post-lesion performance is mainly determined by remote (compensatory) mechanisms rather than the degree of suppression of local activity.

To quantify the correlation results, we performed a conjunction analysis between the correlations obtained in saline and muscimol runs, independently for each monkey, and after applying two different thresholds (Tables $5 \mathrm{~S}$ and $6 \mathrm{~S}$ ). Voxels showing significant correlations are either common to saline and muscimol (conjunction), or belong to only one of them (disjunction). It could be argued that the first type of voxels (conjunction) retain signals critical to performing the task, hence are also involved in compensatory mechanisms (although no muscimol-induced changes are observed). However, even at lower threshold (Table 5S), we already noticed a clear dominance of disjunction over conjunction voxels. When FDR correction was imposed (Table 6S), 
there are virtually no conjunction voxels remaining. Hence, this data strongly suggests that those voxels exhibiting changed correlations between activity and behavior contribute most to compensatory mechanisms.

\section{Connecting LIP-inactivation-induced increases in fMRI activation with increases in correlation between behavior and task-driven activity.}

So far, we have focused separately on muscimol-induced changes in fMRI activity (dT;

Fig. 5 and Fig. 6) and on changes in correlations between behavior and activity (dZR;

Fig. 7) (note that these two types of changes can happen in different voxels). To make inferences concerning the behavioral relevance of the altered fMRI activity, we also tested which of the voxels showing altered fMRI activity (dT; Fig. 5) exhibited significant differences in behavioral correlations (dZR; Fig. 7) after muscimol injections in LIP and vice versa, using a conjunction analysis.

We separately analyzed all possible pairs of change in fMRI and changes in correlation with behavior: increase-increase, decrease-decrease, decrease-increase, and increase-decrease. Interestingly, the last three combinations revealed only one ROI showing a common trend in both monkeys: area F4 (dT = -2.66 and dZR = -3.03 in M9; $\mathrm{dT}=-2.58$ and $\mathrm{dZR}=-2.68$ in M10). Therefore, we show only the ROls showing increases in fMRI and increased behavioral correlations (Fig. 8, Fig. 8S1 for an extended set of ROls (set b)). In Fig. $8 S 2$ we show fMRI activity and behavioral correlation for muscimol and for saline for the extended number of ROls (set $b$ ). Figure 8 shows the maximum $t$ - and Z-score values for sub-regions of pre-defined ROls that revealed muscimol-induced increases in both fMRI activations and behavioral 
correlations. Fig. 8A (ipsilesional) and C (contralesional) represent t-score values of differences in fMRI activity (dT; muscimol - saline) $\geq 2.5$ corresponding to changes in behavioral correlations (dZR; muscimol - saline) $\geq 2.5$ for each ROI. Similarly, Fig. 8B (ipsilesional) and $\mathrm{D}$ (contralesional) represent Z-score-values of differences in the behavioral correlations (dZR) $\geq 2.5$ corresponding to changes in the fMRI activations (dT) $\geq 2.5$ for each ROI. All values above the horizontal green line (dT $\geq 3.1$ or $d Z R \geq$ 3.1; filled triangles for M10, or filled circles for M9) are significant at corrected level $(p=$ 0.05, FDR corrected for multiple comparisons). Values below (unfilled symbols) are significant at $p \leq 0.005$ uncorrected for multiple comparisons. ROls that show common trends in both monkeys are marked with vertical lines (continuous line, magenta, at corrected level for both monkeys; dashed lines, light green, only for one subject, and dark green for none of the subjects). The color of the ROI labels follows the same convention.

Regions that showed significant ipsilesional effects in only one of the two types of analyses (in both monkeys and FDR corrected for multiple comparisons) included areas V4, FEF, and 45B (Fig 8A and B). The effects are especially pronounced in FEF, as this is the only area that reached significance (FDR corrected for multiple comparisons) for both tests in both animals.

\section{Differences between the two monkeys}

Slight differences in muscimol-induced behavioral and functional effects were found between the two subjects, with the most pronounced effects seen in M9. This may be related to the exact location of the inactivation in LIP or differences in strategy used to 
solve the task. Alternatively, it may be related to the injected hemisphere (left for M9, right for M10) relative to the dominant hand used during the operant task (right for both monkeys). Nevertheless, we would like to emphasize the consistent and robust effects found in both monkeys, namely 1) the reduced performance levels after LIP inactivation. This was accompanied by 2) higher fMRI activity linked with 3 ) a higher behavioral correlation in the attention network during correctly executed trials of the muscimol vs. saline sessions, particularly in the FEF.

\section{Discussion}

As expected from earlier studies, reversible inactivation of LIP through infusions of small quantities of a GABA-agonist caused a behavioral deficit during a covert conjunction search task (Wardak C et al. 2004). A concurrent reduction of local task-driven fMRI activity was observed after LIP inactivation. Mainly increased task-driven fMRI activity was measured in several up- and downstream areas, and were particularly pronounced in FEF and to a lesser degree in areas V4 and V4A. Importantly, most of the observed increased activity in remote areas is not correlated with the decreased activity in LIP, suggesting that the changes are triggered from areas within the intact functional task network and not from LIP directly. Finally, in some areas remote from the injection the increase in fMRI activity was correlated with behavioral performance levels indicating rapid adaptive - rather than maladaptive - compensatory functional changes in the search task network. The area showing the most pronounced and consistent differences in activity, which are correlated with changes in behavioral performance, was the FEF. Although alternative maladaptive interpretations of these results cannot be entirely 
excluded, a compensatory effect provides the most parsimonious explanation of the results.

\section{Behavioral deficit}

The deficit in performance on the contralesional hemifield during the covert conjunction search confirmed previous work utilizing search tasks (Wardak C et al. 2002, 2004; Balan PF and J Gottlieb 2009). We also observed significantly reduced target detection accuracies for targets presented in the ipsilesional hemifield. This unexpected result may indicate that the ipsilesional saliency maps were also affected. Such an effect may be attributed to those LIP neurons with representations lying up to 20 degrees inside the ipsilateral hemifield (Dunn CA and CL Colby 2010; Kagan I et al. 2010). A mutually nonexclusive, but rather speculative, explanation might be that due to overtraining of our animals, who performed $>2.510^{6}$ trials and always had to detect the same target, the nature of the search task changed from serial to parallel. This is in agreement with previous human and monkey studies showing that subjects can solve a conjunction search task using parallel processes after repetitive training (Nakayama $\mathrm{K}$ and $\mathrm{GH}$ Silverman 1986; Sireteanu R and R Rettenbach 1995; Buracas GT and TD Albright 1999). We may speculate that very narrow saliency peaks are required in the LIP saliency map only when attention has to shift sequentially to different stimuli and not in tasks that can be solved in parallel. Therefore, it is tempting to suggest that in the present experiment the search task did not utilize sharply peaked saliency maps but

instead more broadly tuned maps that extend into the ipsilateral hemifield. This is in accordance with (Falkner AL et al. 2010), who suggested that modulation of the 
inhibitory interactions between neurons in LIP may modulate the sharpness of the saliency peaks. Furthermore, it has been suggested that attention deployment relies on a balanced competition between left and right hemisphere (Balan PF et al. 2017), including left and right LIP. Therefore, the muscimol-induced imbalance may impair attention deployment for both visual hemifields.

\section{Effect of reversible LIP inactivation on remote fMRI activity}

Early stroke studies using cerebral blood flow and BOLD measurements mainly showed increased task-related activations in spared tissue of patients relative to control subjects (Weiller C et al. 1992; Cramer SC et al. 1997; Seitz RJ et al. 1998; Cramer SC et al. 2001). A well-designed stroke study, however, showed a negative correlation between the degree of recovery and the degree of task-related BOLD activations in patients with relatively large lesions but a spared M1 (Ward NS et al. 2003). Here, we correlated the degree of task-driven activity with performance levels of the same subjects before and immediately after induction of a temporary lesion restricted to area LIP. Based on Ward's study and because earlier electrophysiological (Alonso JM et al. 1993; Hupe JM et al. 1998; Ponce CR et al. 2008), and metabolic animal studies (Vanduffel W et al. 1997; Rushmore RJ et al. 2005) mainly described lesion-induced reductions of neuronal or metabolic activity in remote sites, it can be hypothesized that decreased fMRI activity at the site of inactivation would also propagate to regions functionally connected with it.

We found that while activity in area LIP decreased, task-driven fMRI activity in several up- and downstream areas relative to LIP actually increased, particularly in FEF 
and V4. Notably, both regions are heavily implicated in selective attention processes (Corbetta M 1998; Corbetta M et al. 1998; Wardak C et al. 2010; Anderson JC et al. 2011; Simpson GV et al. 2011; Wardak C et al. 2011; Ibos G et al. 2013). In some of the remote areas showing lesion-induced altered fMRI activity, we observed a significant difference in correlation between the degree of fMRI activation and task performance levels (Fig. 7 and Fig. 8). This result suggests that fast compensatory mechanisms are triggered within the functional network after the loss of activity in LIP. Ipsilesional FEF which is tightly coupled with LIP at both the anatomical (Schall J et al. 1995; Stanton GB et al. 1995; Anderson JC et al. 2011) and functional levels (Ekstrom LB et al. 2008; Premereur E et al. 2012; Ibos $\mathrm{G}$ et al. 2013) - is the only region that showed increased fMRI activity and increased correlations with behavior in both animals and at corrected level (Fig. 8). Therefore, it is tempting to speculate that this area may be the main driver for the compensation. Of particular note is area V4 which also shows increased behavioral correlations in both animals (Fig. 8B). This area is activated by search tasks (Ogawa T and H Komatsu 2004; Bichot NP et al. 2005; Wardak C et al. 2010) and its activity can be modulated by the FEF (Moore T and KM Armstrong 2003).

A possible mechanism driving the inactivation effects might be the unmasking of existing long-range excitatory connections in the remainder of the network (e.g. in area FEF), which are normally inhibited by feed-forward inhibition (Jacobs KM and JP Donoghue 1991; Shimizu T et al. 2002; Butefisch CM et al. 2003). Such a mechanism would result in increased activity, exactly as observed in the present experiment. Since such excitatory connections are known to exist between FEF and LIP (Anderson JC et 
al. 2011) a main candidate for initiating these observed compensatory effects is FEF, exactly as we observed.

\section{Comparison with Wilke et al}

In another LIP inactivation study (Wilke M et al. 2012), a memory-guided saccade task was used in monkeys to investigate the effect of unilateral LIP inactivations on brainwide fMRI activity. In one task, the monkeys were required to make a saccade to an instructed unilateral target location. In the second task, they could, again after a memory delay, freely select one of two simultaneously presented targets. During the free choice dual-target trials, Wilke et al. (2012) also observed increased fMRI activity in several frontal and parieto-temporal areas after inactivation of LIP when the monkeys chose the contralesional target. Target competition and selection mechanisms spanning the entire visual field are common aspects between our task and the latter task of the Wilke study - which explains the corresponding outcome (i.e. increased LIP-inactivation induced activity within the task network).

\section{Comparison with patient studies}

The majority of patient studies showing increased activity in sites remote from the lesion, even in the undamaged hemisphere, receive strong support from our study (Chollet F et al. 1991; Weiller C et al. 1992; Seitz RJ et al. 1998; Cramer SC et al. 2001). We observed in several ipsi- and contralesional regions positive correlations between performance levels and fMRI activity. This adds to the evidence that, at least 
immediately after the lesion, functional changes in the latter areas reflect an adaptive recovery mechanism as opposed to a mere epiphenomenon (Johansen-Berg $\mathrm{H}$ 2007).

Other studies reported negative correlations between patient outcome and brain activity observed (e.g. (Ward NS et al. 2003)). This effect might be the result of overcompensation in less-recovered compared to well-recovered patients. Therefore, the authors suggested that this might have led to stronger activations in the spared cortical regions of the functional network, giving rise to the negative correlations in that study.

Results of the present study corroborate those from earlier extinction rather than neglect studies. Indeed, compared to control patients, neglect has been characterized by reduced activation in the ipsilesional parietal and lateral occipital cortex, as well as contralesional FEF (Umarova RM et al. 2011). In contrast, the activation pattern in patients with extinction (regarded as either a 'mild' form of neglect or a distinct syndrome) was represented by an increased activation of the contralesional prefrontal cortex (Becker E and HO Karnath 2007; Vossel S et al. 2011). In both patients with neglect and those with extinction, detection of targets in the contralesional hemifield correlated with an activation in the contralesional prefrontal and parietal cortex (Umarova RM et al. 2011). Our study adds interesting insights to neglect/extinction mechanisms and recovery. The overall increase in activation dominated by the contralesional hemisphere, it should be pointed out, is consistent with the aforementioned human studies.

Our results also speak to the few models attempting to shed light upon the mechanisms explaining (recovery from) neglect (Corbetta M and GL Shulman 2011; Karnath HO and 
C Rorden 2012; Azouvi P et al. 2017). Common to all, the recovery relies on rebalanced activity within a distributed bilateral neuronal network, and the behavioral deficits depend on both local and distal structural and physiological changes (Corbetta $\mathrm{M}$ et al. 2005). On the other hand, studies in spatial neglect and extinction patients arguing for the beneficial involvement of activity in the contralesional hemisphere on the recovery (Thimm M et al. 2009; Umarova RM et al. 2011; Saj A et al. 2013; Umarova RM et al. 2016) are in contrast with the study of Corbetta et al. (2005) arguing for the maladaptive role of the opposite hemisphere. Our findings are not entirely consistent with the interhemispheric rivalry theory suggesting that the dynamic balance between two hemispheres plays an important role in orienting a specific process towards the contralateral hemifield (Kinsbourne M 1977; Cazzoli D et al. 2009). According to the inter-hemispheric rivalry theory, activity increases in the intact, contralesional hemisphere should be maladaptive creating additional imbalances between hemifield representations, amplifying the detrimental effect of the lesion. Since we show bihemispheric activity enhancement associated with correctly performed trials after inactivation, we argue for a compensatory role of the intact hemisphere, similar to findings of Wilke and colleagues who also failed to find strong support for the Kinsbourne hypothesis (Wilke M et al. 2012).

It should further be pointed out that in patient studies, relatively long-term compensatory effects induced by a permanent lesion are measured, in contrast to the immediate effects examined in the current study - which may be different (Corbetta M et al. 2005). Other major dissimilarities with the patient studies are the reversible nature 
of the inactivation, the species, the size of the lesions and how comparisons are made: either across or within subjects.

\section{Conclusion}

A small reversible LIP lesion resulted in a behavioral deficit for a visual search and detection task and altered network-wide task-driven activity. We suggest that the observed rapid increases in activity, particularly in the FEF which is anatomically and functionally closely linked with LIP, partially compensate for the loss of activity in an important node of the search network. We speculate that this compensation is at least to some degree caused by unmasking excitatory long-range connections (known to exist between LIP and FEF) across the functional network.

Funding: This work was supported by Inter University Attraction Pole 7/11; Programme Financing PFV/10/008 and C14/17/109; Hercules II funds; Fonds Wetenschappelijk Onderzoek-Vlaanderen G083111.10, G0A5613, G.062208.10, G0B8617, and Odysseus G0007.12; This project also has received funding from the European Union's Horizon 2020 Framework Programme for Research and Innovation under Grant Agreement No 720270 (Human Brain Project SGA1). AG was a Postdoc fellow of the FWO.

Acknowledgements: We thank A. Coeman, W. Depuydt, M. De Paep, C. Fransen, P. Kayenbergh, G. Meulemans C. Van Eupen and S. Verstraeten for technical assistance. 


\section{References}

Alonso JM, Cudeiro J, Perez R, Gonzalez F, Acuna C. 1993. Influence of layer V of area 18 of the cat visual cortex on responses of cells in layer $V$ of area 17 to stimuli of high velocity. Exp Brain Res 93:363-366.

Anderson JC, Kennedy H, Martin KAC. 2011. Pathways of Attention: Synaptic

Relationships of Frontal Eye Field to V4, Lateral Intraparietal Cortex, and Area 46 in Macaque Monkey. The Journal of Neuroscience 31:10872-10881.

Andrews PR, Johnston GA. 1979. GABA agonists and antagonists. Biochem Pharmacol 28:2697-2702.

Azouvi P, Jacquin-Courtois S, Luaute J. 2017. Rehabilitation of unilateral neglect:

Evidence-based medicine. Ann Phys Rehabil Med 60:191-197.

Balan PF, Gerits A, Mantini D, Vanduffel W. 2017. Selective TMS-induced modulation of functional connectivity correlates with changes in behavior. Neuroimage 149:361-378. Balan PF, Gottlieb J. 2009. Functional significance of nonspatial information in monkey lateral intraparietal area. J Neurosci 29:8166-8176.

Becker E, Karnath HO. 2007. Incidence of visual extinction after left versus right hemisphere stroke. Stroke 38:3172-3174.

Benjamini Y, Krieger AM, Yekutieli D. 2006. Adaptive linear step-up procedures that control the false discovery rate. Biometrika 93:491-507.

Bichot NP, Rossi AF, Desimone R. 2005. Parallel and serial neural mechanisms for visual search in macaque area V4. Science 308:529-534.

Buracas GT, Albright TD. 1999. Covert visual search: a comparison of performance by humans and macaques (Macaca mulatta). Behav Neurosci 113:451-464. 
Burock MA, Buckner RL, Woldorff MG, Rosen BR, Dale AM. 1998. Randomized eventrelated experimental designs allow for extremely rapid presentation rates using functional MRI. Neuroreport 9:3735-3739.

Butefisch CM, Netz J, Wessling M, Seitz RJ, Homberg V. 2003. Remote changes in cortical excitability after stroke. Brain 126:470-481.

Calautti C, Leroy F, Guincestre JY, Marie RM, Baron JC. 2001. Sequential activation brain mapping after subcortical stroke: changes in hemispheric balance and recovery. Neuroreport 12:3883-3886.

Caspari N, Janssens T, Mantini D, Vandenberghe R, Vanduffel W. 2015. Covert shifts of spatial attention in the macaque monkey. J Neurosci 35:7695-7714.

Cazzoli D, Wurtz P, Muri RM, Hess CW, Nyffeler T. 2009. Interhemispheric balance of overt attention: a theta burst stimulation study. Eur J Neurosci 29:1271-1276.

Chee MW, Venkatraman V, Westphal C, Siong SC. 2003. Comparison of block and event-related fMRI designs in evaluating the word-frequency effect. Human Brain Mapping 18:186-193.

Chen Z, Silva AC, Yang J, Shen J. 2005. Elevated endogenous GABA level correlates with decreased fMRI signals in the rat brain during acute inhibition of GABA transaminase. J Neurosci Res 79:383-391.

Chollet F, DiPiero V, Wise RJ, Brooks DJ, Dolan RJ, Frackowiak RS. 1991. The functional anatomy of motor recovery after stroke in humans: a study with positron emission tomography. Ann Neurol 29:63-71. 
Corbetta M. 1998. Frontoparietal cortical networks for directing attention and the eye to visual locations: Identical, independent, or overlapping neural systems? Proceedings of the National Academy of Sciences of the United States of America 95:831-838.

Corbetta M, Akbudak E, Conturo TE, Snyder AZ, Ollinger JM, Drury HA, Linenweber MR, Petersen SE, Raichle ME, Van Essen DC, Shulman GL. 1998. A common network of functional areas for attention and eye movements. Neuron 21:761-773.

Corbetta M, Kincade MJ, Lewis C, Snyder AZ, Sapir A. 2005. Neural basis and recovery of spatial attention deficits in spatial neglect. Nat Neurosci 8:1603-1610.

Corbetta M, Shulman GL. 2011. Spatial neglect and attention networks. Annu Rev Neurosci 34:569-599.

Cramer SC, Nelles G, Benson RR, Kaplan JD, Parker RA, Kwong KK, Kennedy DN, Finklestein SP, Rosen BR. 1997. A functional MRI study of subjects recovered from hemiparetic stroke. Stroke 28:2518-2527.

Cramer SC, Nelles G, Schaechter JD, Kaplan JD, Finklestein SP, Rosen BR. 2001. A functional MRI study of three motor tasks in the evaluation of stroke recovery. Neurorehabil Neural Repair 15:1-8.

DiCiccio TJ, Efron B. 1996. Bootstrap confidence intervals. Statist Sci 11:189-228. Dunn CA, Colby CL. 2010. Representation of the ipsilateral visual field by neurons in the macaque lateral intraparietal cortex depends on the forebrain commissures. $J$ Neurophysiol 104:2624-2633.

Durand JB, Nelissen K, Joly O, Wardak C, Todd JT, Norman JF, Janssen P, Vanduffel W, Orban GA. 2007. Anterior regions of monkey parietal cortex process visual 3D shape. Neuron 55:493-505. 
Efron B. 1987. Better Bootstrap Confidence Intervals. Journal of the American Statistical Association 82:171-185.

Efron B, Tibshirani R. 1996. An introduction to the bootstrap. New York u.a.: Chapman \& Hall.

Ekstrom LB, Roelfsema PR, Arsenault JT, Bonmassar G, Vanduffel W. 2008. Bottom-up dependent gating of frontal signals in early visual cortex. Science 321:414-417.

Falkner AL, Krishna BS, Goldberg ME. 2010. Surround suppression sharpens the priority map in the lateral intraparietal area. J Neurosci 30:12787-12797.

Felleman DJ, Van Essen DC. 1991. Distributed hierarchical processing in the primate cerebral cortex. Cereb Cortex 1:1-47.

Fergus A, Lee KS. 1997. GABAergic regulation of cerebral microvascular tone in the rat. J Cereb Blood Flow Metab 17:992-1003.

Ferry AT, Ongur D, An X, Price JL. 2000. Prefrontal cortical projections to the striatum in macaque monkeys: evidence for an organization related to prefrontal networks. $\mathrm{J}$ Comp Neurol 425:447-470.

Fischl B. 2012. FreeSurfer. Neurolmage 62:774-781.

Hupe JM, James AC, Payne BR, Lomber SG, Girard P, Bullier J. 1998. Cortical feedback improves discrimination between figure and background by V1, V2 and V3 neurons. Nature 394:784-787.

Ibos G, Duhamel JR, Ben Hamed S. 2013. A functional hierarchy within the parietofrontal network in stimulus selection and attention control. J Neurosci 33:83598369. 
Jacobs KM, Donoghue JP. 1991. Reshaping the cortical motor map by unmasking latent intracortical connections. Science 251:944-947.

Janssens T, Zhu Q, Popivanov ID, Vanduffel W. 2014. Probabilistic and single-subject retinotopic maps reveal the topographic organization of face patches in the macaque cortex. Journal of Neuroscience 34:10156-10167.

Johansen-Berg H. 2007. Functional imaging of stroke recovery: what have we learnt and where do we go from here? Int J Stroke 2:7-16.

Kagan I, Iyer A, Lindner A, Andersen RA. 2010. Space representation for eye movements is more contralateral in monkeys than in humans. Proc Natl Acad Sci U S A 107:7933-7938.

Karnath HO, Rorden C. 2012. The anatomy of spatial neglect. Neuropsychologia 50:1010-1017.

Kinsbourne M. 1977. Hemi-neglect and hemisphere rivalry. Advances in Neurology 18:41-49.

Kolster H, Janssens T, Orban GA, Vanduffel W. 2014. The retinotopic organization of macaque occipitotemporal cortex anterior to $\mathrm{V} 4$ and caudoventral to the middle temporal (MT) cluster. Journal of Neuroscience 34:10168-10191.

Kolster H, Mandeville JB, Arsenault JT, Ekstrom LB, Wald LL, Vanduffel W. 2009. Visual field map clusters in macaque extrastriate visual cortex. J Neurosci 29:70317039.

Leonards U, Rettenbach R, Sireteanu R. 1998. Parallel visual search is not always effortless. Brain Res Cogn Brain Res 7:207-213. 
Lomber SG, Malhotra S, Sprague JM. 2007. Restoration of acoustic orienting into a cortically deaf hemifield by reversible deactivation of the contralesional superior colliculus: the acoustic "Sprague Effect". J Neurophysiol 97:979-993.

Mandeville JB, Marota JJ. 1999. Vascular filters of functional MRI: spatial localization using BOLD and CBV contrast. Magn Reson Med 42:591-598.

Marshall RS, Perera GM, Lazar RM, Krakauer JW, Constantine RC, DeLaPaz RL. 2000. Evolution of cortical activation during recovery from corticospinal tract infarction. Stroke 31:656-661.

Moore T, Armstrong KM. 2003. Selective gating of visual signals by microstimulation of frontal cortex. Nature 421:370-373.

Nakayama K, Silverman GH. 1986. Serial and parallel processing of visual feature conjunctions. Nature 320:264-265.

Nelissen K, Borra E, Gerbella M, Rozzi S, Luppino G, Vanduffel W, Rizzolatti G, Orban GA. 2011. Action observation circuits in the macaque monkey cortex. J Neurosci 31:3743-3756.

Nelissen K, Luppino G, Vanduffel W, Rizzolatti G, Orban GA. 2005. Observing others: multiple action representation in the frontal lobe. Science 310:332-336.

Ogawa T, Komatsu H. 2004. Target selection in area V4 during a multidimensional visual search task. J Neurosci 24:6371-6382.

Payne BR, Lomber SG. 1999. A method to assess the functional impact of cerebral connections on target populations of neurons. J Neurosci Methods 86:195-208. Ponce CR, Lomber SG, Born RT. 2008. Integrating motion and depth via parallel pathways. Nat Neurosci 11:216-223. 
Premereur E, Vanduffel W, Roelfsema PR, Janssen P. 2012. Frontal eye field microstimulation induces task-dependent gamma oscillations in the lateral intraparietal area. J Neurophysiol 108:1392-1402.

Pruessmann KP, Weiger M, Scheidegger MB, Boesiger P. 1999. SENSE: sensitivity encoding for fast MRI. Magn Reson Med 42:952-962.

Reveley C, Gruslys A, Ye FQ, Glen D, Samaha J, B ER, Saad Z, A KS, Leopold DA, Saleem KS. 2016. Three-Dimensional Digital Template Atlas of the Macaque Brain. Cereb Cortex.

Rizzolatti G, Luppino G. 2001. The cortical motor system. Neuron 31:889-901.

Rushmore RJ, Payne BR, Lomber SG. 2005. Functional impact of primary visual cortex deactivation on subcortical target structures in the thalamus and midbrain. J Comp Neurol 488:414-426.

Saj A, Cojan Y, Vocat R, Luaute J, Vuilleumier P. 2013. Prism adaptation enhances activity of intact fronto-parietal areas in both hemispheres in neglect patients. Cortex 49:107-119.

Salin PA, Bullier J. 1995. Corticocortical connections in the visual system: structure and function. Physiol Rev 75:107-154.

Schall J, Morel A, King D, Bullier J. 1995. Topography of visual cortex connections with frontal eye field in macaque: convergence and segregation of processing streams. The Journal of Neuroscience 15:4464-4487.

Seitz RJ, Hoflich P, Binkofski F, Tellmann L, Herzog H, Freund HJ. 1998. Role of the premotor cortex in recovery from middle cerebral artery infarction. Arch Neurol 55:10811088. 
Shimizu T, Hosaki A, Hino T, Sato M, Komori T, Hirai S, Rossini PM. 2002. Motor cortical disinhibition in the unaffected hemisphere after unilateral cortical stroke. Brain 125:1896-1907.

Simpson GV, Weber DL, Dale CL, Pantazis D, Bressler SL, Leahy RM, Luks TL. 2011. Dynamic activation of frontal, parietal, and sensory regions underlying anticipatory visual spatial attention. J Neurosci 31:13880-13889.

Sireteanu R, Rettenbach R. 1995. Perceptual learning in visual search: fast, enduring, but non-specific. Vision Res 35:2037-2043.

Sireteanu R, Rettenbach R. 2000. Perceptual learning in visual search generalizes over tasks, locations, and eyes. Vision Res 40:2925-2949.

Stanton GB, Bruce CJ, Goldberg ME. 1995. Topography of projections to posterior cortical areas from the macaque frontal eye fields. J Comp Neurol 353:291-305.

Thimm M, Fink GR, Kust J, Karbe H, Willmes K, Sturm W. 2009. Recovery from hemineglect: differential neurobiological effects of optokinetic stimulation and alertness training. Cortex 45:850-862.

Tootell RB, Nelissen K, Vanduffel W, Orban GA. 2004. Search for color 'center(s)' in macaque visual cortex. Cereb Cortex 14:353-363.

Umarova RM, Nitschke K, Kaller CP, Kloppel S, Beume L, Mader I, Martin M, Hennig J, Weiller C. 2016. Predictors and signatures of recovery from neglect in acute stroke. Ann Neurol 79:673-686.

Umarova RM, Saur D, Kaller CP, Vry MS, Glauche V, Mader I, Hennig J, Weiller C. 2011. Acute visual neglect and extinction: distinct functional state of the visuospatial attention system. Brain 134:3310-3325. 
Van Essen DC, Lewis JW, Drury HA, Hadjikhani N, Tootell RB, Bakircioglu M, Miller MI. 2001. Mapping visual cortex in monkeys and humans using surface-based atlases. Vision Res 41:1359-1378.

Vanduffel W, Fize D, Mandeville JB, Nelissen K, Van Hecke P, Rosen BR, Tootell RB, Orban GA. 2001. Visual motion processing investigated using contrast agent-enhanced fMRI in awake behaving monkeys. Neuron 32:565-577.

Vanduffel W, Payne BR, Lomber SG, Orban GA. 1997. Functional impact of cerebral connections. Proc Natl Acad Sci U S A 94:7617-7620.

Vossel S, Eschenbeck P, Weiss PH, Weidner R, Saliger J, Karbe H, Fink GR. 2011. Visual extinction in relation to visuospatial neglect after right-hemispheric stroke: quantitative assessment and statistical lesion-symptom mapping. J Neurol Neurosurg Psychiatry 82:862-868.

Ward NS, Brown MM, Thompson AJ, Frackowiak RS. 2003. Neural correlates of outcome after stroke: a cross-sectional fMRI study. Brain 126:1430-1448.

Wardak C, Ben Hamed S, Olivier E, Duhamel J-R. 2012. Differential effects of parietal and frontal inactivations on reaction times distributions in a visual search task. Frontiers in Integrative Neuroscience 6.

Wardak C, Olivier E, Duhamel JR. 2002. Saccadic target selection deficits after lateral intraparietal area inactivation in monkeys. J Neurosci 22:9877-9884.

Wardak C, Olivier E, Duhamel JR. 2004. A deficit in covert attention after parietal cortex inactivation in the monkey. Neuron 42:501-508.

Wardak C, Olivier E, Duhamel JR. 2011. The relationship between spatial attention and saccades in the frontoparietal network of the monkey. Eur J Neurosci 33:1973-1981. 
Wardak C, Vanduffel W, Orban GA. 2010. Searching for a salient target involves frontal regions. Cereb Cortex 20:2464-2477.

Weiller C, Chollet F, Friston KJ, Wise RJ, Frackowiak RS. 1992. Functional

reorganization of the brain in recovery from striatocapsular infarction in man. Ann Neurol 31:463-472.

Wilke M, Kagan I, Andersen RA. 2012. Functional imaging reveals rapid reorganization of cortical activity after parietal inactivation in monkeys. Proc Natl Acad Sci U S A 109:8274-8279.

Wilke M, Kagan I, Andersen RA. 2012. Functional imaging reveals rapid reorganization of cortical activity after parietal inactivation in monkeys. Proc Natl Acad Sci U S A 109:8274-8279.

Worsley KJ, Friston KJ. 1995. Analysis of fMRI time-series revisited--again. Neuroimage 2:173-181.

Zhang J, Mueller ST. 2005. A note on ROC analysis and non-parametric estimate of sensitivity. Psychometrika 70:203-212.

\section{Tables}

Table 1 Number of runs used in the analysis per task per monkey and per condition

\begin{tabular}{|l|c|c|}
\hline \multicolumn{1}{|c|}{ MONKEY } & M9 & M10 \\
\hline TASK & CJ \& DET & CJ \& DET \\
\hline
\end{tabular}




\begin{tabular}{|l|c|c|}
\hline Pre-inactivation & 32 & 37 \\
\hline SALINE & 36 & 36 \\
\hline MUSCIMOL & 56 & 52 \\
\hline
\end{tabular}

Table 2 Kruskal-Wallis statistics for d-prime and a-prime

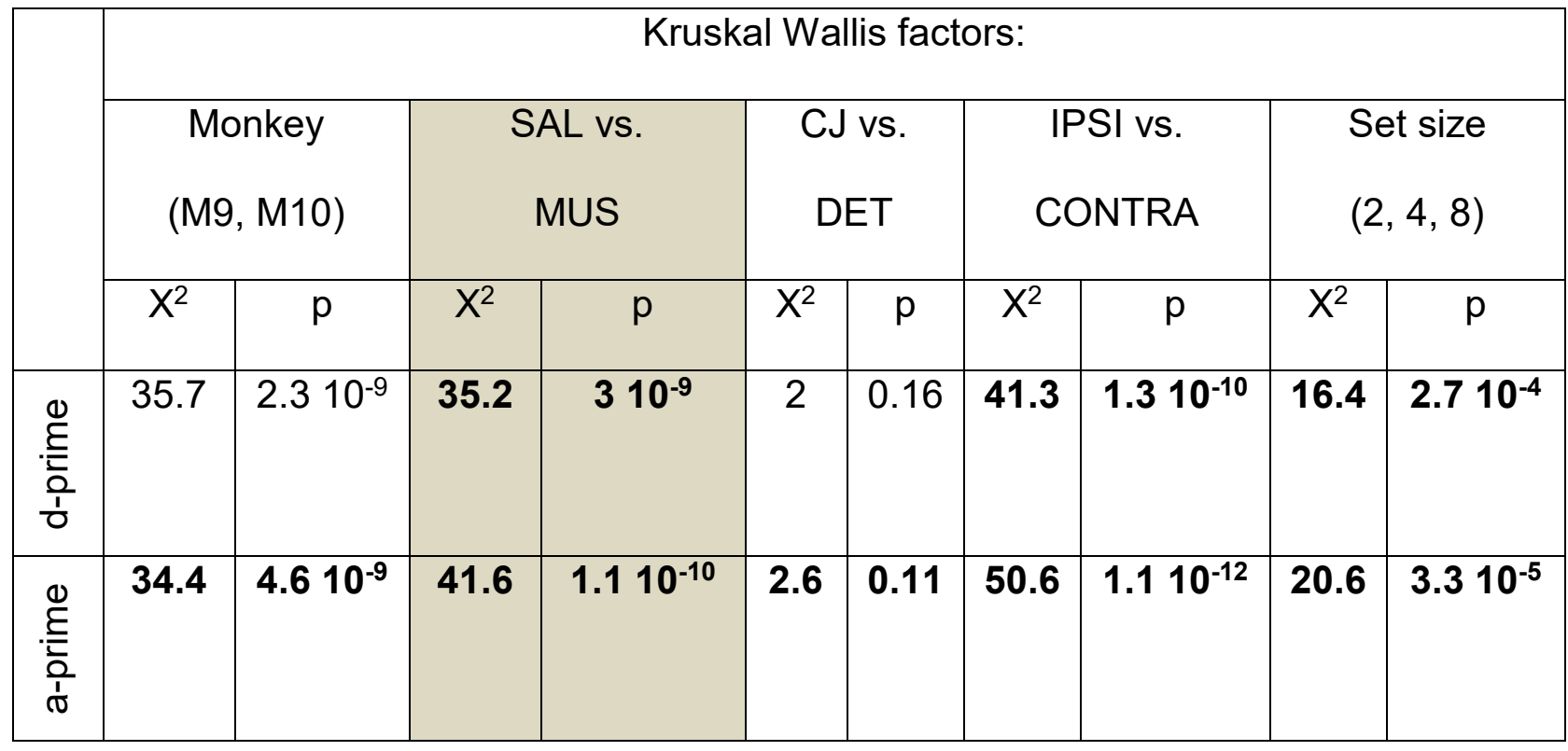

Degrees of freedom: $(1,334)$ in $M 9,(1,330)$ in $M 10$ and $(1,664)$ in $M 9 \& M 10$, for all factors, except set size: $(2,334)$ in $M 9,(2,330)$ in $M 10$ and $(2,664)$ in $M 9$ \& M10. $X^{2}=$ chi-square values. The set-size effect is significant only for set size 2 vs. set size 8 . SAL - saline; MUS - muscimol; IPSI - ipsilesional; CONTRA - contralesional. Bold characters underline significant effects.

Table 3 Supplementary Wilcoxon rank sum test Saline vs. Muscimol for d-prime

\begin{tabular}{|c|c|}
\hline $\mathscr{N}$ & Wilcoxon rank sum test Saline vs. Muscimol: \\
$\frac{\mathscr{N}}{3}$ & d-prime \\
$\frac{\mathrm{O}}{\mathcal{D}}$ & \\
\hline
\end{tabular}




\begin{tabular}{|c|c|c|c|c|}
\hline \multirow{2}{*}{} & \multicolumn{4}{|c|}{ a-prime } \\
\cline { 2 - 5 } & \multicolumn{2}{|c|}{ CJ } & $\mathrm{P}$ & $\mathrm{P}$ \\
\cline { 2 - 5 } & $\mathrm{P}$ & $\mathrm{P}$ & $\mathrm{P}$ & \\
& ipsilesional & contralesional & ipsilesional & contralesional \\
\hline M9 & $\mathbf{7 . 3 1 0 ^ { - 3 }}$ & $\mathbf{2 . 6 1 0 ^ { - 3 }}$ & $\mathbf{1 . 4 1 0 ^ { - 3 }}$ & $1.310^{-2}$ \\
& $\mathbf{4 . 3 1 0 ^ { - 3 }}$ & $\mathbf{1 . 2 1 0 ^ { - 3 }}$ & $\mathbf{1 . 8 1 0 ^ { - 3 }}$ & $\mathbf{7 . 9 1 0 ^ { - 3 }}$ \\
\hline M10 & $\mathbf{5 . 6 1 0 ^ { - 3 }}$ & $\mathbf{6 . 6 1 0 ^ { - 3 }}$ & 0.52 & $3.210^{-2}$ \\
& $\mathbf{5 1 0 ^ { - 4 }}$ & $\mathbf{6 . 1 1 0 ^ { - 3 }}$ & 0.34 & $2.510^{-2}$ \\
\hline
\end{tabular}

\section{Figure Captions}

Figure 1. Search and detection task: stimuli and temporal organization of trials and time series.

A. Examples of stimuli for an 8-item configuration of the search and the detection task. A dark red fixation point $(\mathrm{CJ})$ cued the monkey to respond to the 'target' indicated by a dotted oval. When the fixation point was green, the monkey had to detect the brightening event (DET). The dashed and dotted shapes are displayed only for clarity. 2 and 4-item configurations were also tested but not shown.

B. Examples of trial types and temporal organization of CJ trials. The fixation point was continuously present and after 1000-1250 ms, the stimuli appeared for $1300 \mathrm{~ms}$ (grey part of timeline). A target was presented in $50 \%$ of the trials. To be rewarded the monkey had to manually respond between $100 \mathrm{~ms}$ after stimulus onset and before 
stimulus end. To equalize visual presentation, an irrelevant brightening event occurred for 300 ms starting $1000 \mathrm{~ms}$ after stimulus onset in $50 \%$ of the trials.

C. Examples of trial types and temporal organization of DET. A green fixation point was always present, and after 1000-1250 ms the stimuli were shown for $1300 \mathrm{~ms}$. After $50-$ $400 \mathrm{~ms}$, a brightening event occurred for $300 \mathrm{~ms}$ in $50 \%$ of the trials. The monkey had to manually respond within $100 \mathrm{~ms}$ after brightening onset and before stimulus end to be rewarded. In $50 \%$ of the brightening trials and in $50 \%$ of the trials without brightening, the target of the search task appeared but had to be ignored. Yellow squares mark the reward period ( $B$ and $C)$

D. Example of temporal organization of time series. A time series started with 10 TRs fixation (20 s) followed by 15 TRs (30 s) of search or detection. When search came first, 15 TRs of detection followed and vice versa. This sequence was repeated four times (160 TRs; 240 s).

Figure 2. Schematic overview of injection sites in LIP and behavioral effects of LIP inactivation.

A. View on a representative para-horizontal section (T1-weighted image) orthogonal to the grid at $9 \mathrm{~mm}$ below the dura. The black crosses indicate the center of the recording chamber (yellow circles). The small yellow dots represent the grid holes and the black dots the injection sites. Left: View of the injection sites in the lateral bank of the left IPS of M9. Location of some sites may appear to be in the medial wall or white matter but as the curvature of the IPS changes as function of depth, injections through these grid holes were actually administered in grey matter (i.e. the injections may have been made 
out of the plane shown in the section). Right: View of the injection sites in the lateral bank of the right IPS of M10. Same conventions as above (CS, central sulcus; IPS, intraparietal sulcus; STS, superior temporal sulcus; A, anterior; P, posterior; M, medial; $\mathrm{L}$, lateral; $\mathrm{LH}$, left hemisphere; $\mathrm{RH}$, right hemisphere). The red patches overlapping the injection sites mark the projection of the fMRI activations (contrast $C J$ vs. fixation, preinjection experiments; T $>3.1$, FDR corrected $p=0.05$ ) of all planes parallel to the presented plane. Areas that appear on the opposite side of the IPS (due to the nonorthogonal trajectory of the IPS relative to the projection plane) are shaded in lighter red.

B. D-prime and reaction times (C) with the target in the ipsi- (circles) and contralesional (triangles) hemifield for M9 (left panel) and M10 (right panel), saline and muscimol data,

CJ task. Error bars represent SEM across runs $(n=56$ and 52 for the muscimol condition in M9 and M10 respectively; $\mathrm{n}=36$ and 36 for the saline condition in M9 and M10, respectively). Stars indicate significant differences between pairs of conditions linked with the black lines (Tables 3 and $4 \mathrm{~S}$ ).

Figure 3. Muscimol-induced changes in local fMRI activity after LIP injections. Coronal slices (T1-weighted images) showing activity (t-score maps) of MVSD vs. fixation $(P<0.05$, FDR corrected, $T>3.1)$ after injecting saline $(A ;$ blue $)$ and muscimol (B; red) in M9 (left) and M10 (right). Insert: percent fMRI signal change relative to fixation at the local maximum in LIP ( ${ }^{*} \mathrm{P}<110^{-4}$; error bars represent SEM across runs ( $\mathrm{n}=56$ and 52 for the muscimol condition in M9 and M10 respectively; $\mathrm{n}=32$ and 36 for the saline condition in M9 and M10, respectively). Coronal slices show patches within 
LIP with decreased fMRI activity in the injected hemisphere (blue) and increased fMRI activity in the contralateral hemisphere (red) for M9 (C) and M10 (E) $(T \geq 3.1, P<0.05$, FDR corrected). Yellow marks the LIP ROI as defined in the paragraph "Region of interest analysis". Changes in PSC (muscimol (Mus) vs. saline (Sal)) for M9 (D) and M10 (F) (mean and $\mathrm{Cl}: \mathrm{p}=3 \mathrm{10}^{-5}$ ) correspond to those patches shown in $\mathrm{C}$ and $\mathrm{E}$ (using the same color-code).

Figure 4. Muscimol-induced changes in cortical MVSD-related fMRI activity. Flattened representation of ipsilesional $(A)$ and contralesional $(B)$ hemispheres of both monkeys overlain with the network activated during the MVSD task for muscimol (redyellow t-score maps; $\mathrm{P}<0.05$, FDR corrected, $\mathrm{T}>3.1$ ) and saline injections (blue outlines; $P<0.05$, FDR corrected, $T>3.1$ ). White lines are outlines of regions involved in the attention task network. The scale bar equals $4 \mathrm{~mm}$.

Figure 5. Comparison between muscimol and saline for the MVSD task-related fMRI activity. Flattened representation of ipsilesional (A) and contralesional (B) hemispheres of both monkeys overlain with the search task network activated more strongly after muscimol compared to saline (red-yellow t-score maps; $\mathrm{P}<0.05$, FDR corrected, $\mathrm{T}>3.1$ ). White lines are outlines of regions involved in the attention task network. The scale bar equals $4 \mathrm{~mm}$.

Figure 6. Differences in percent fMRI signal change between muscimol and saline for ipsi- and contralesional cortical regions for the MVSD task. Differences in 
percent signal change (PSC) between muscimol and saline is plotted for each ROI for M9 (A) and M10 (B). When the difference in PSC is greater than zero, the PSC in that ROI is increased after muscimol injections in LIP (red). When the difference in PSC is smaller than zero, the PSC in that ROI is higher after saline injections in LIP (blue). Data points where differences in PSC do not differ from zero are black. Each data point represents mean and $\mathrm{Cl}\left(\mathrm{p}=210^{-6}\right)$. Areas that show a consistent result between monkeys are indicated with yellow shading on their labels. LIP represents pooled LIPa and LIPi.

Figure 7. Correlations between behavioral performance and task-based fMRI activity. Flattened representation of ipsilesional (A) and contralesional (B) hemispheres of both monkeys overlain with the differences in z-scores (r-to-z transformed; muscimol - saline) of the correlation between percent correct performance levels during the MVSD task and the corresponding beta values generated by the GLM analyses. Regions with a stronger correlation between percent correct and beta values after muscimol are shown in red-yellow $(\mathrm{P}<0.05 \mathrm{FDR}$ corrected, $\mathrm{T}>3.1)$. White lines are ROI outlines. The scale bar equals $4 \mathrm{~mm}$.

Figure 8. Regions with increased behavioral correlation and increased fMRI activation after muscimol injections. Increases in the fMRI activations for regions also showing increased correlation with behavior after LIP inactivation are plotted for the ipsi- (A) and contralesional (C) hemispheres of M9 (red circle) and M10 (blue triangle). Symbols are filled where FDR corrected-level (all above dashed horizontal black line) 
was reached. Regions showing consistent results across subjects are shown by lines connecting the symbols: continuous mangenta lines where both subjects show significant results; dashed lines where only one (light blue) or none of the monkeys (green) show significant $(t>3.1)$ results. Areal labels are shaded using the same colorcode as the lines. Increased correlation with behavior where fMRI activations also increased after muscimol injections are plotted for the ipsi- (B) and contralesional (D) hemispheres of M9 and M10. Same conventions as in A and C. Regions not showing significant differences at uncorrected level are not listed. 


\section{Figure 1}

A Stimuli and tasks
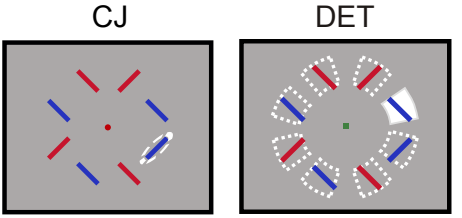

$\mathrm{B} C \mathrm{CJ}$
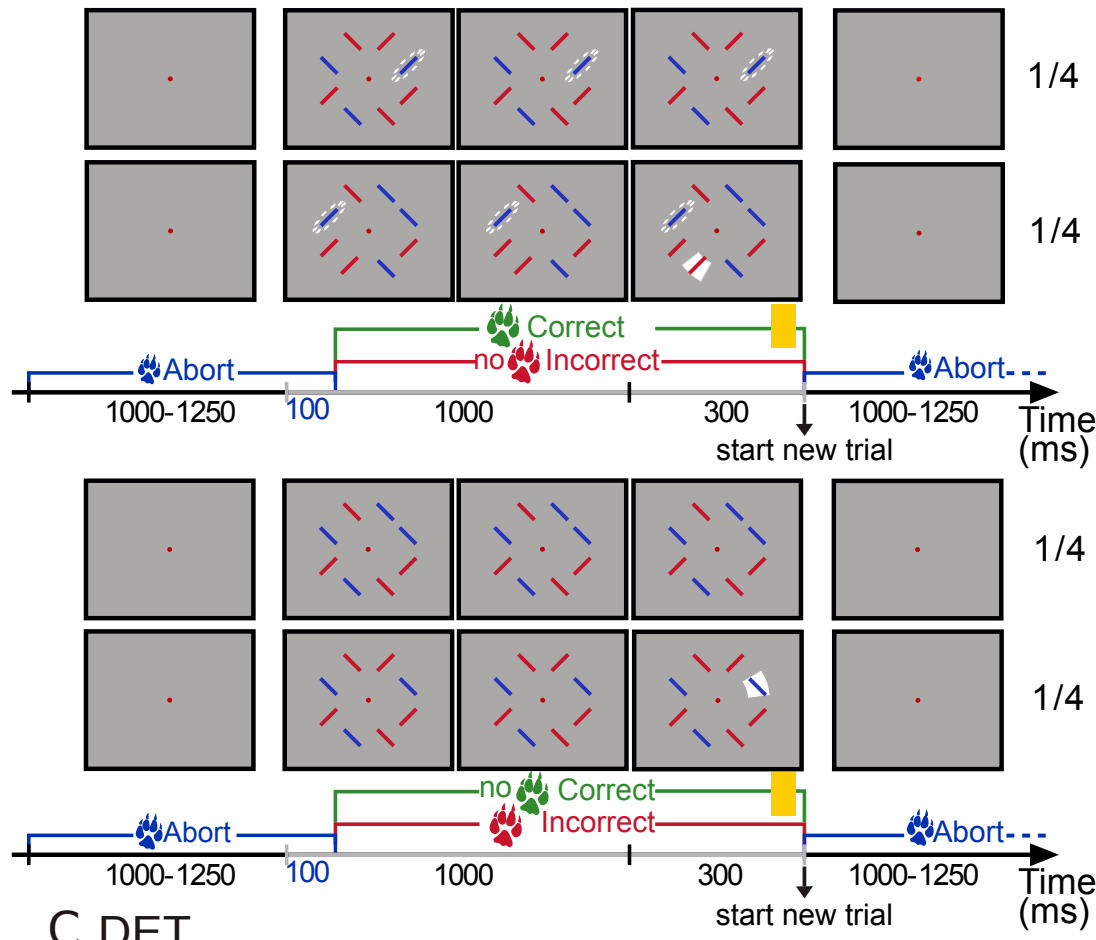

C DET
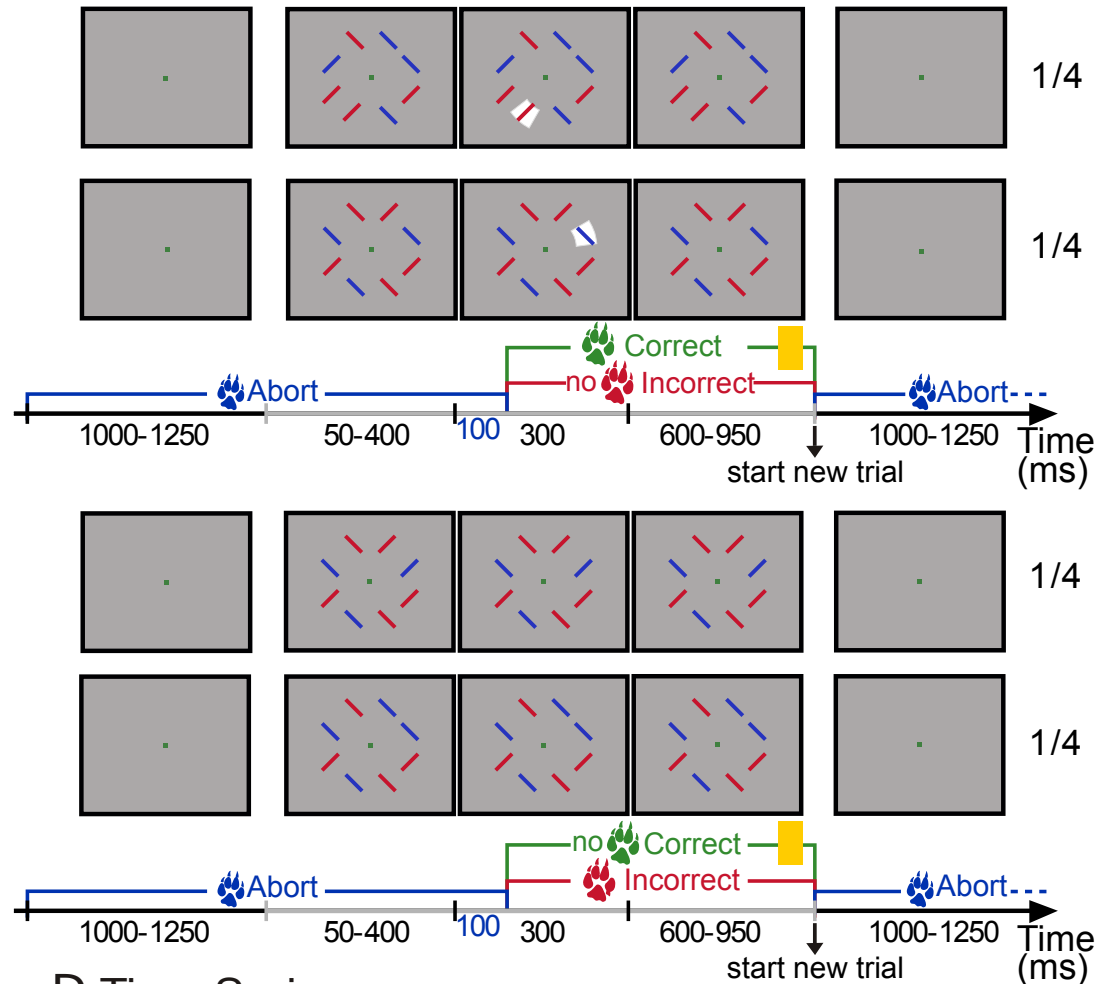

D Time Series start new trial (ms)
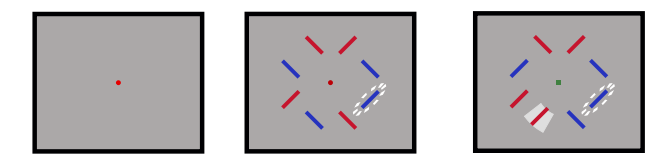

$4 x \frac{\text { fixation }}{10 \text { TRs }}$

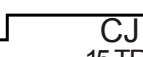

CJ DET $\left\ulcorner^{---}\right.$ 
A

Figure 2

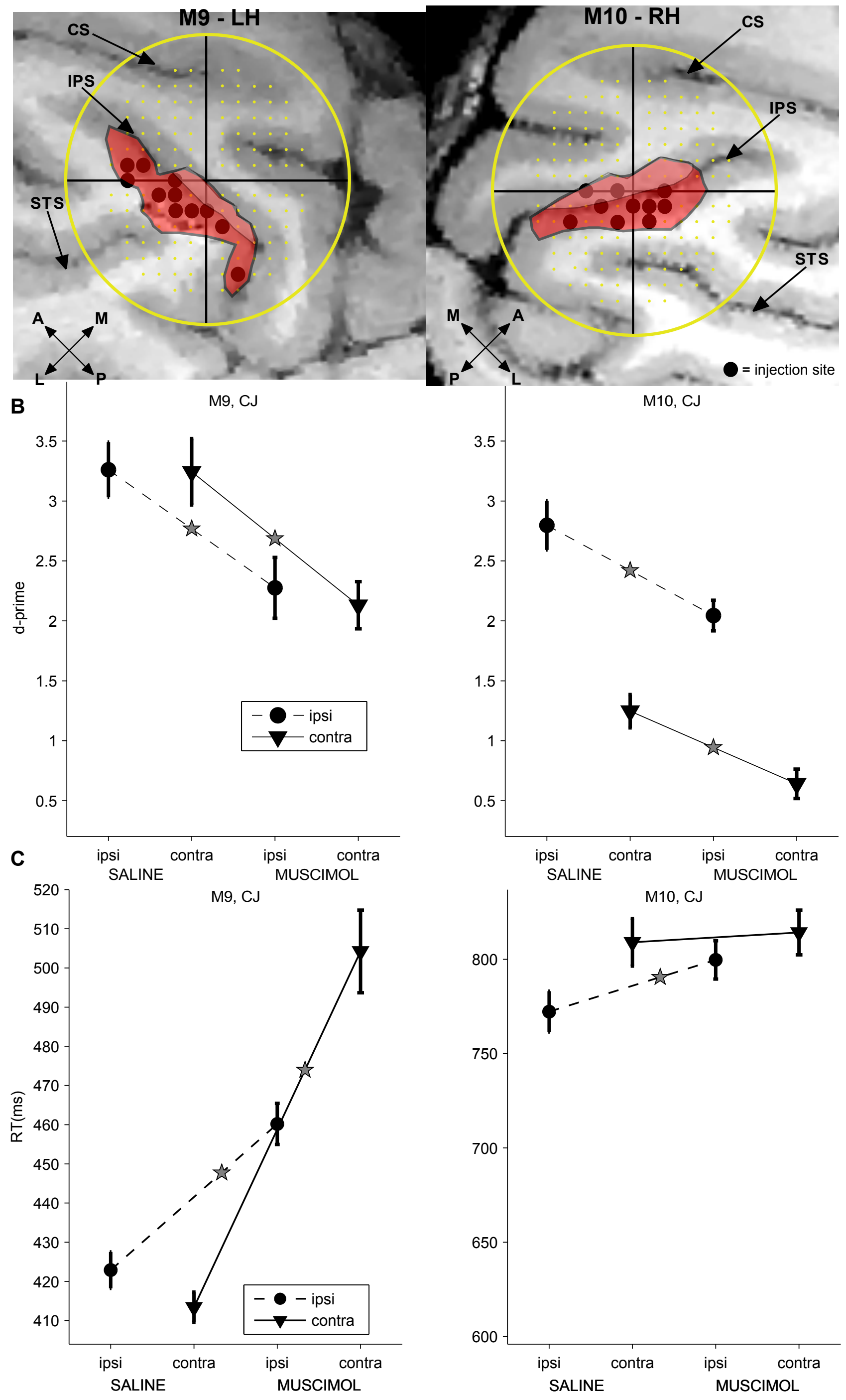


Figure 2S1

A

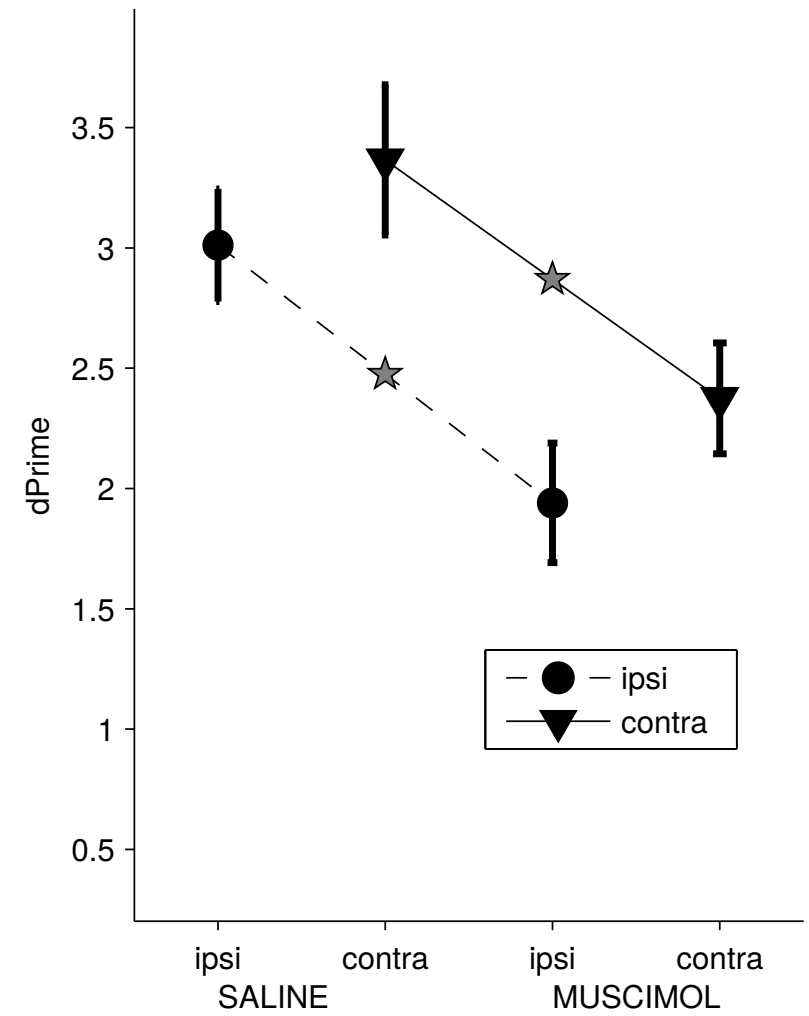

B

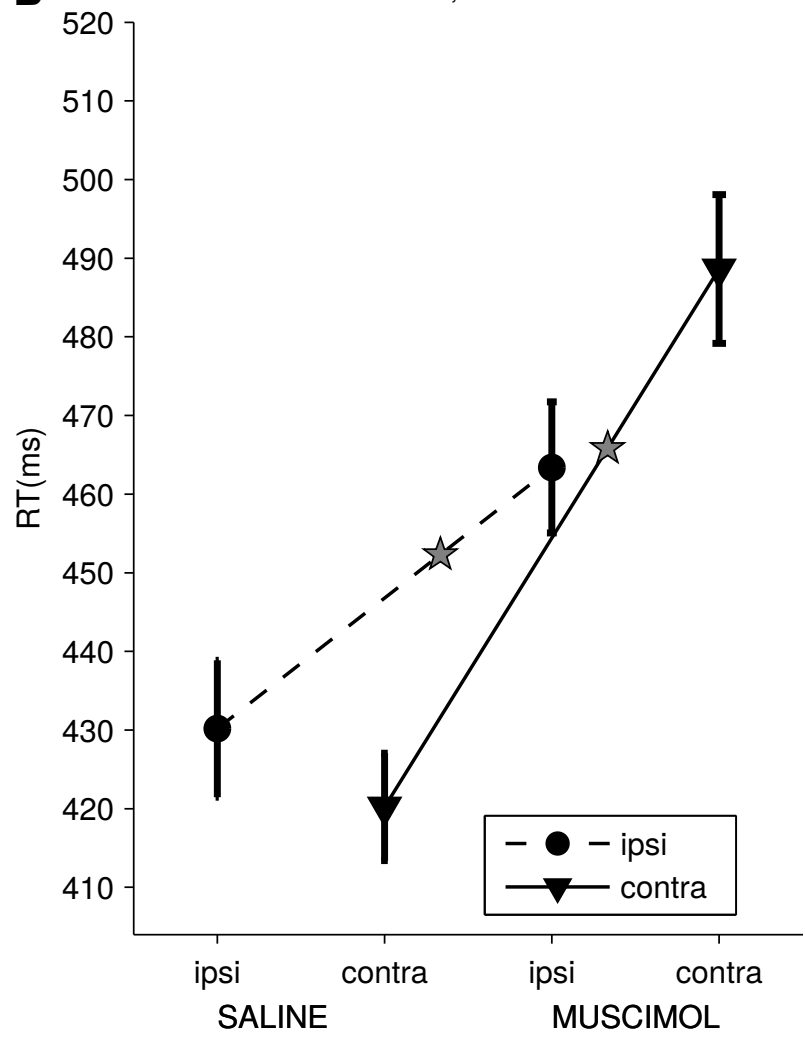

M10, DET

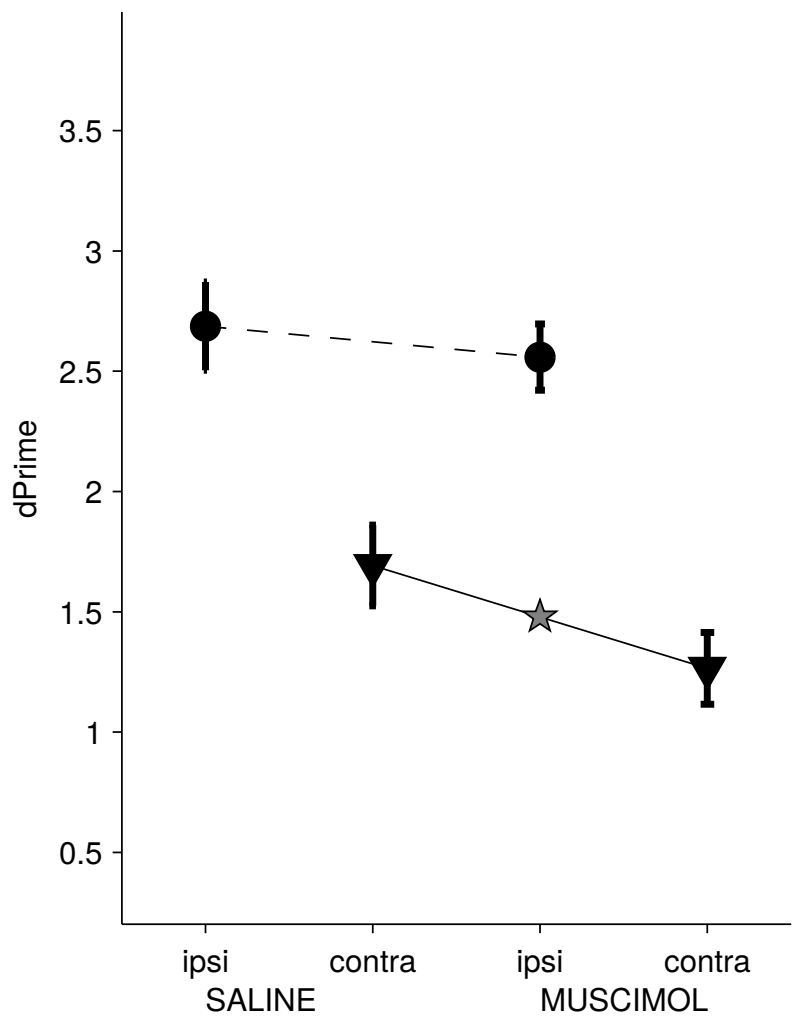

M10, DET

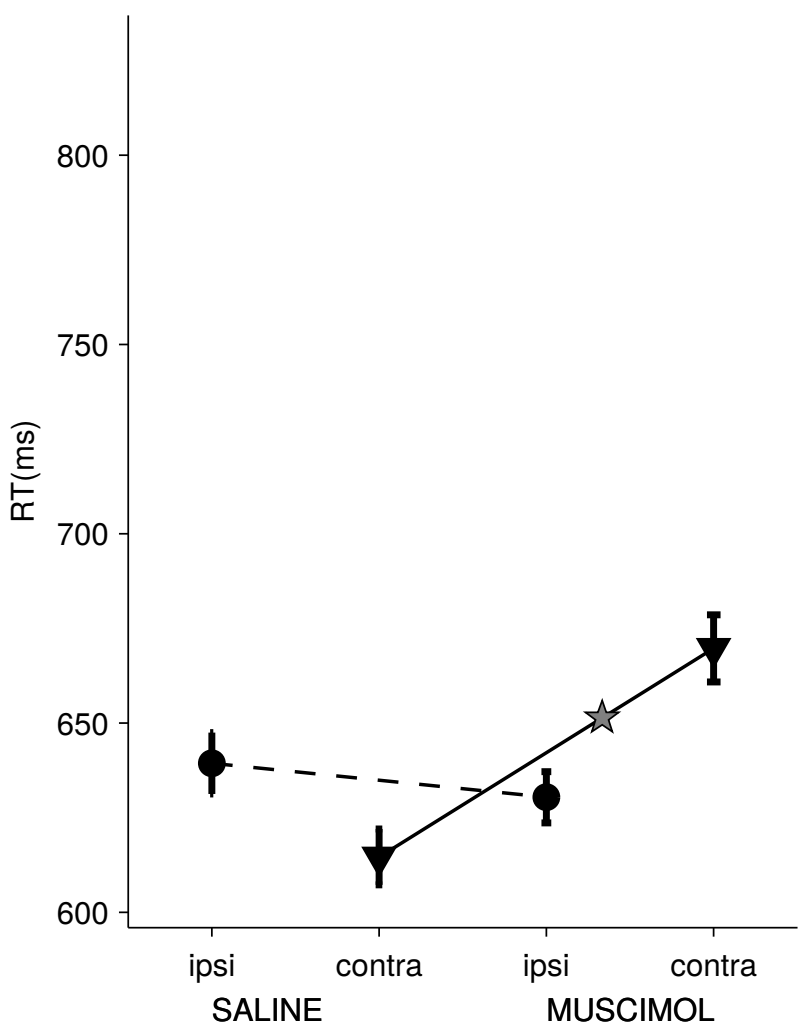




\section{Figure 2S2}
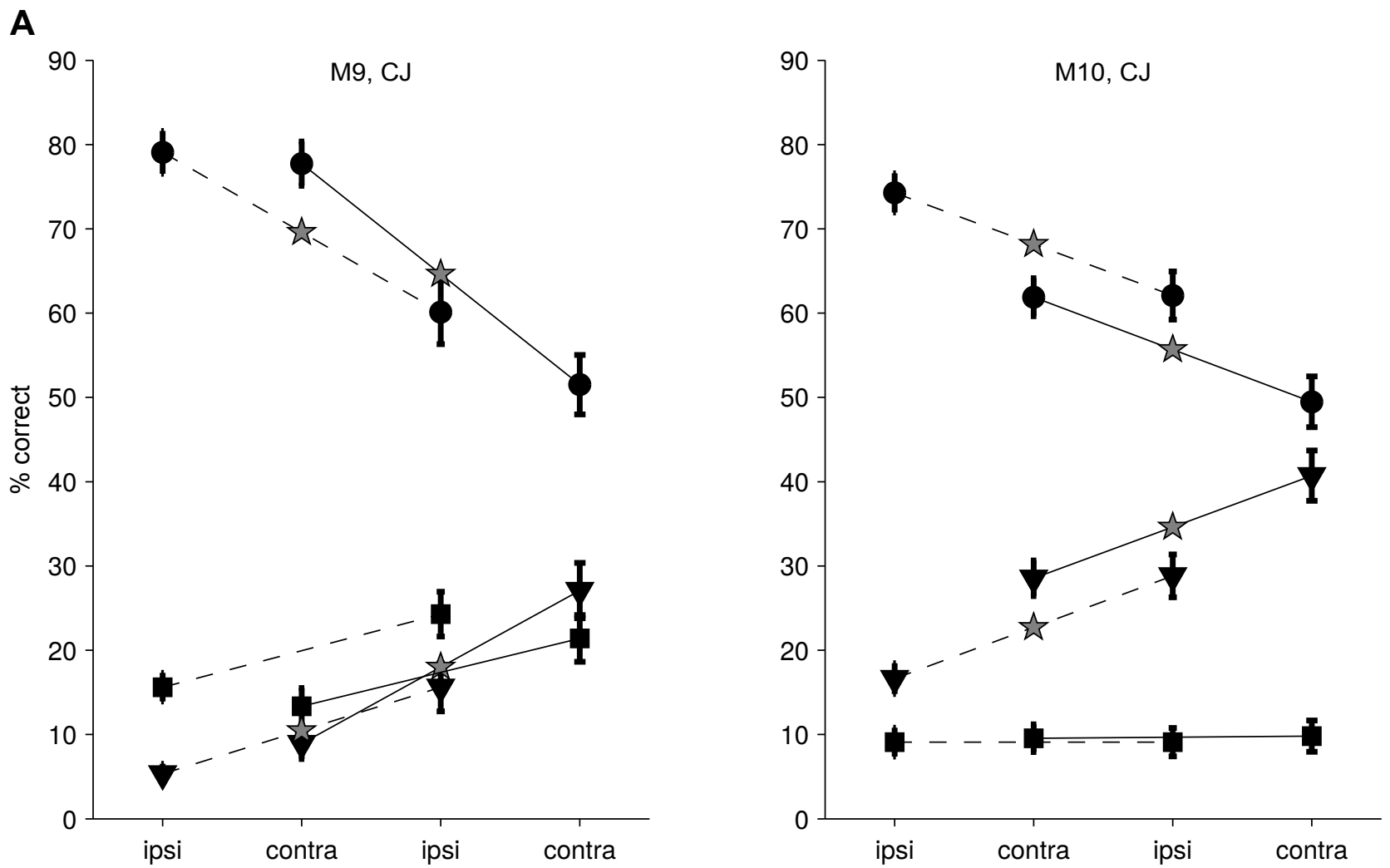

B
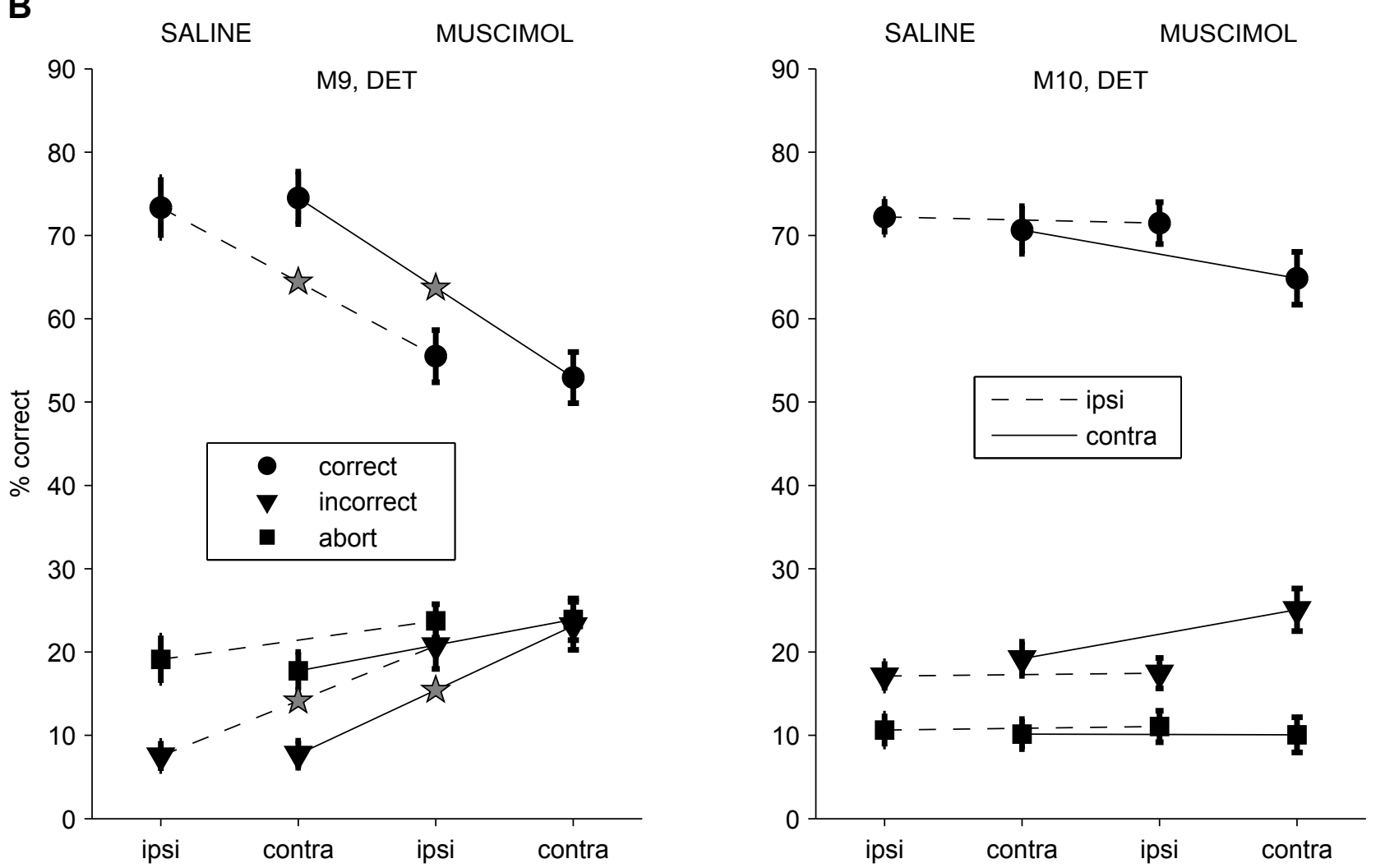

SALINE

MUSCIMOL

MUSCIMOL 
Figure 3

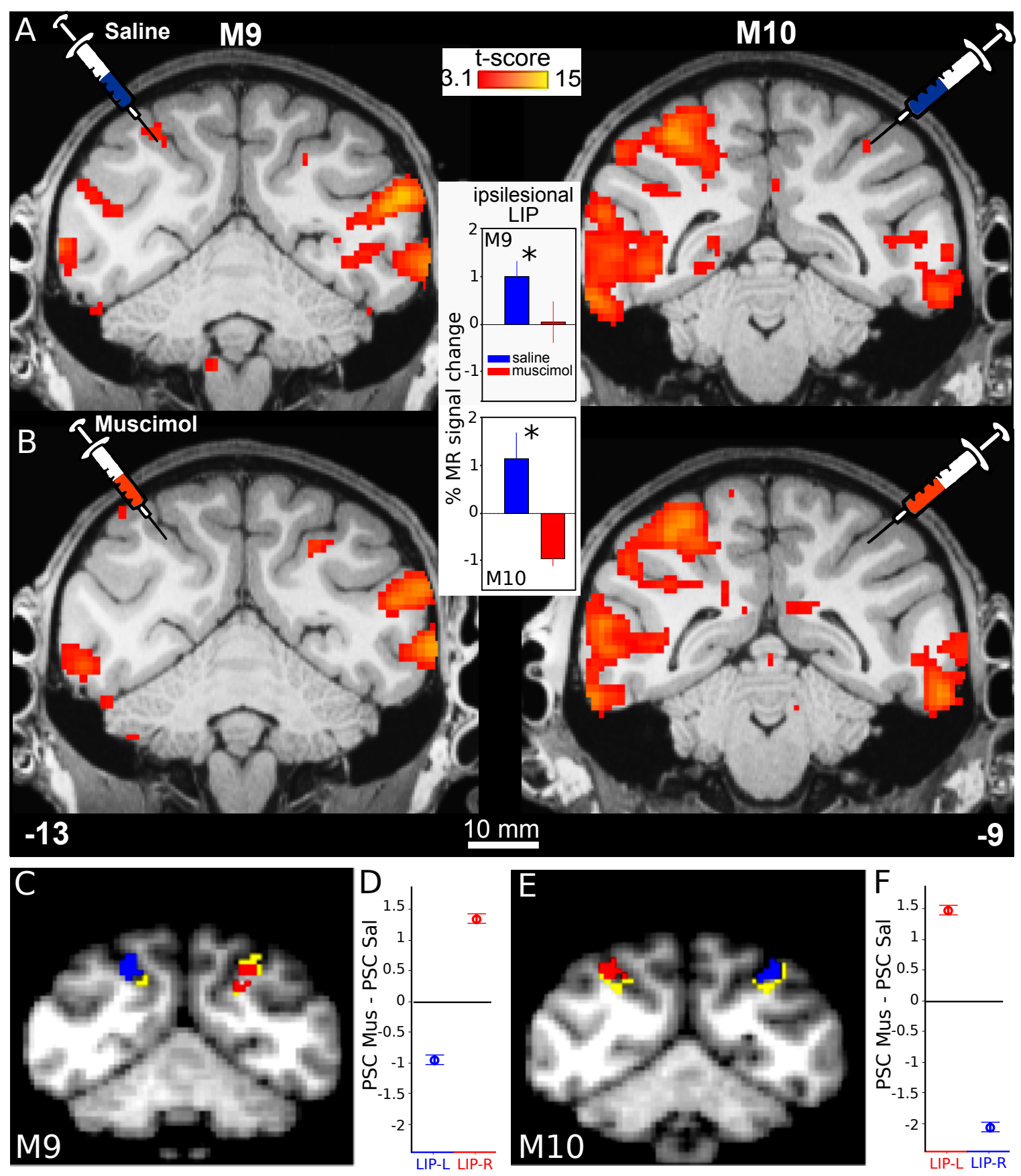


Fig 4

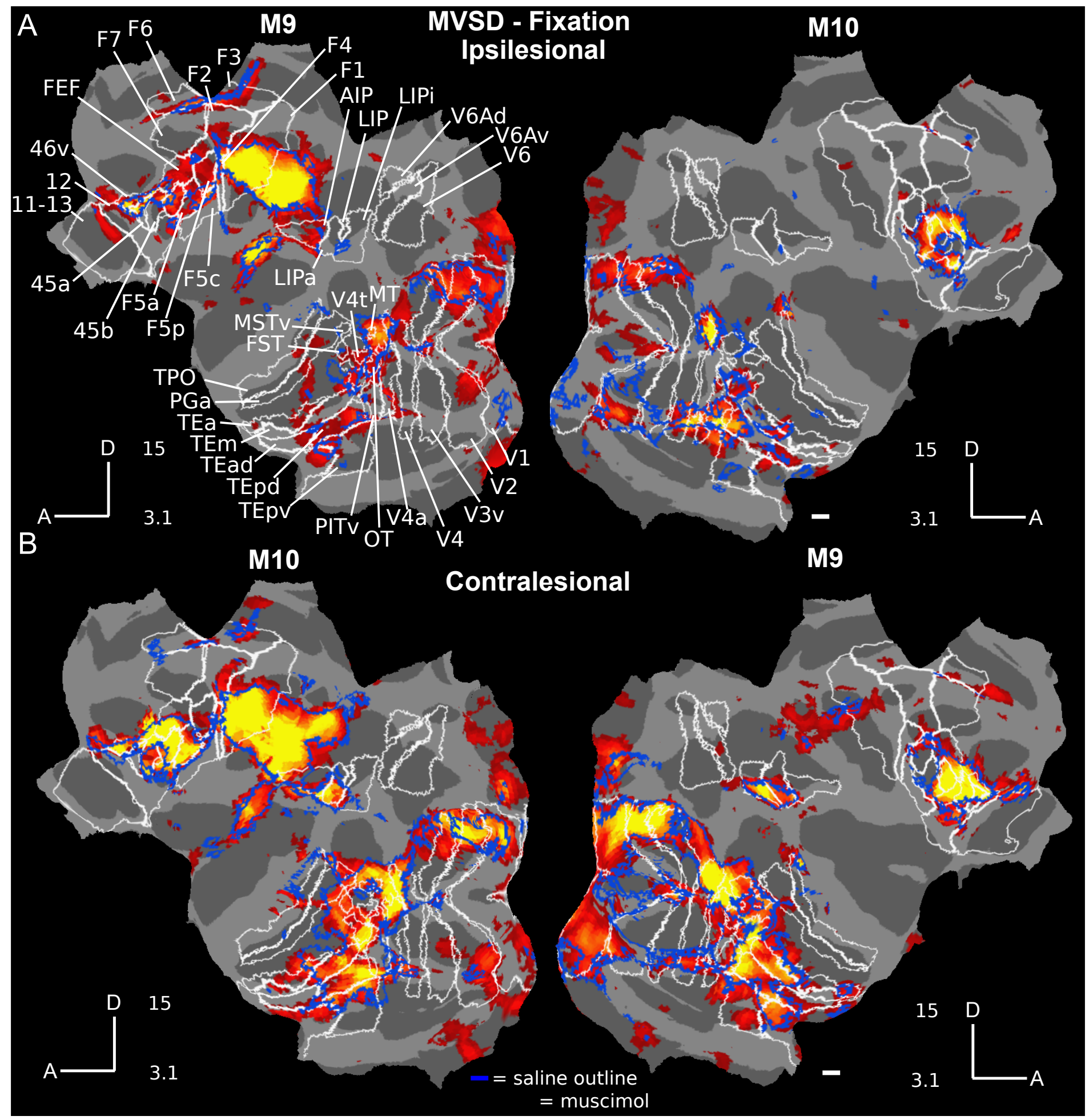


Fig 4 S0 - old Fig S1

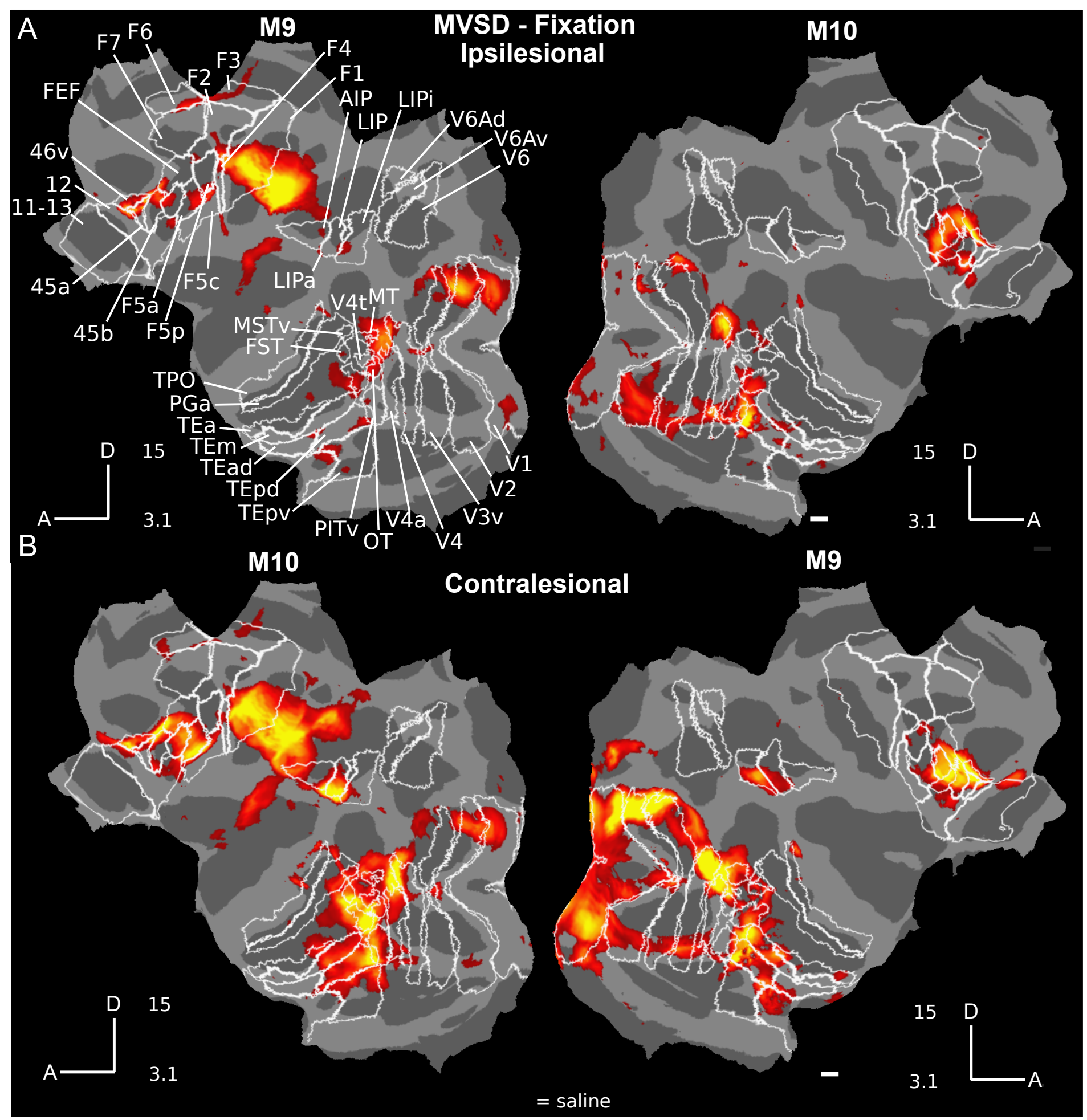


Fig. 4S1. RFX GLM

\section{M9 Saline}

Ipsilesional

CJ

DET

CJ vs. DET

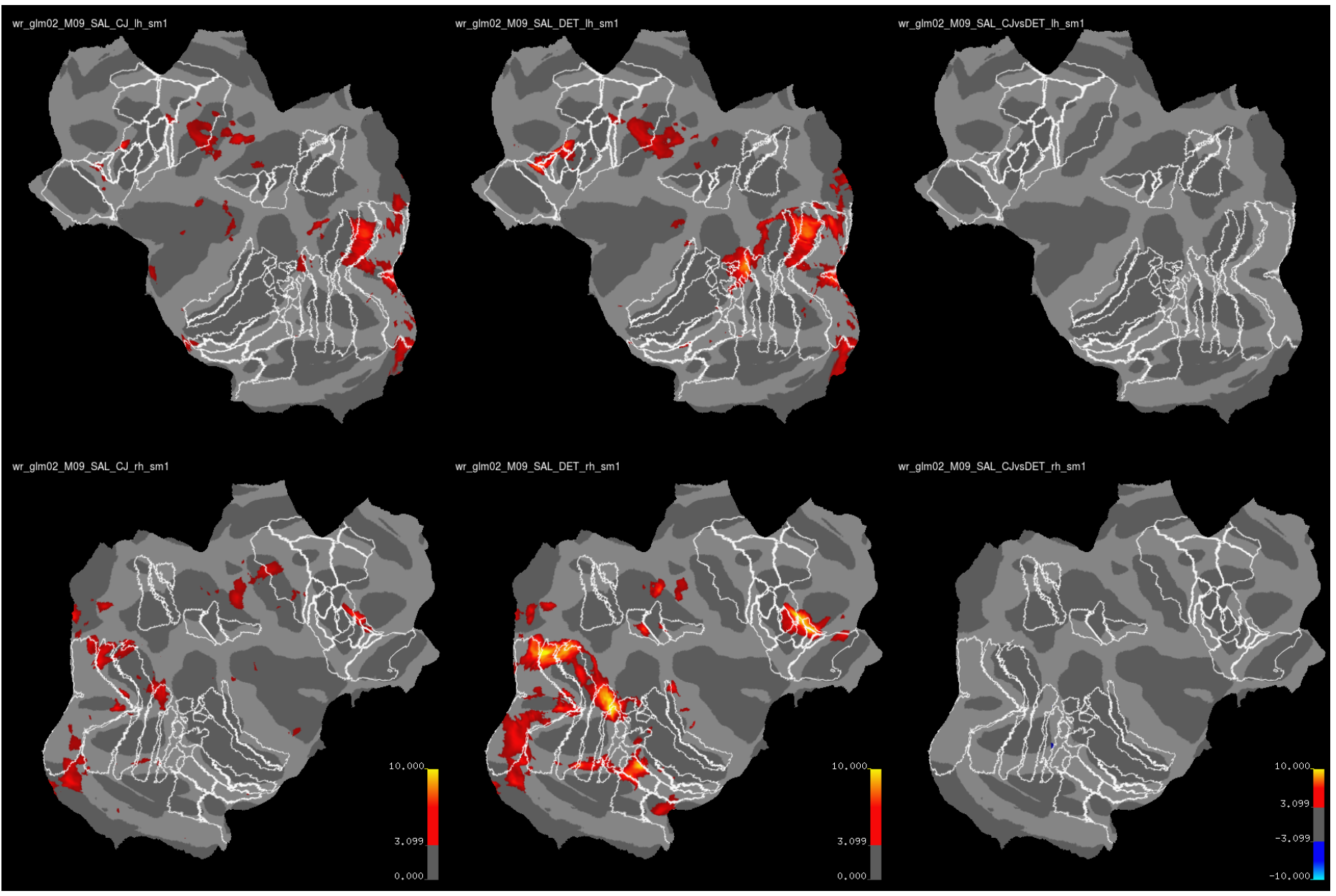

Contralesioanl 
Fig 4S2 RFX GLM

M9 Muscimol

Ipsilesional

$\begin{array}{lll}\text { CJ DET } & \text { CJ vs. DET }\end{array}$

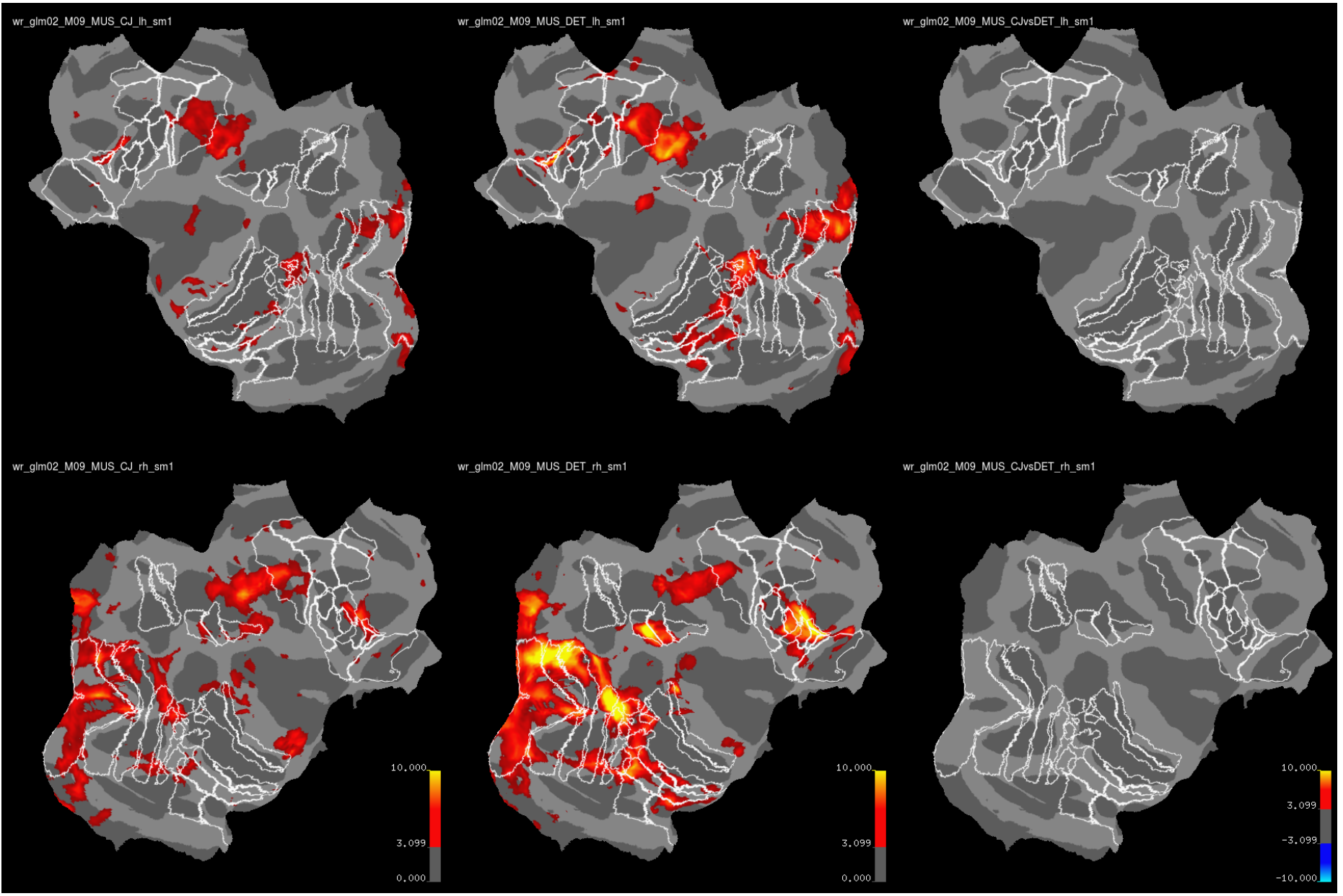

Contralesioanl 
Fig 4S3 RFX GLM

\section{M10 Saline}

\section{Contralesioanl}

CJ

DET

CJ vs. DET

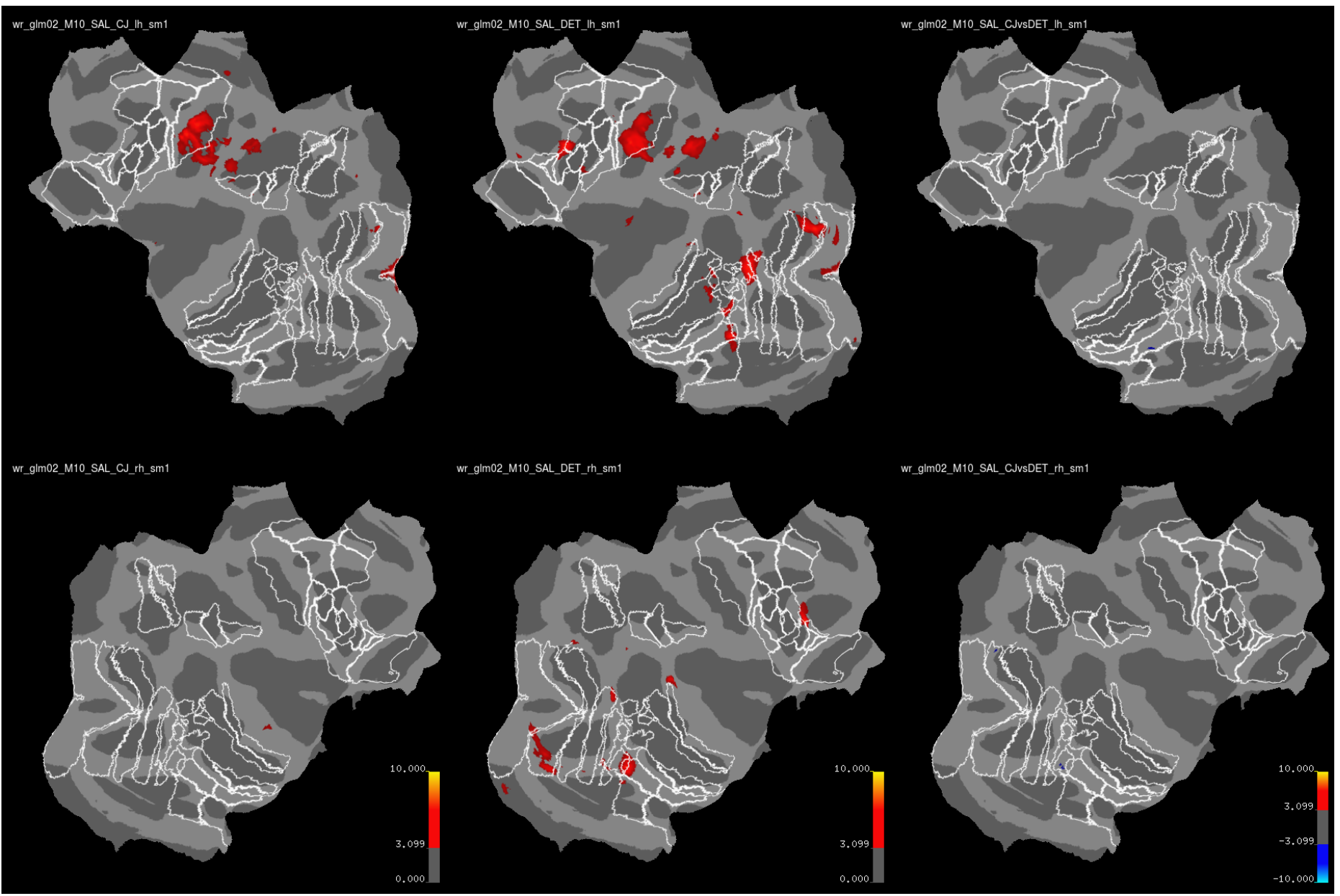

Ipsilesional 
Fig 4S4 RFX GLM

M10 Muscimol

Contralesioanl

CJ

DET

CJ vs. DET

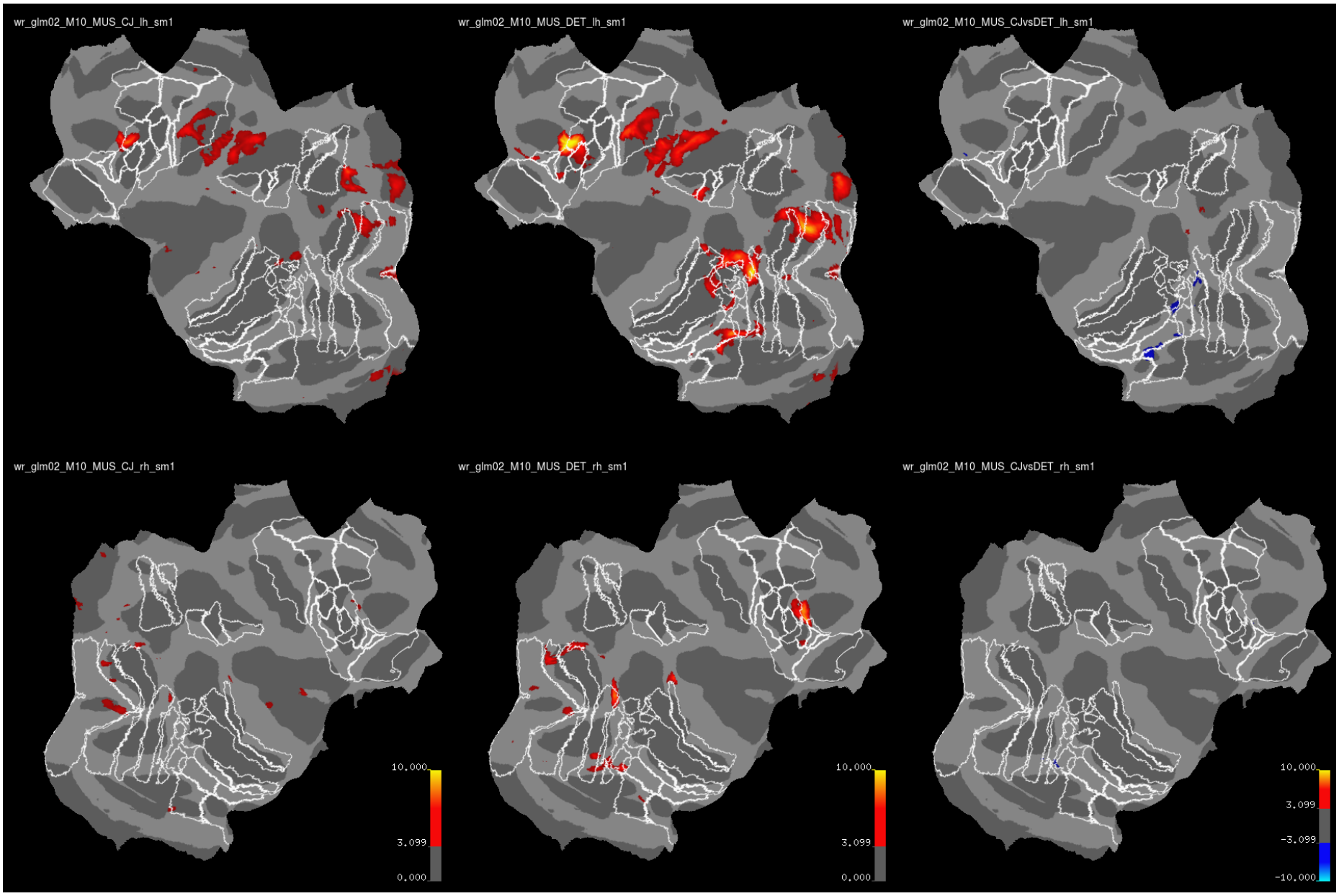

Ipsilesional 
Figure 4S5

Two sample T-test

Beta strength GLM Fixation-Muscimol

vs.

Beta strength GLM Fixation-Saline

Ipsilesional

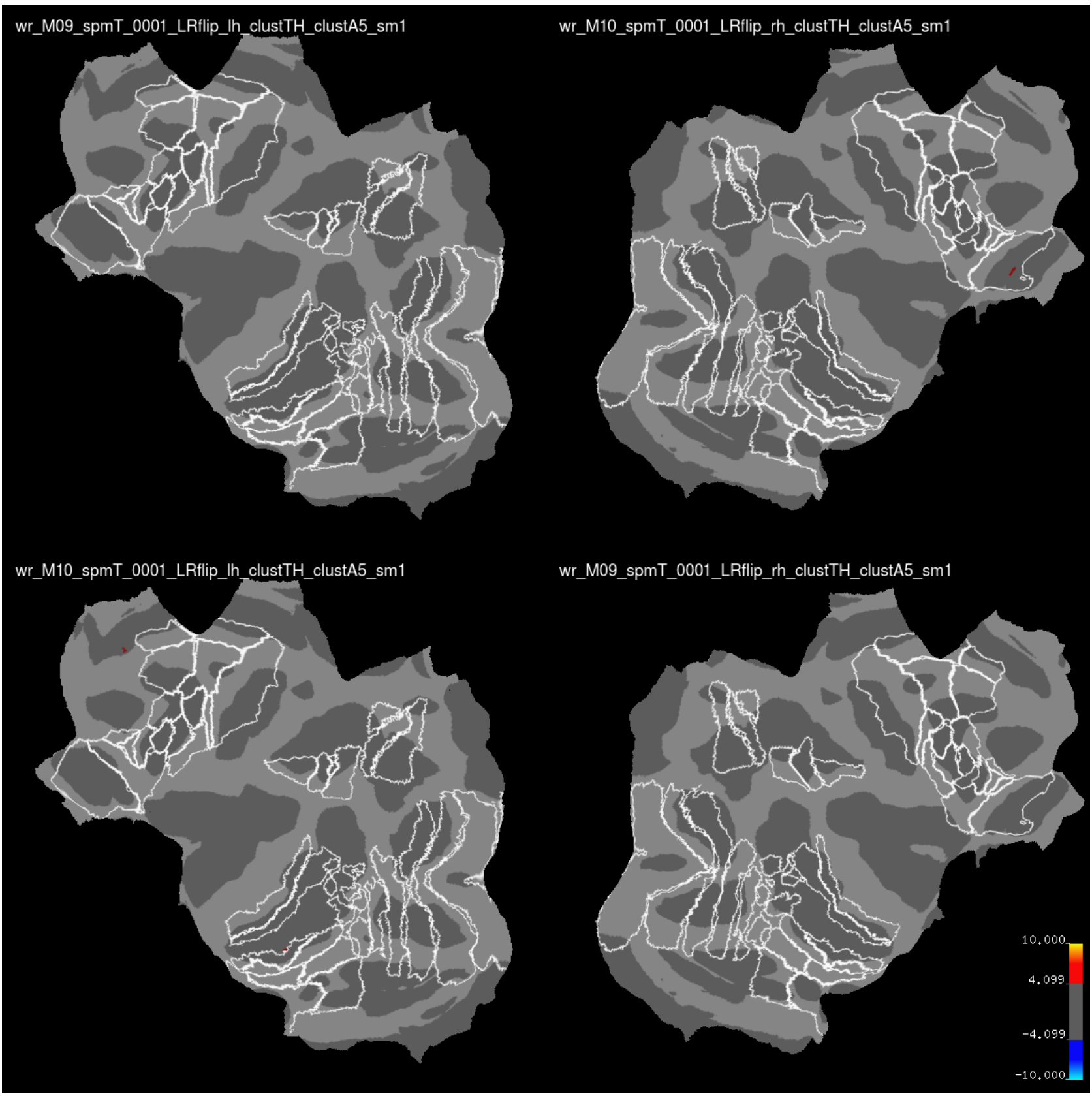

Contralesional 
Fig 4S6 Effect of RT-variability regressor on GLM RFX SAL for M9

\section{Ipsilesional}

(A) GLM RFX with RT-variability regressor

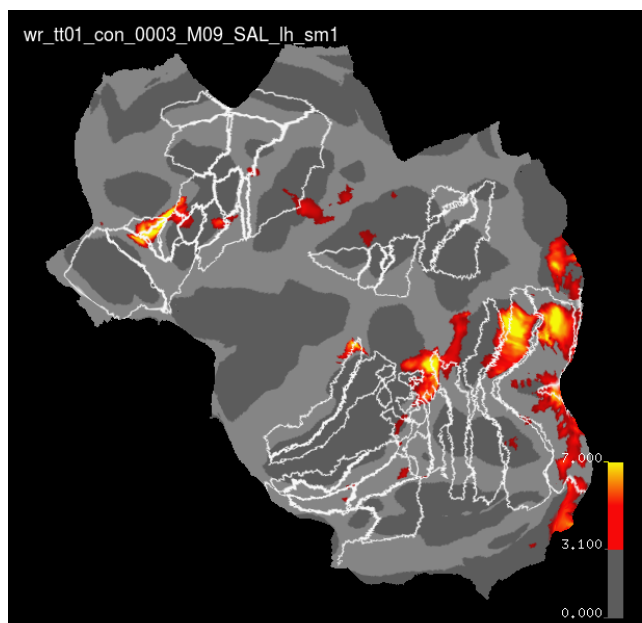

Wr_tt01_con_0003_M09_SAL_rh_sm1

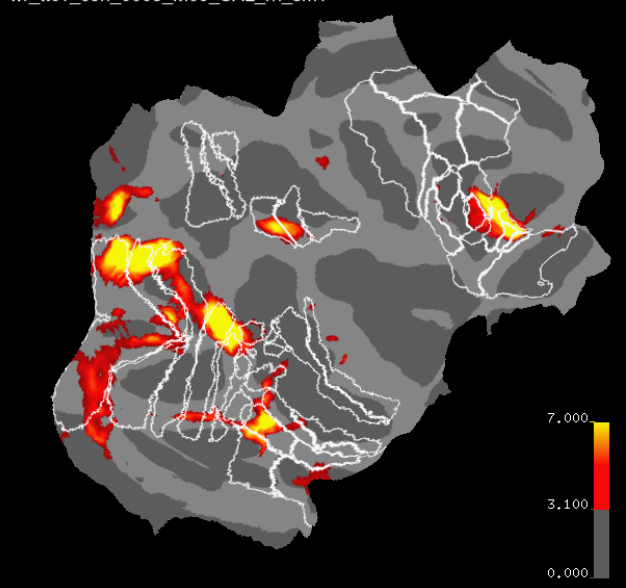

(B) GLM RFX without RT-variability regressor contrast (A)vs.(B)
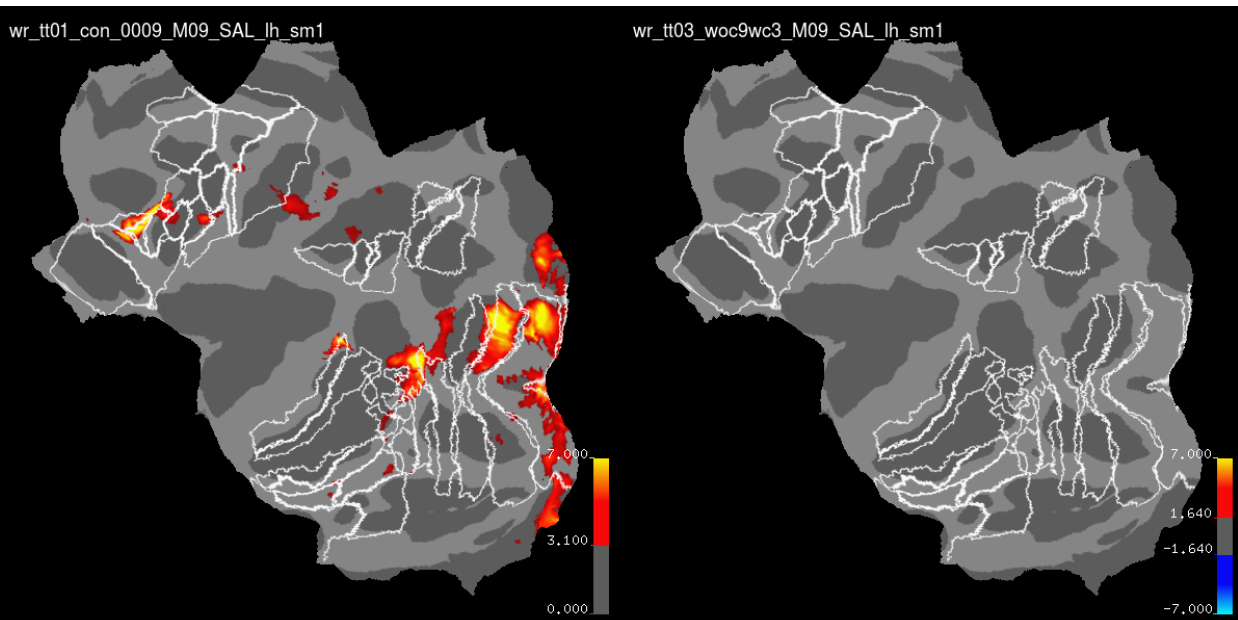

wr_tt01_con_0009_M09_SAL_rh_sm1

Wr_tt03_woc9wc3_M09_SAL_rh_sm1

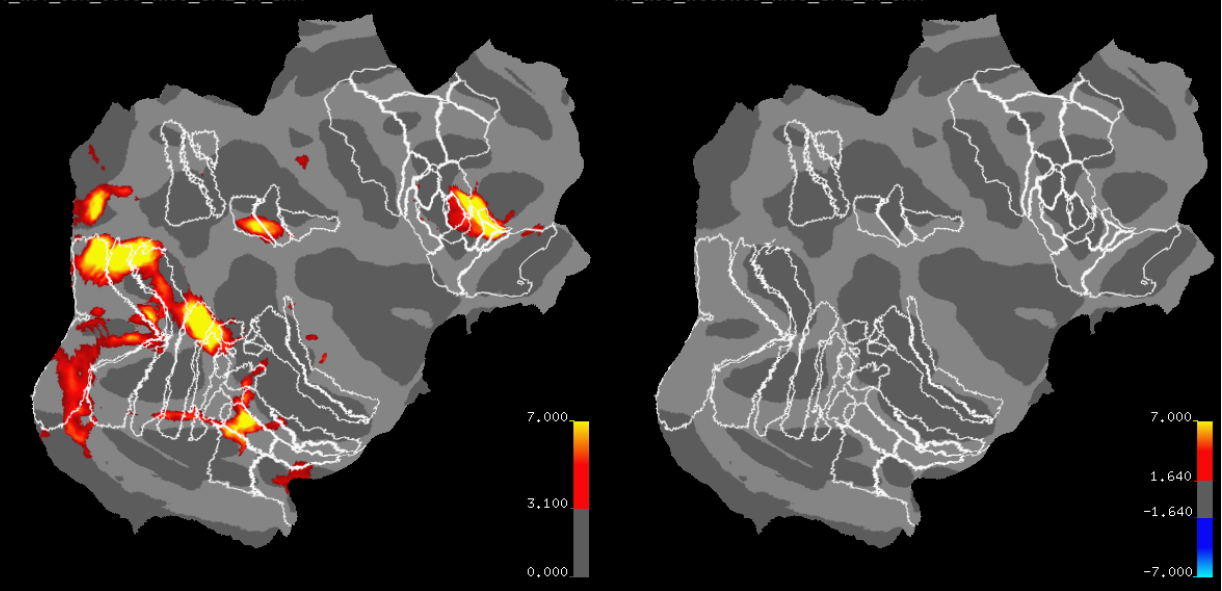

Contralesional 
Fig 4S7 Effect of RT-variability regressor on GLM RFX MUS for M9

\section{Ipsilesional}

(A) GLM RFX with RT-variability regressor

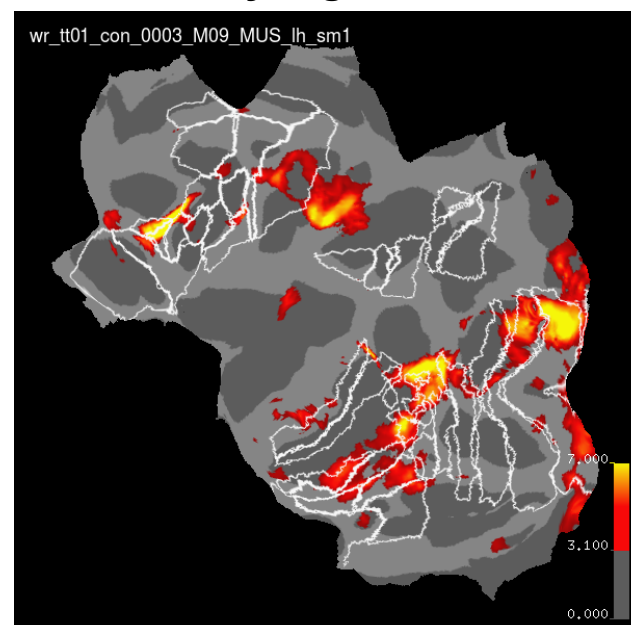

Wr_tt01_con_0003_M09_MUS_rh_sm1

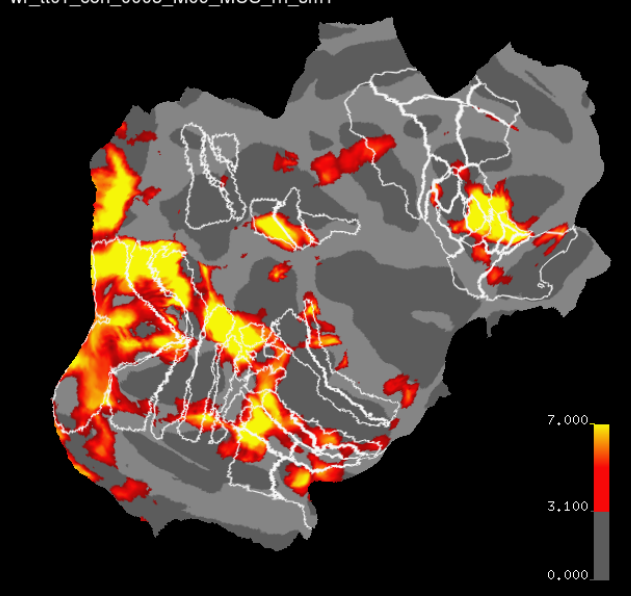

(B) GLM RFX without

contrast (A)vs.(B)

\section{RT-variability regressor}

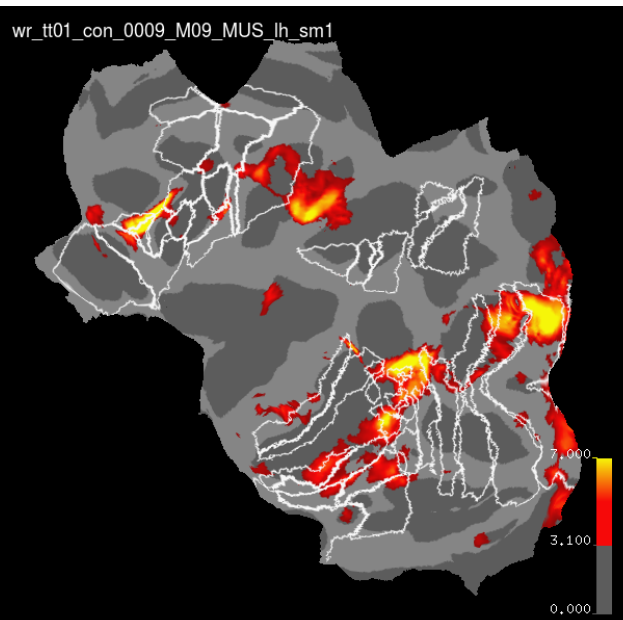

Wr_tt01_con_0009_M09_MUS_rh_sm1

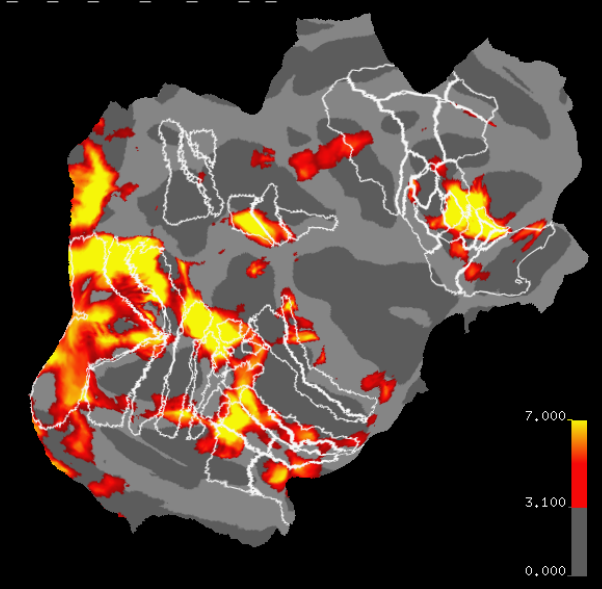

Wr_tt03_woc9w03_M09_MUS_rh_sm1

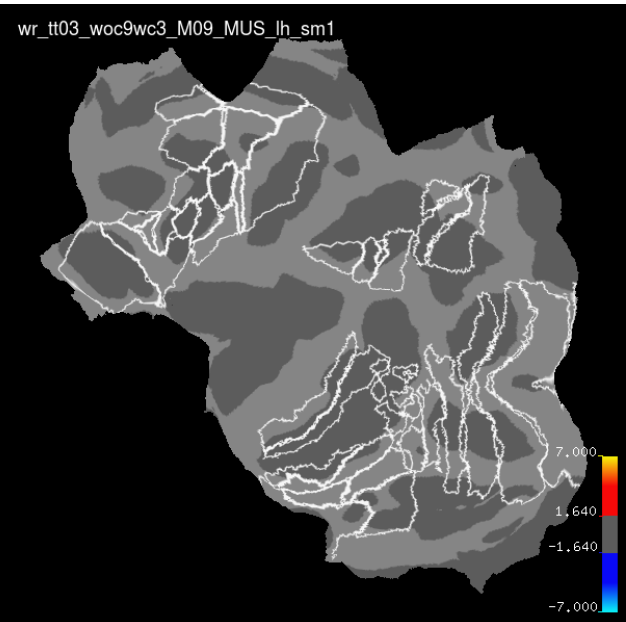

\section{Contralesional}


Fig 4S8 Effect of RT-variability regressor on GLM RFX SAL for M10

\section{Ipsilesional}

(A) GLM RFX with RT-variability regressor

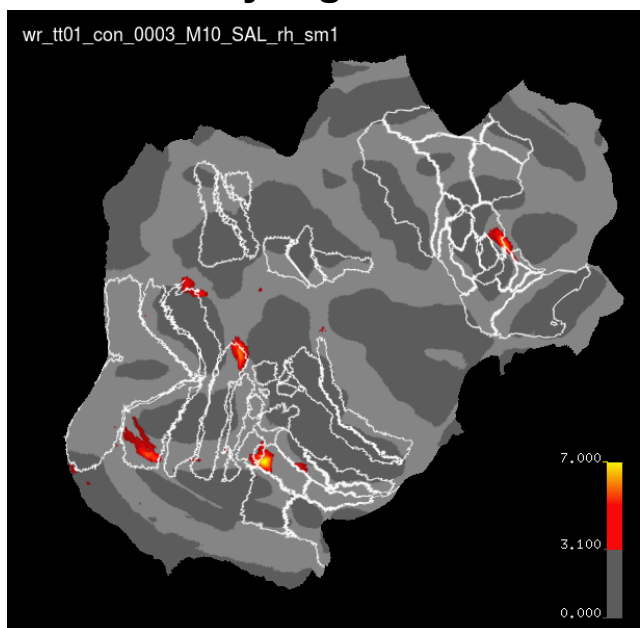

Wr_tt01_con_0003_M10_SAL_lh_sm1

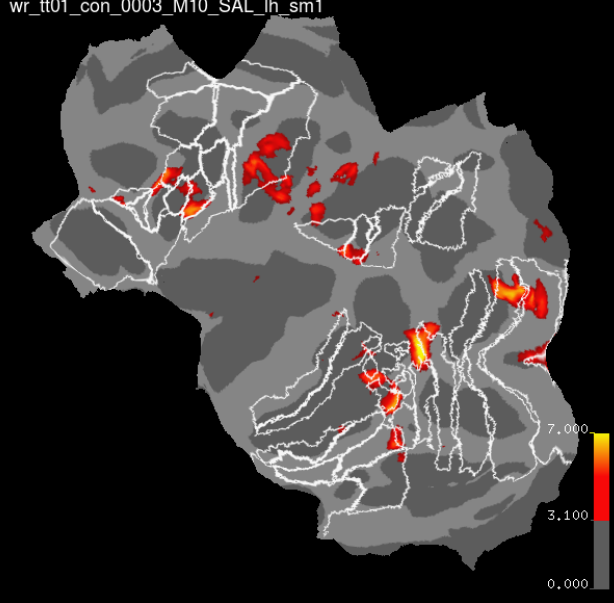

(B) GLM RFX without

\section{RT-variability regressor}

wr_tt01_con_0009_M10_SAL_rh_sm1

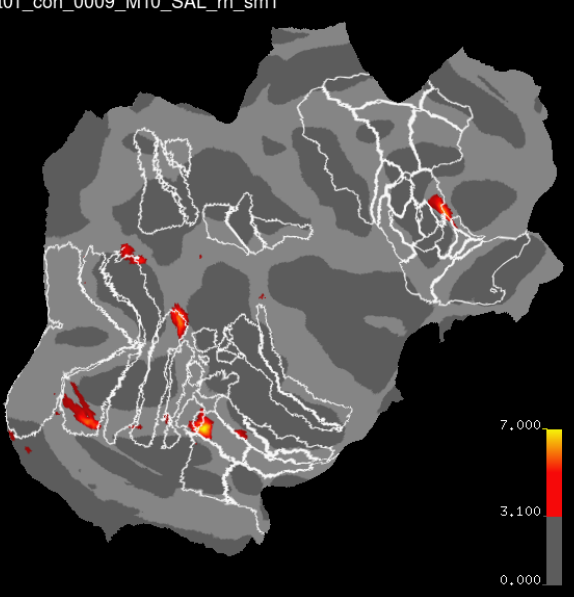

wr_tt03_woc9wc3_M10_SAL_rh_sm1

wr_tt01_con_0009_M10_SAL_lh_sm1

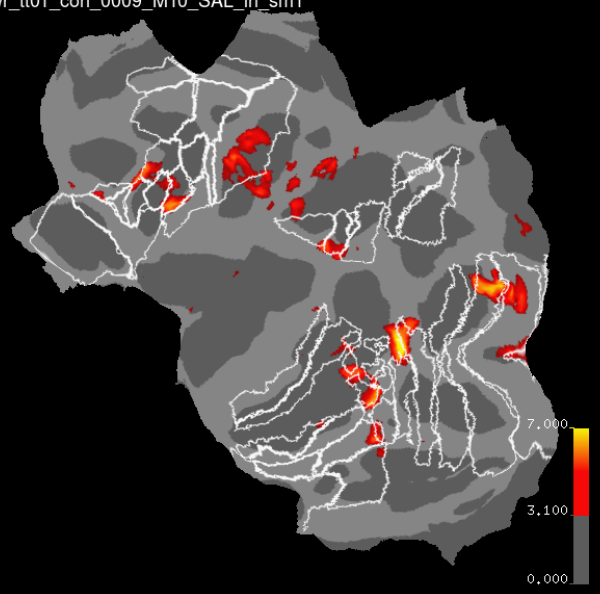

wr_tt03_woc9wc3_M10_SAL_lh_sm1

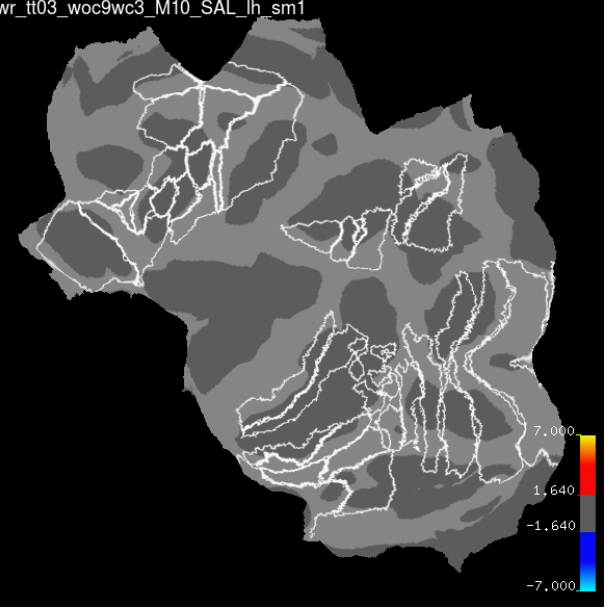

contrast (A)vs.(B)

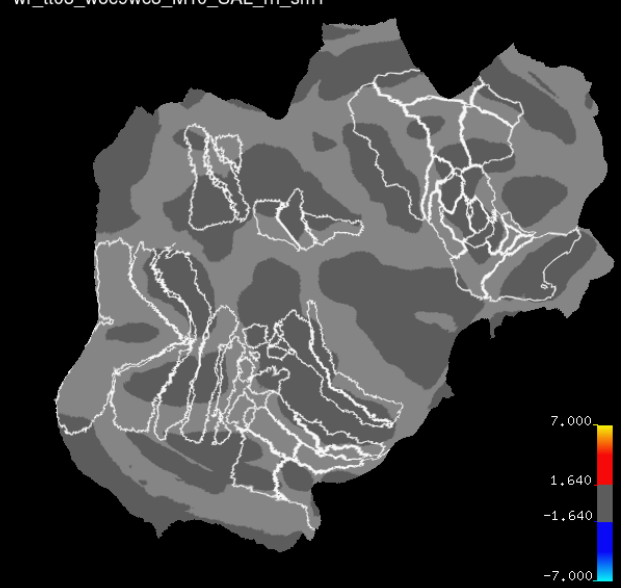

Contralesional 
Fig 4S9 Effect of RT-variability regressor on GLM RFX MUS for M10

\section{Ipsilesional}

(A) GLM RFX with RT-variability regressor

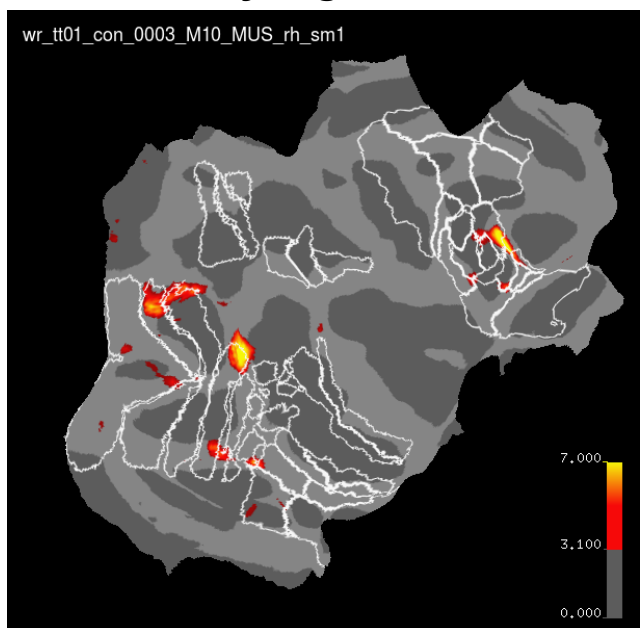

wr_tt01_con_0003_M10_MUS_lh_sm1

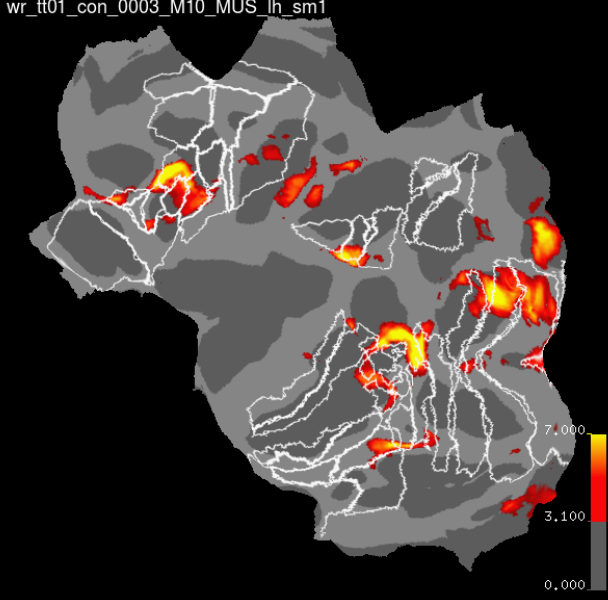

(B) GLM RFX without

contrast (A)vs.(B)

\section{RT-variability regressor}

wr_tt01_con_0009_M10_MUS_rh_sm1

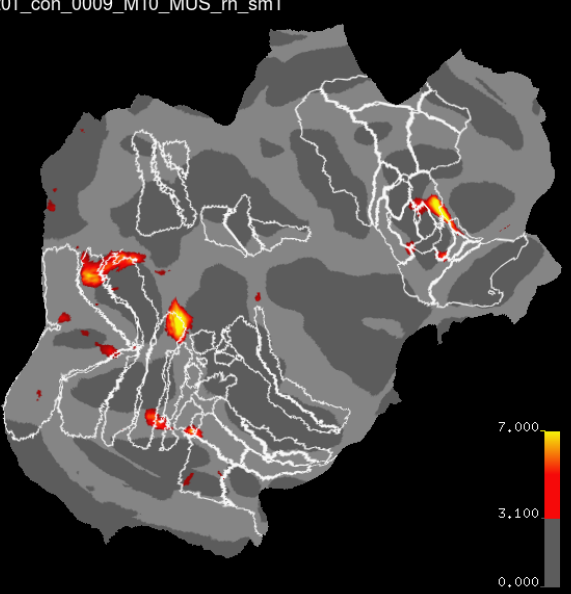

Wr_tt01_con_0009_M10_MUS_lh_sm1

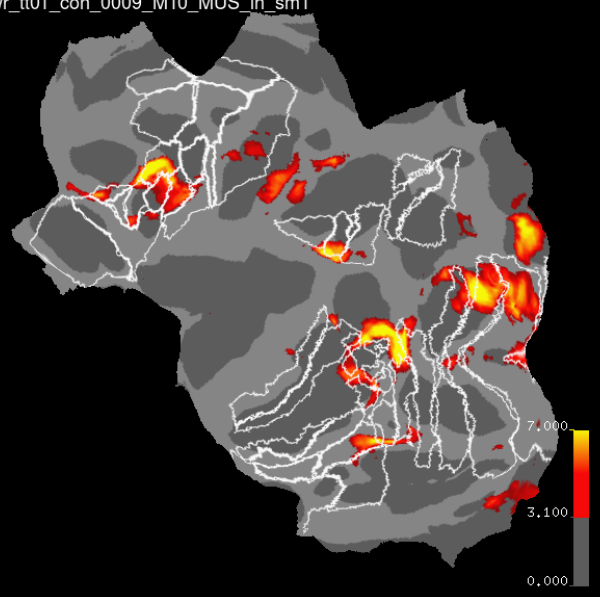

wr_tt03_woc9wc3_M10_MUS_lh_sm1

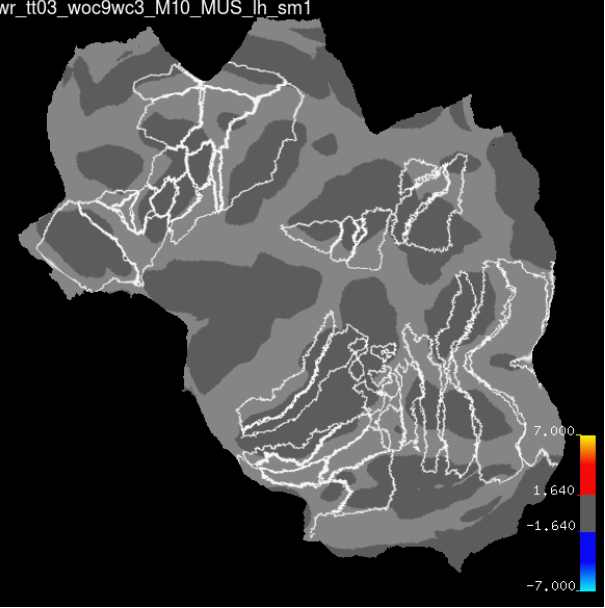

Wr_tt03_woc9wc3 M10_MUS_rh_sm1

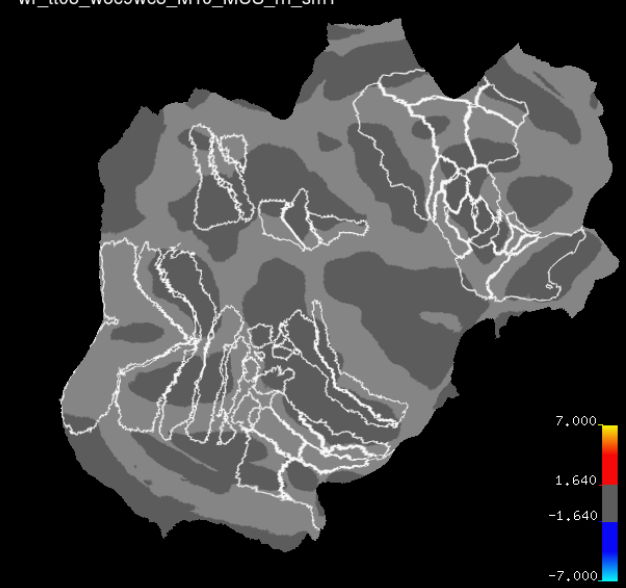

$-7.000$

Contralesional 
Fig 5 S0 - old Fig S2 renamed

GLM RFX T-test (Saline vs. Muscimol)
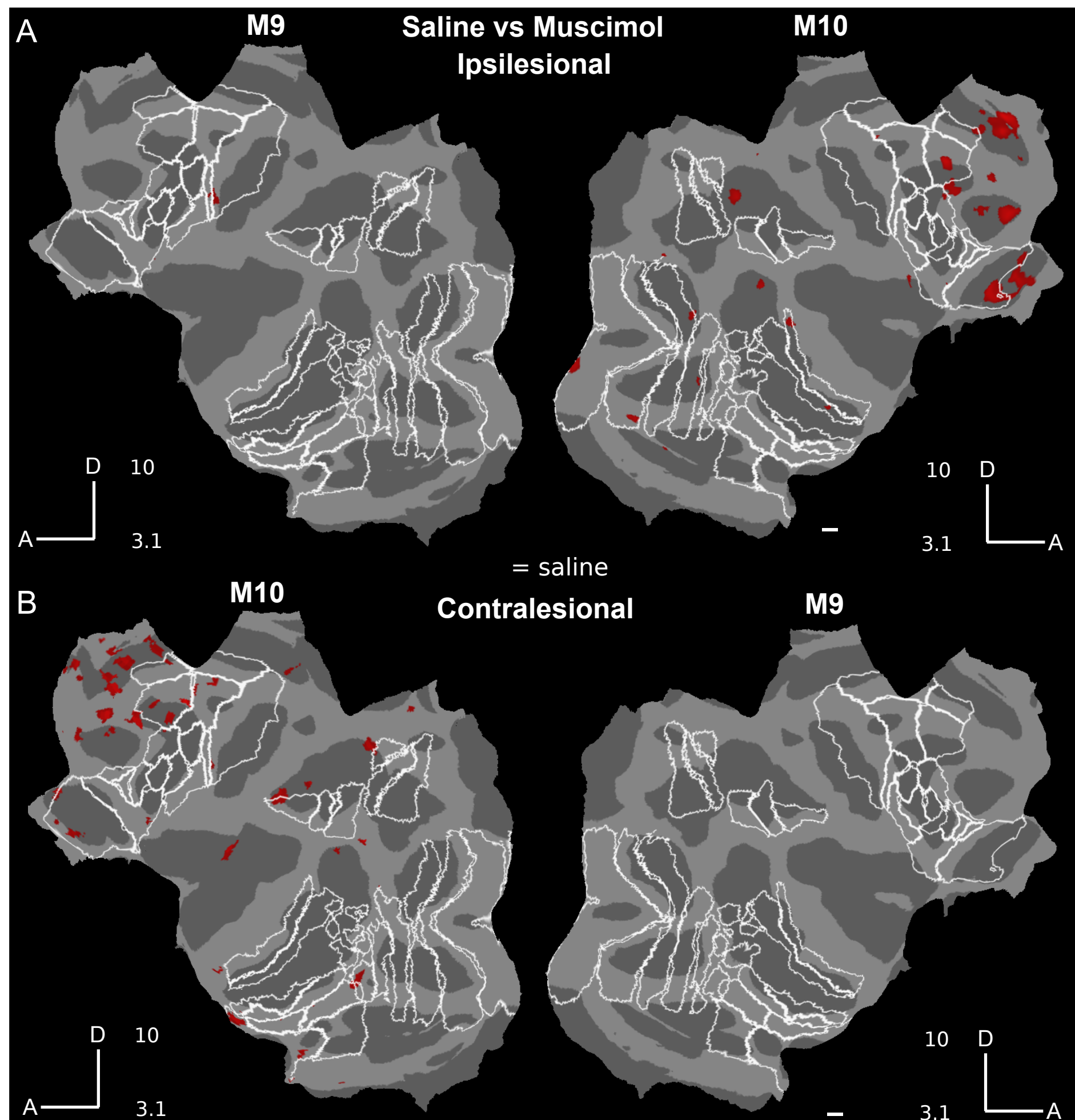

alesional

\section{M9}

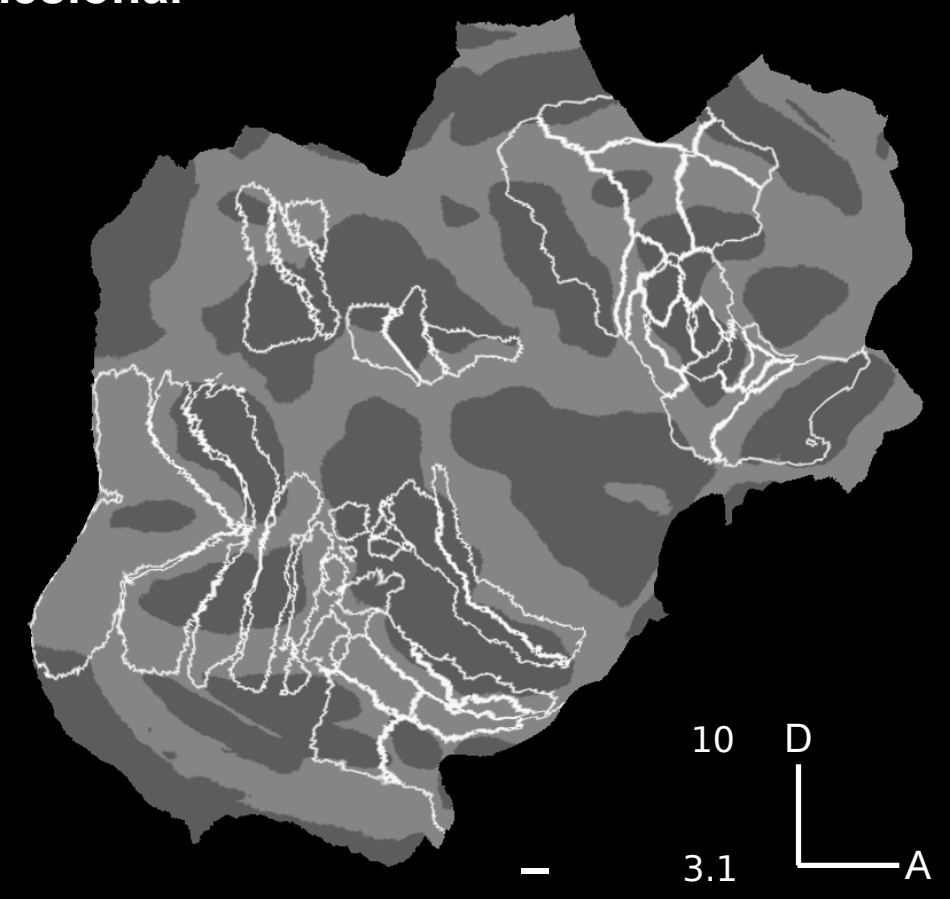


Figure 5S1. RFX GLM, two sample T-test Muscimol vs. Saline Ipsilesional

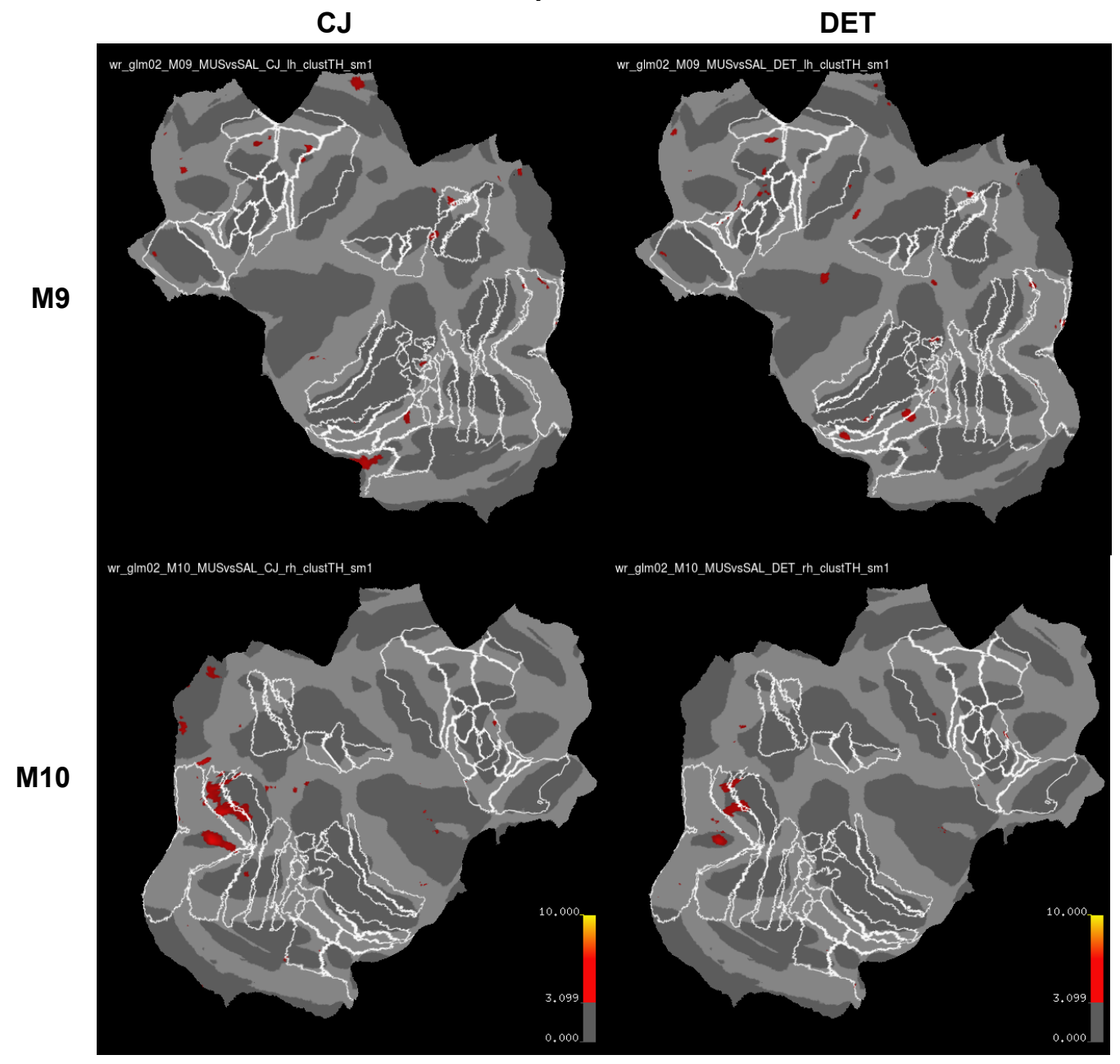

\section{Contralesional}

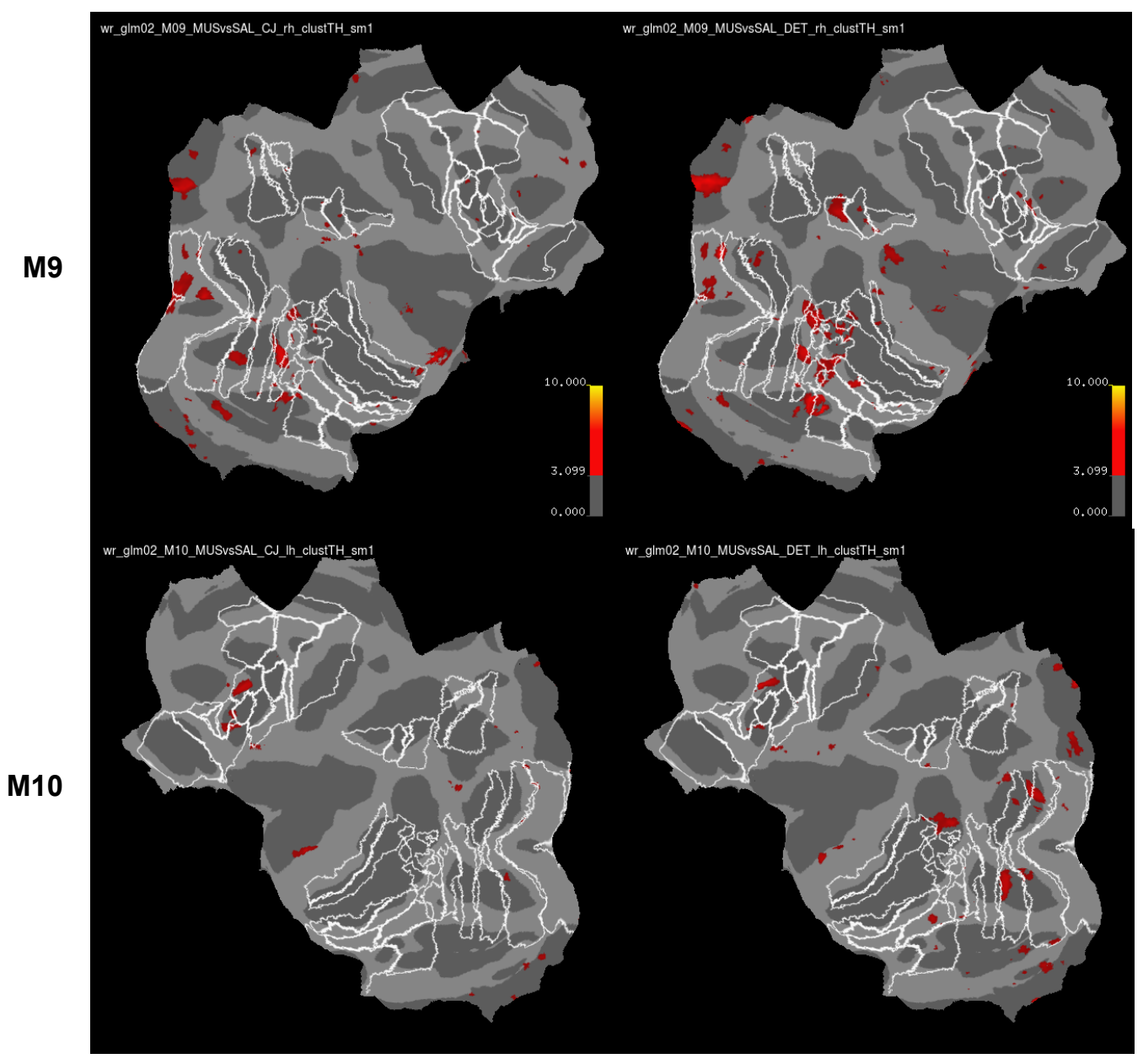


Fig 5 S2 Effect of RT-variability regressor on GLM RFX contrast MUS vs. SAL for M9

\title{
Ipsilesional
}

GLM RFX with

RT-variability regressor

\author{
GLM RFX without \\ RT-variability regressor
}
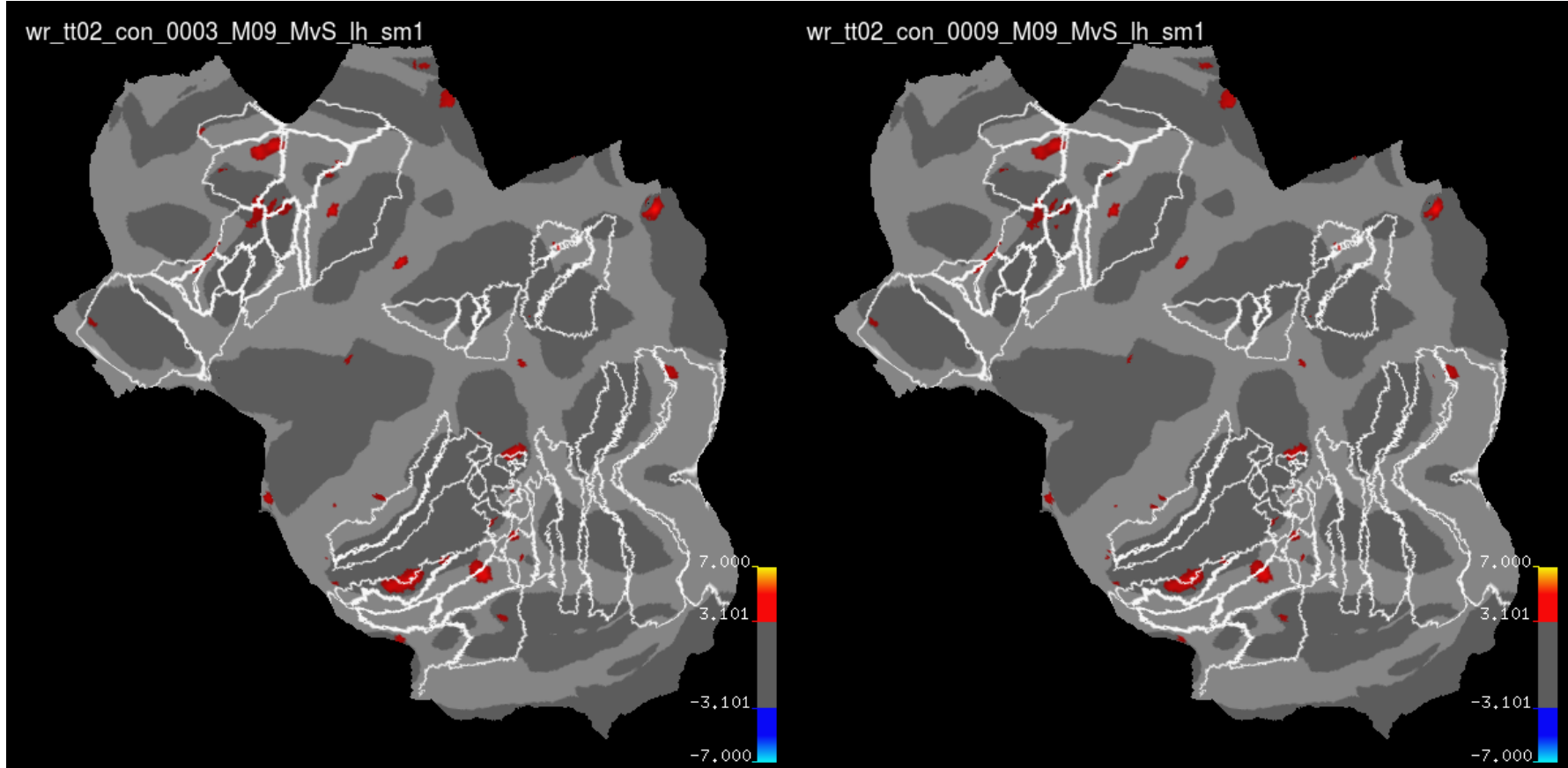

wr_tt02_con_0003_M09_MvS_rh_sm1

Wr_tt02_con_0009_M09_MvS_rh_sm1

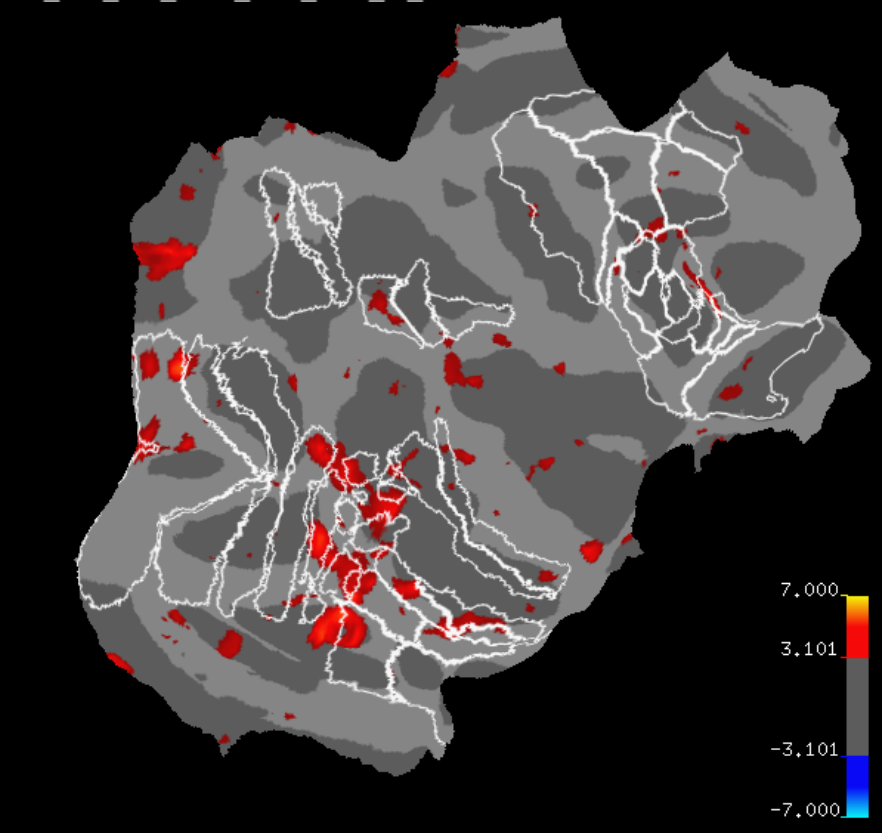

\section{Contralesional}


Fig 5 S3 Effect of RT-variability regressor on GLM RFX contrast MUS vs. SAL for M10

\section{GLM RFX with}

RT-variability regressor
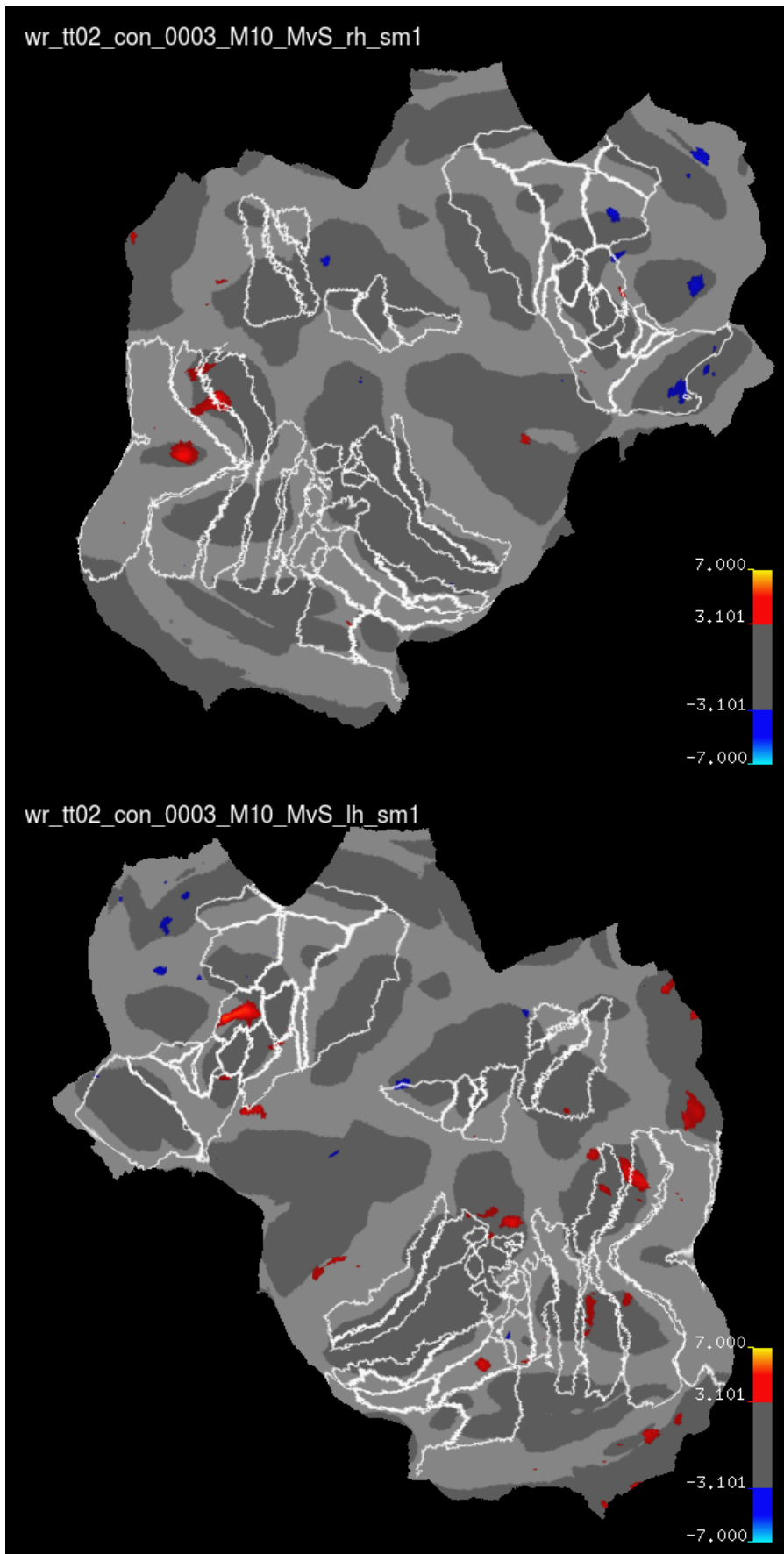

\section{Ipsilesional}

\author{
GLM RFX without
}

RT-variability regressor

Wr_tt02_con_0009_M10_MvS_rh_sm1

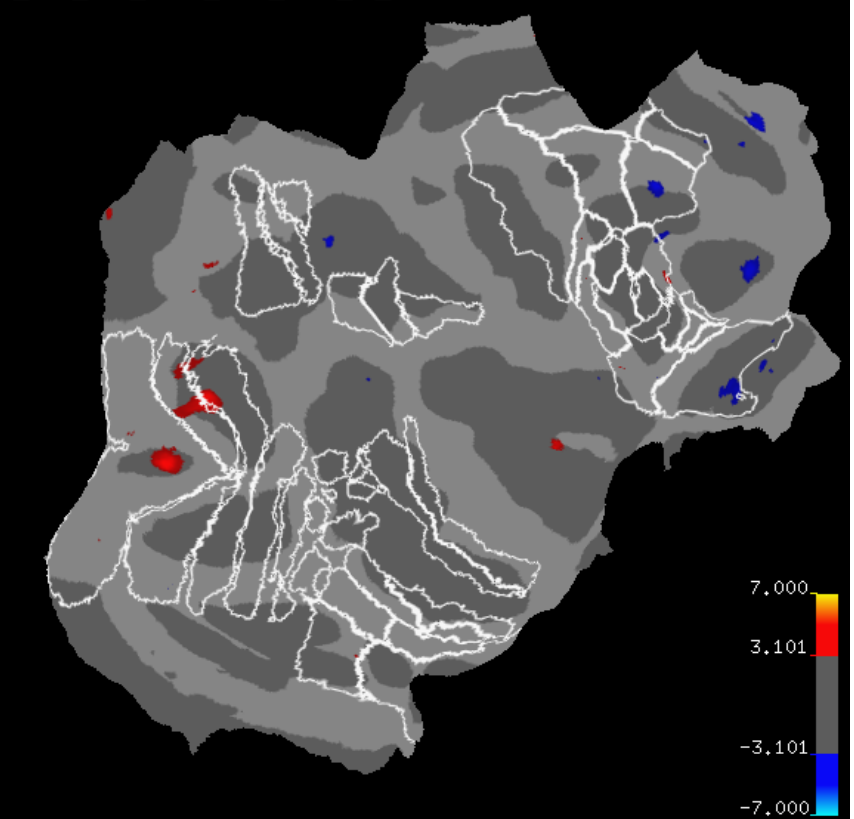

wr_tt02_con_0009_M10_MvS_lh_sm1

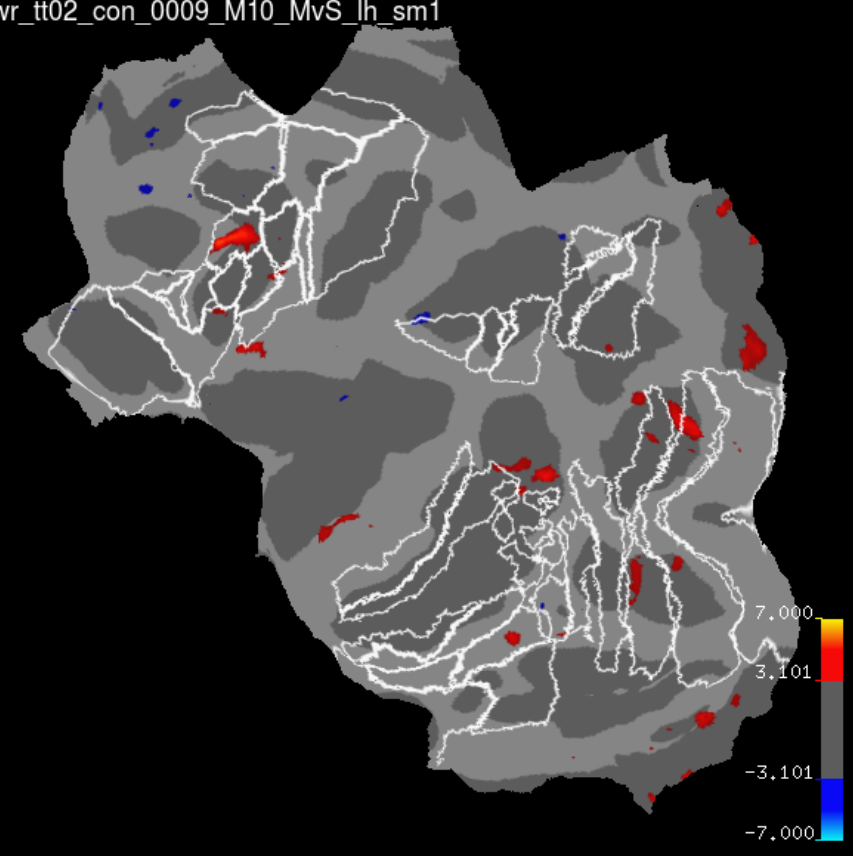

\section{Contralesional}


Fig 5

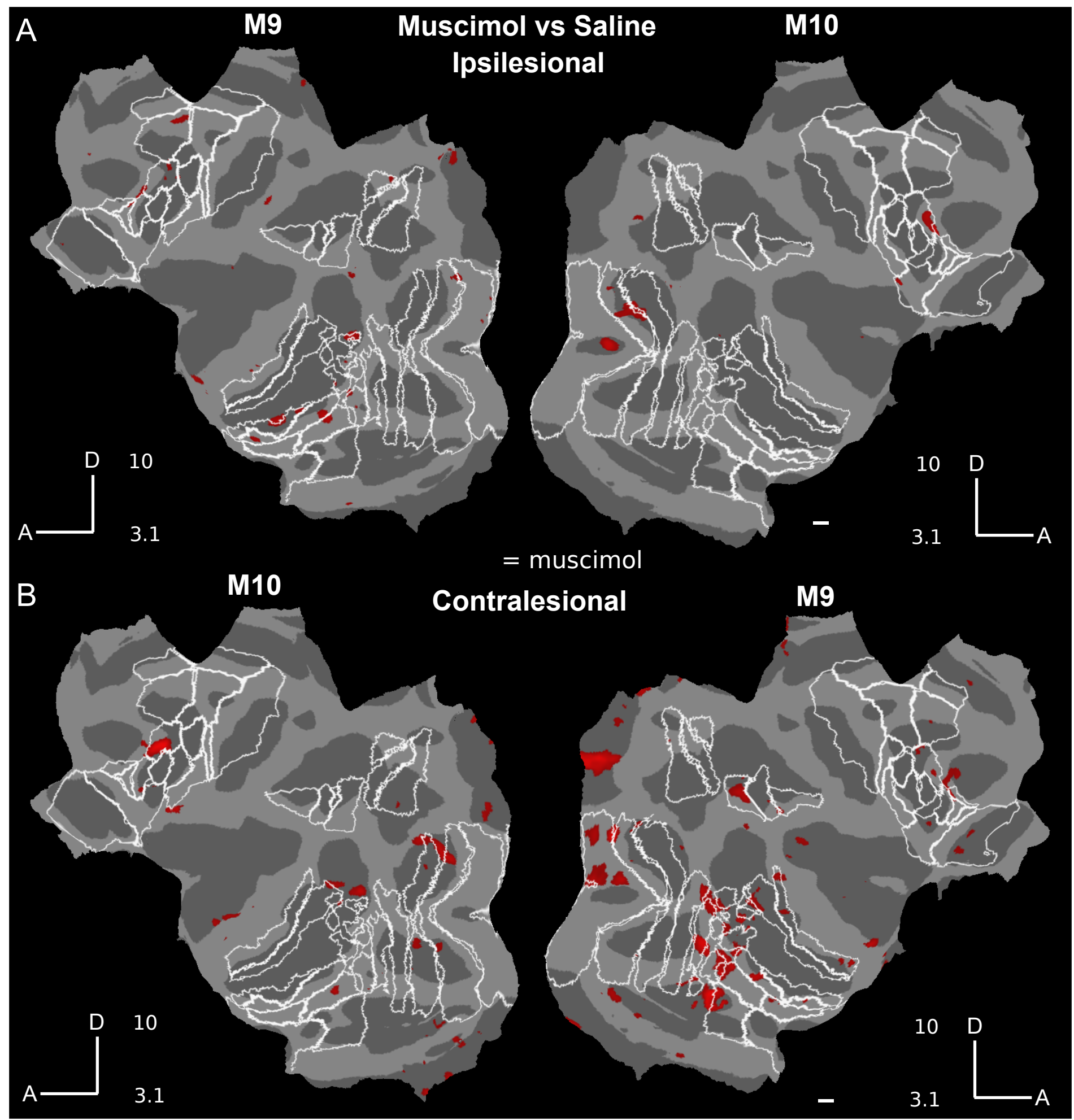


Figure 6

A

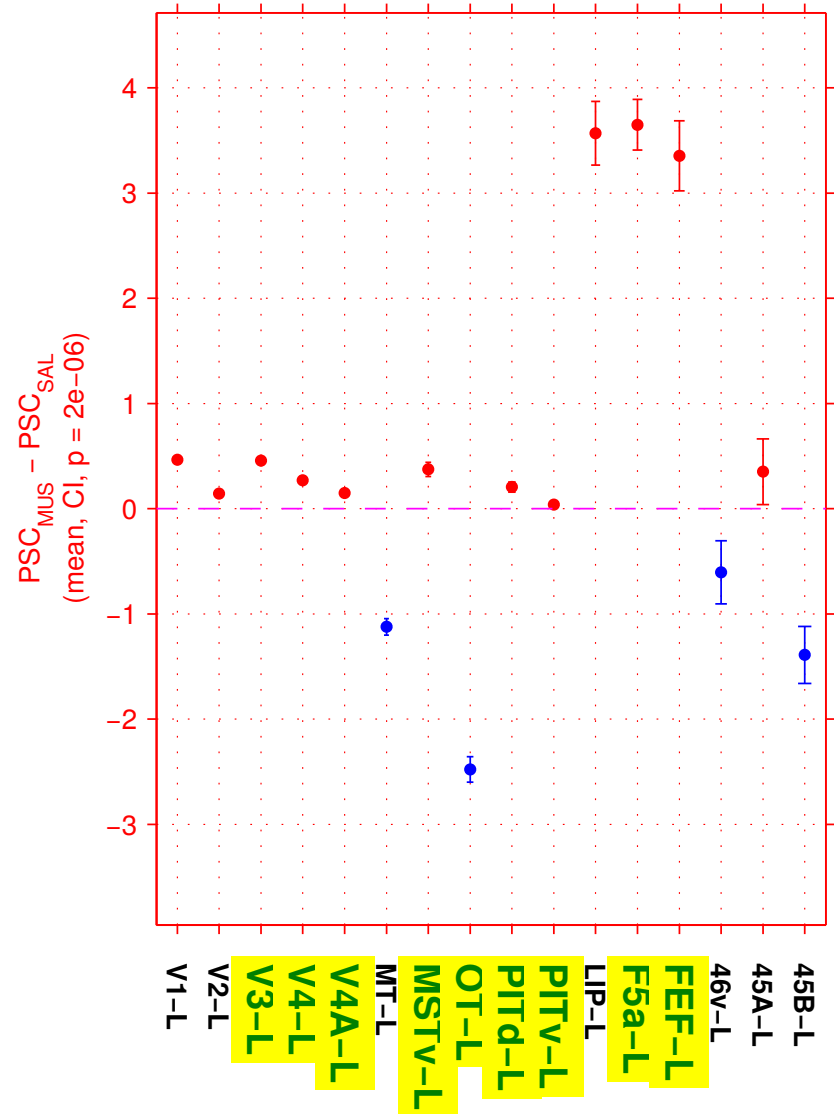

B

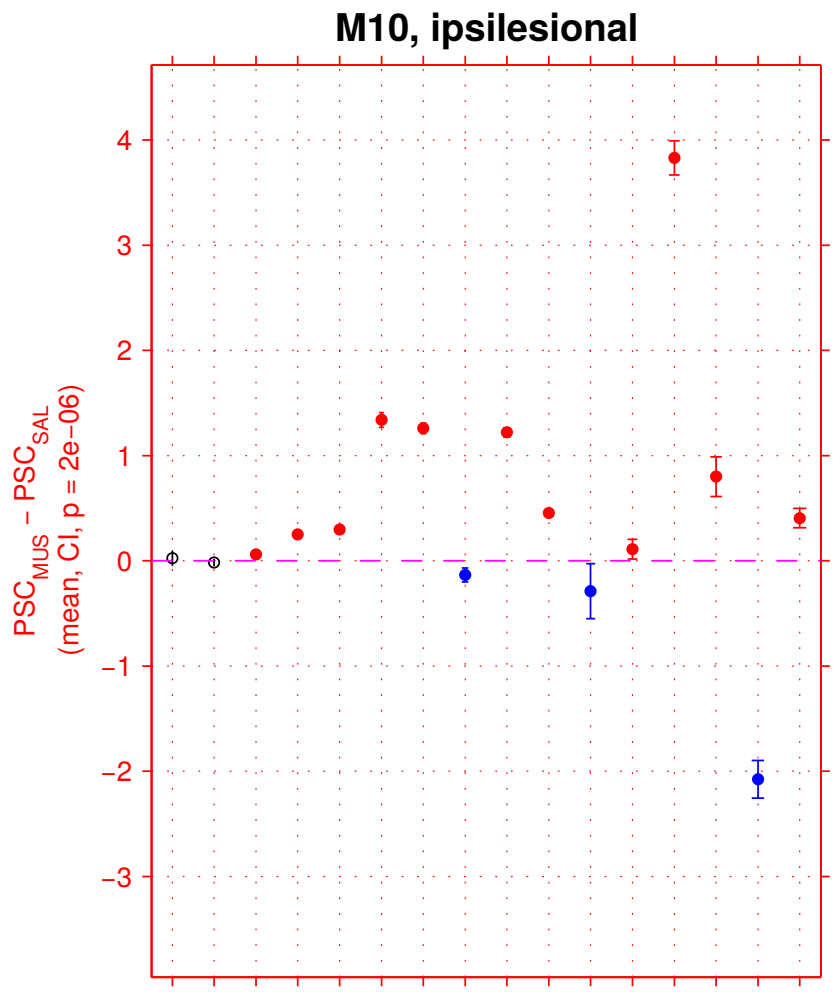

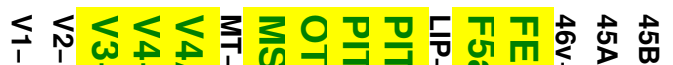

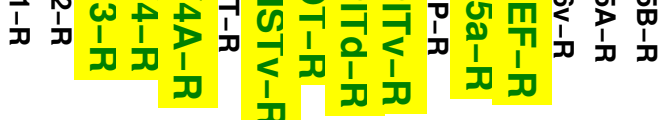

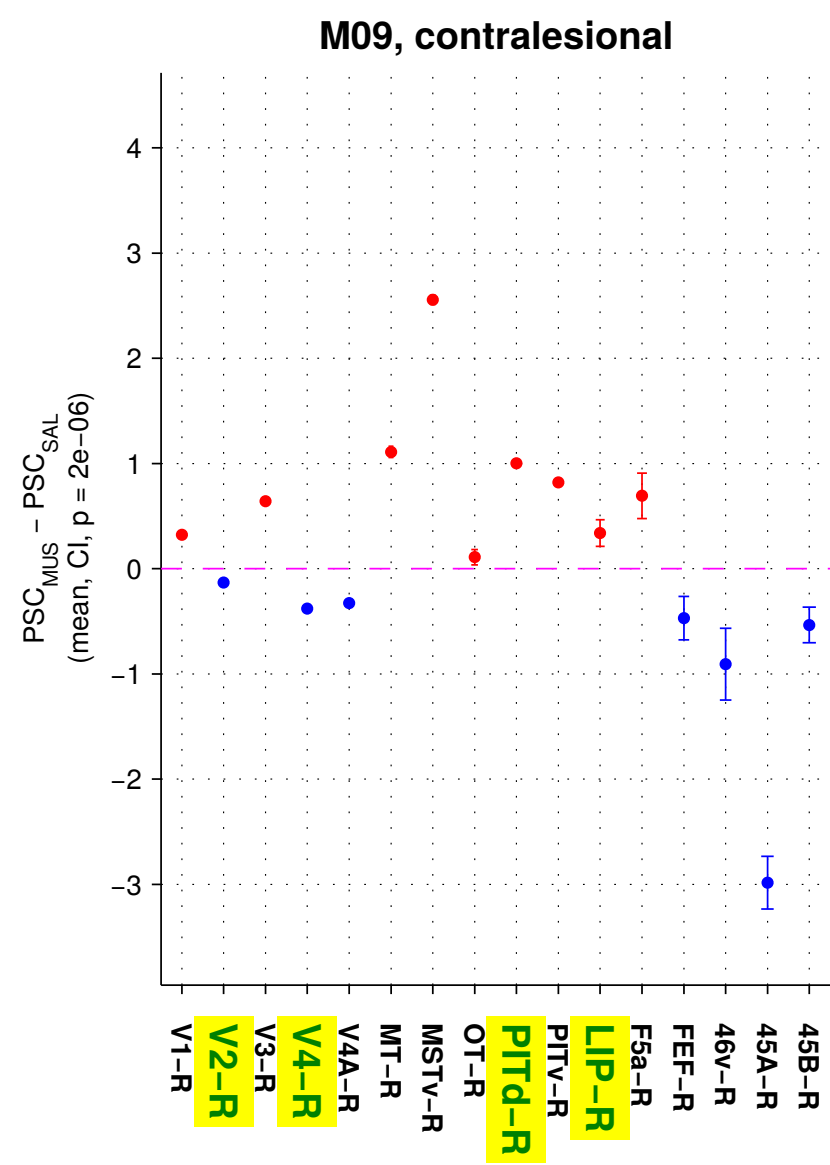

M10, contralesional

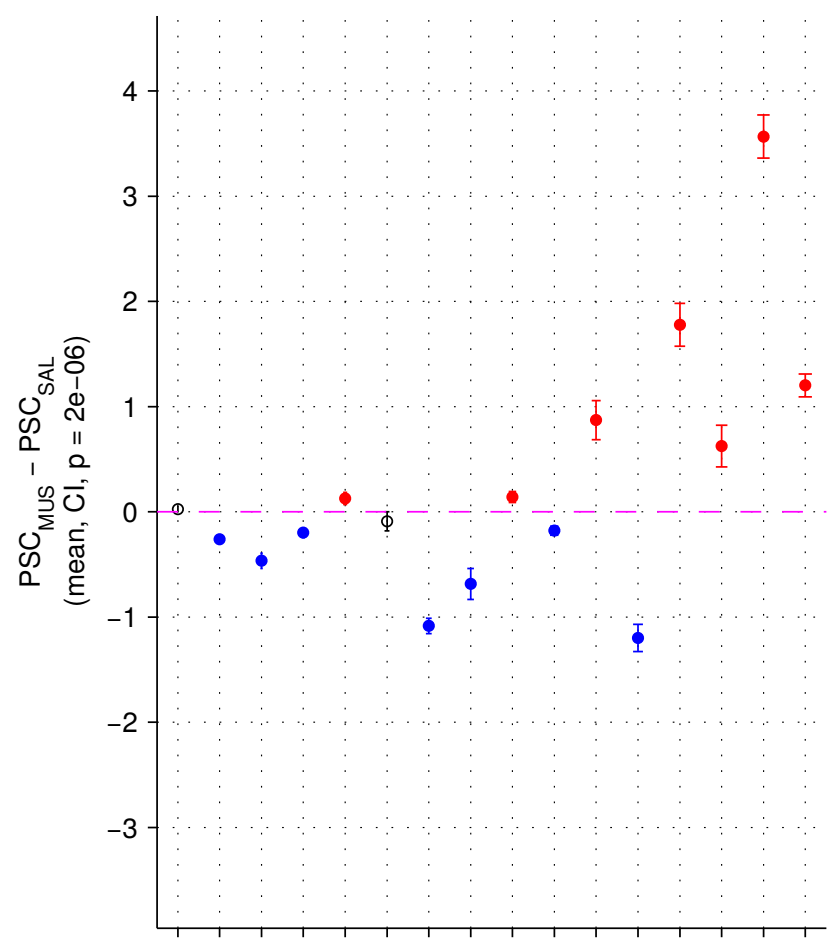

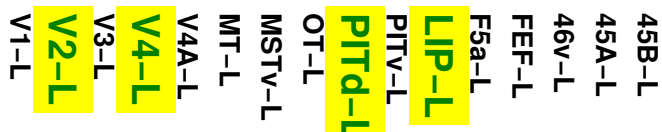


Fig 7
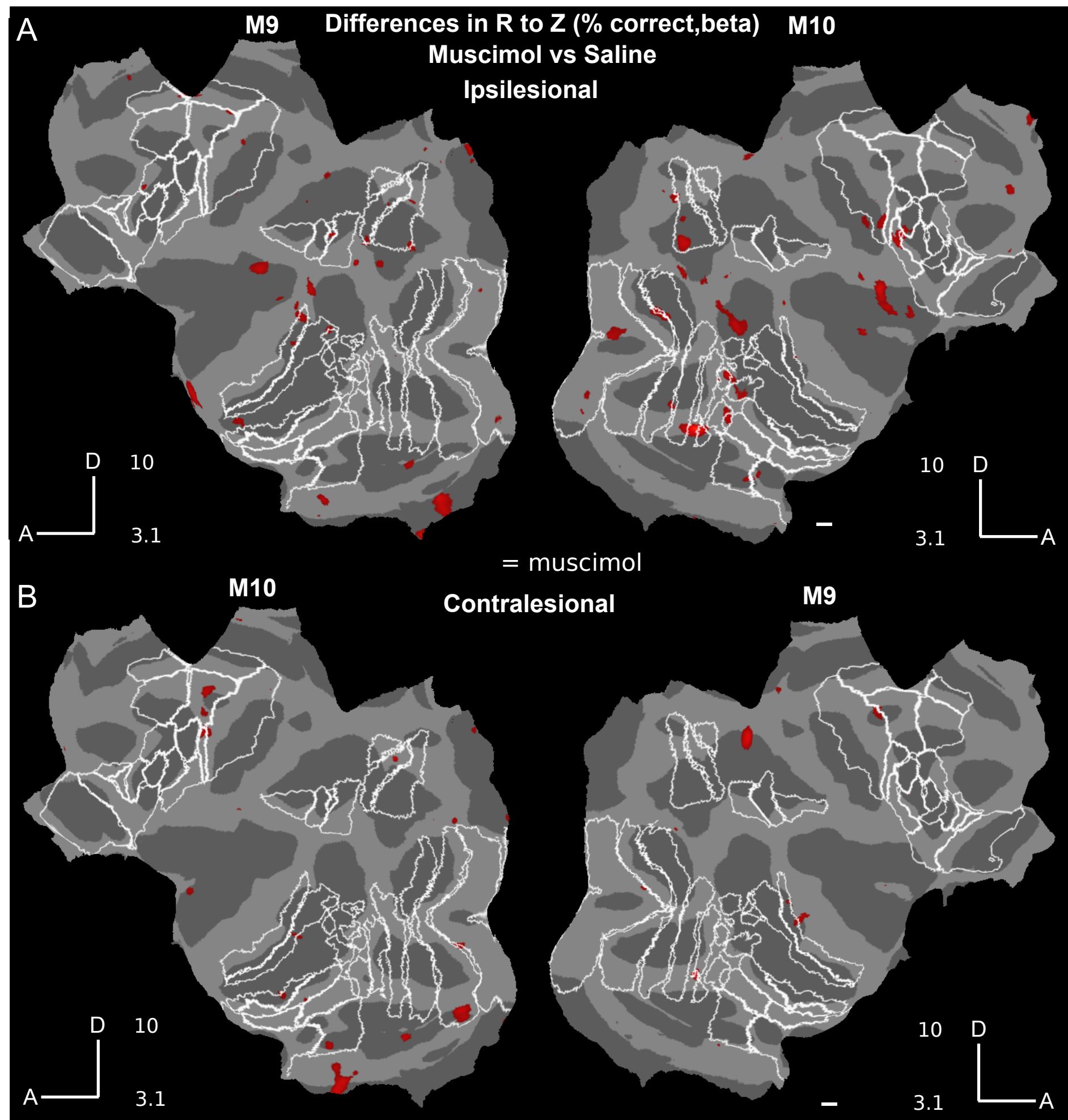
Figure 7S1. Behavioral correlations for saline and muscimol, and the difference between muscimol and saline for M9

\section{Ipsilesional}
Saline
Muscimol
Muscimol vs. Saline
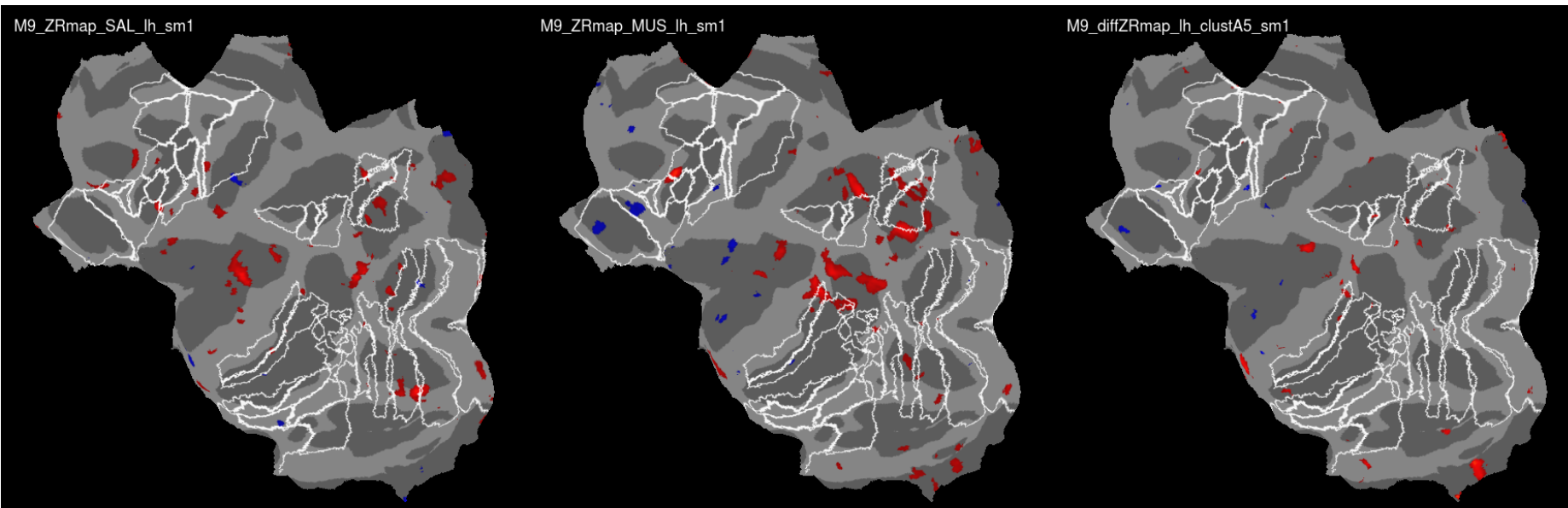

M9_ZRmap_SAL_rh_sm1

M9_ZRmap_MUS_rh_sm1

M9_diffZRmap_rh_clustA5_sm1
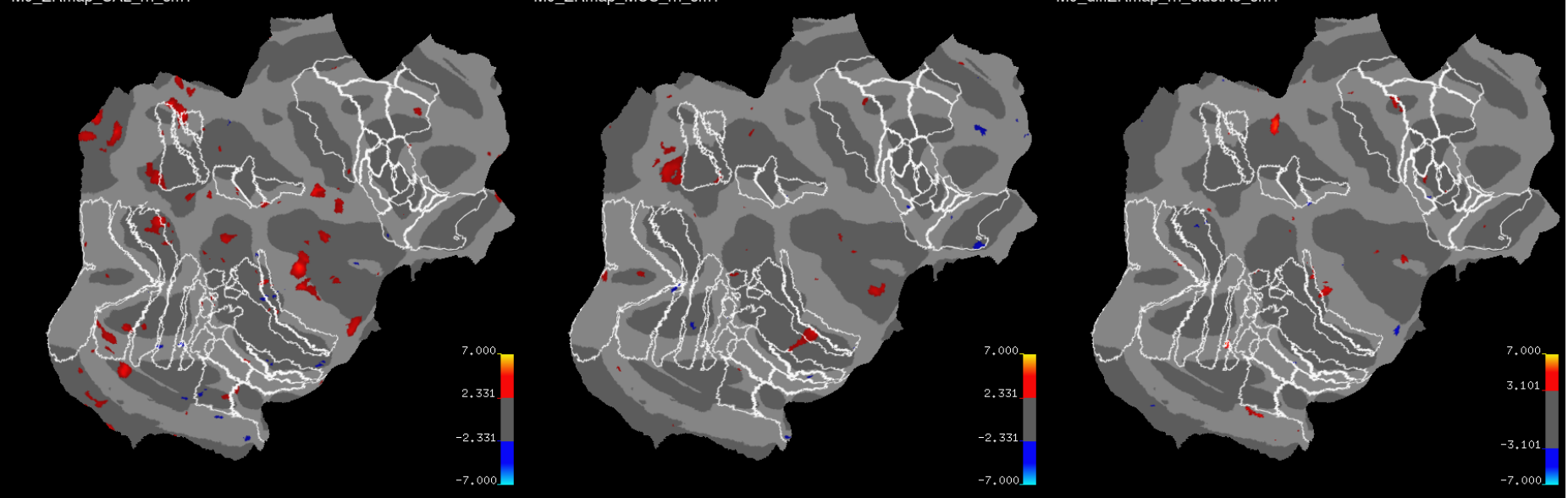

\section{Contralesional}


Figure 7S2. Behavioral correlations for saline and muscimol, and the difference between muscimol and saline for M10

\section{Ipsilesional}

Saline Muscimol

Muscimol vs. Saline

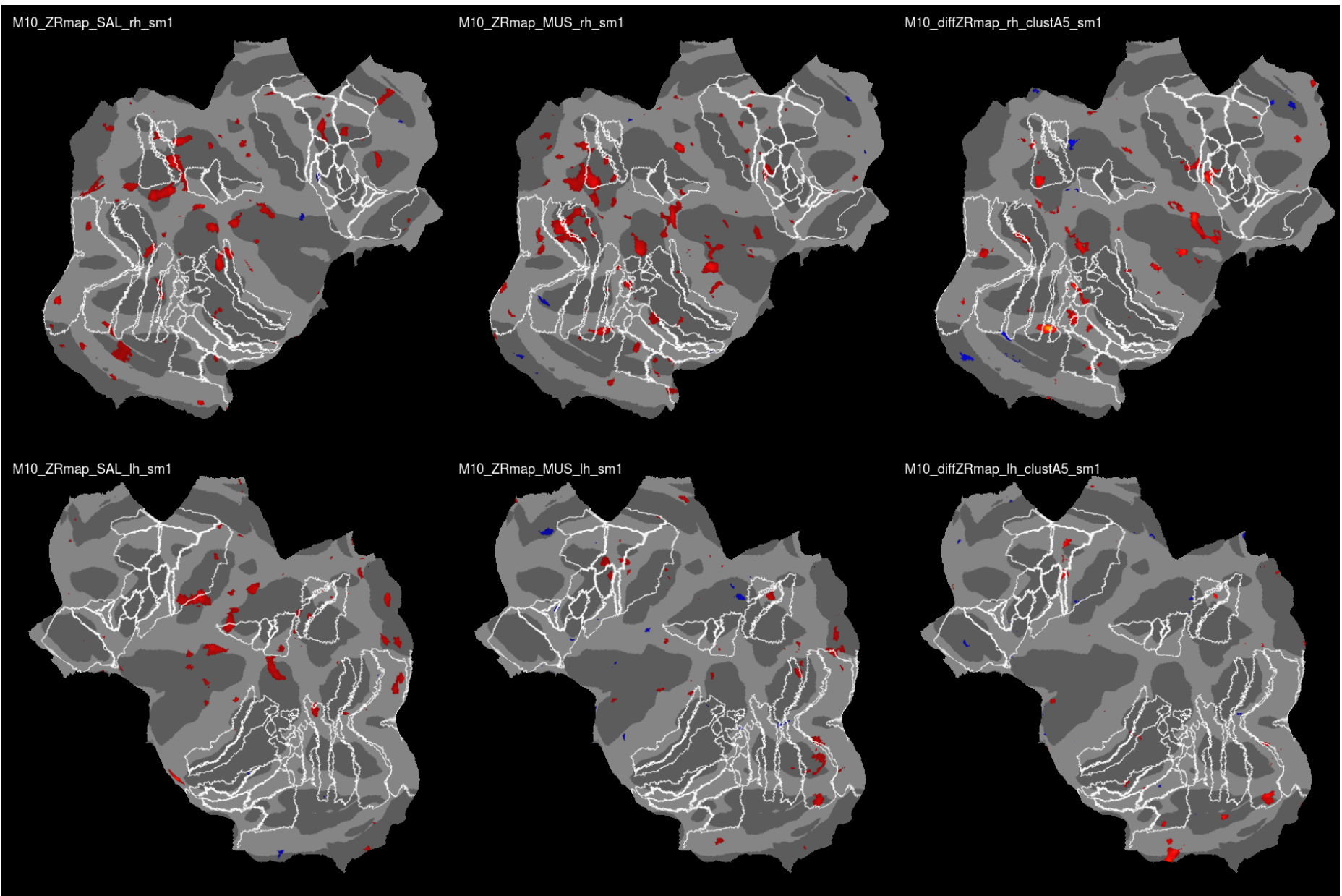

Contralesional 
Figure $8 \mathrm{~S} 1$ Similar with Fig. 8 , but is using an extended number of ROls.

A
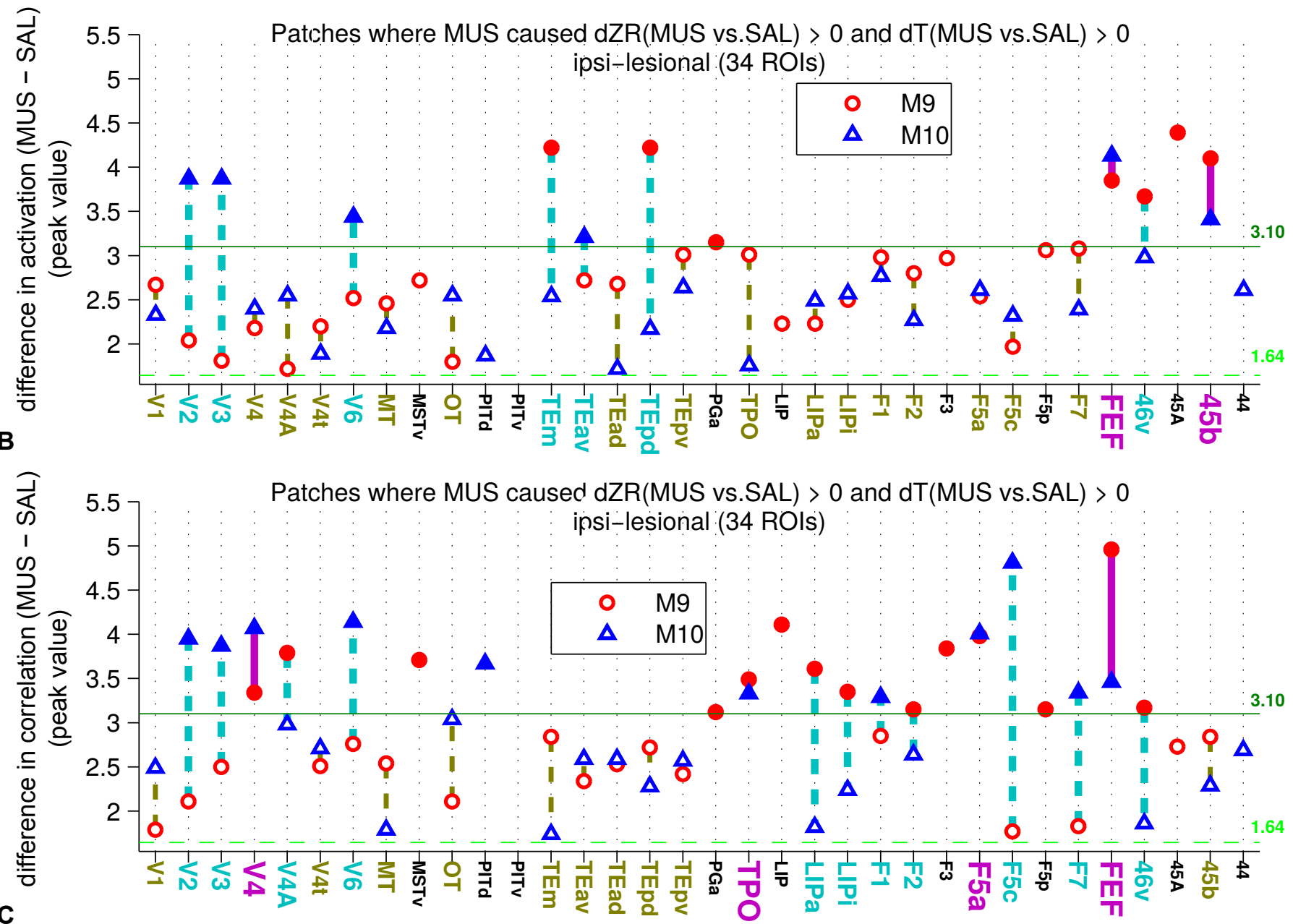

C

D

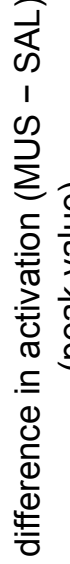

$\left.\begin{array}{r}5.5 \\ 5\end{array}\right] \quad \begin{array}{r}\text { Patches where MUS caused dZR(MUS vs.SAL) }>0 \text { and dT(MUS vs.SAL) }>0 \\ \text { contra-lesional (34 ROIs) }\end{array}$

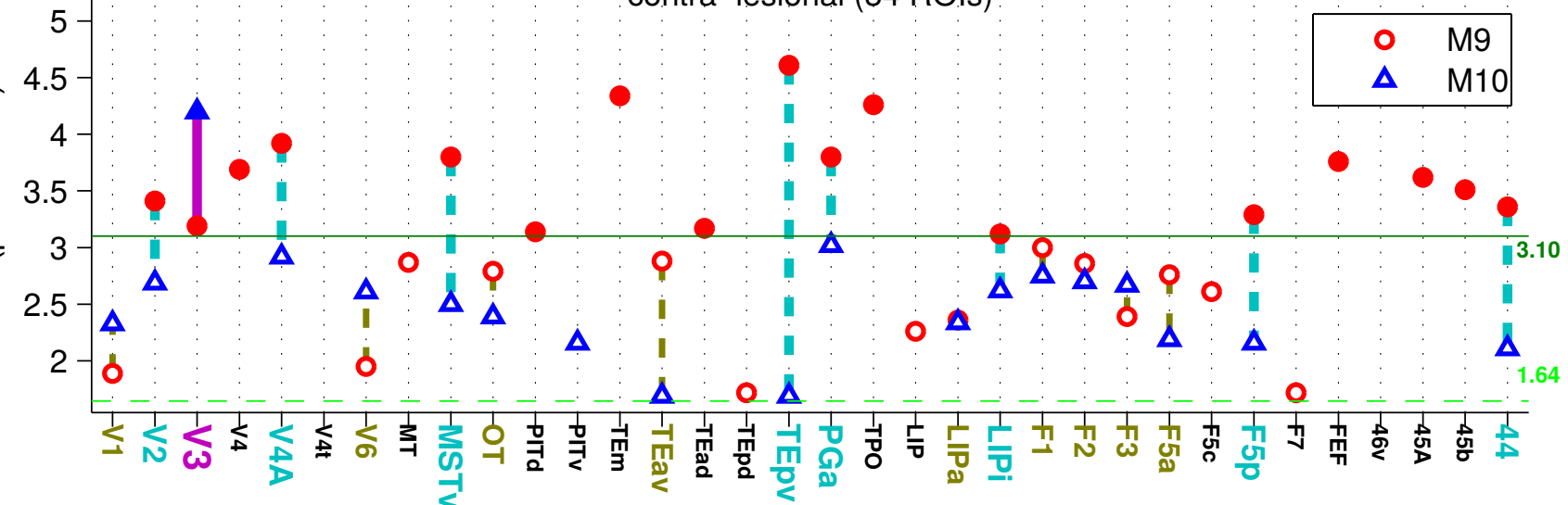

5.5 : $\quad$ Patches where MUS caused dZR(MUS vs.SAL) $>0$ and $\mathrm{dT}$ (MUS vs.SAL) $>0$ contra-lesional (34 ROIs)

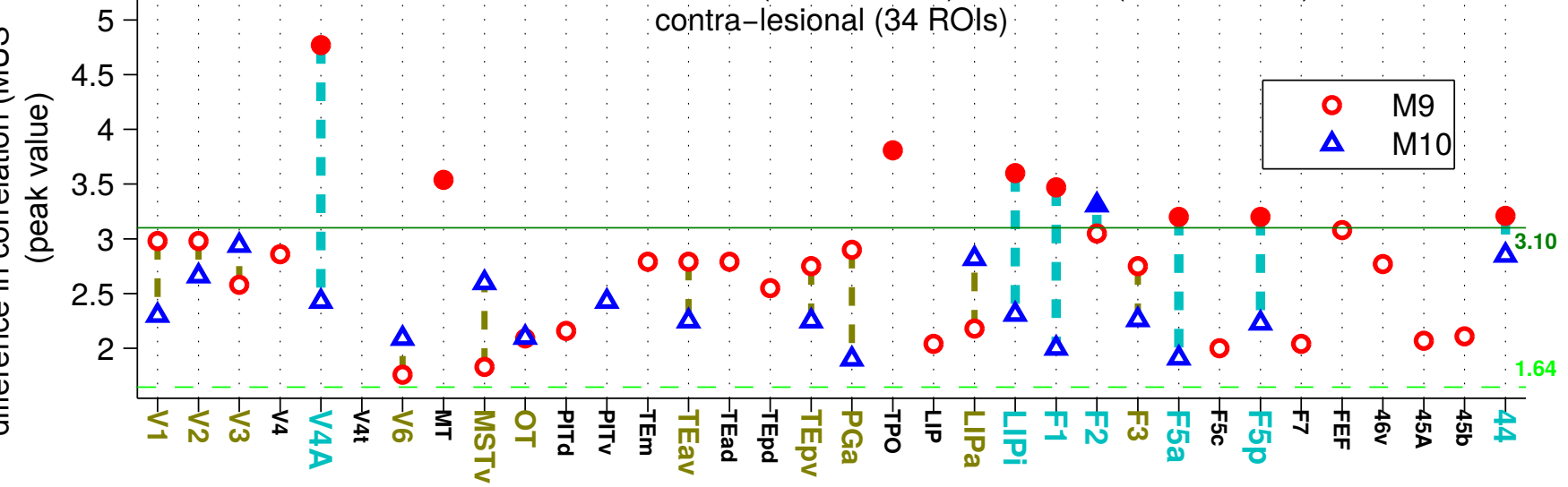


Figure 8S2. Activations (A1 - D1) and behavioral correlations (A2 - D2)

for Saline and Muscimol (MUS) separate (extended number of ROIs).

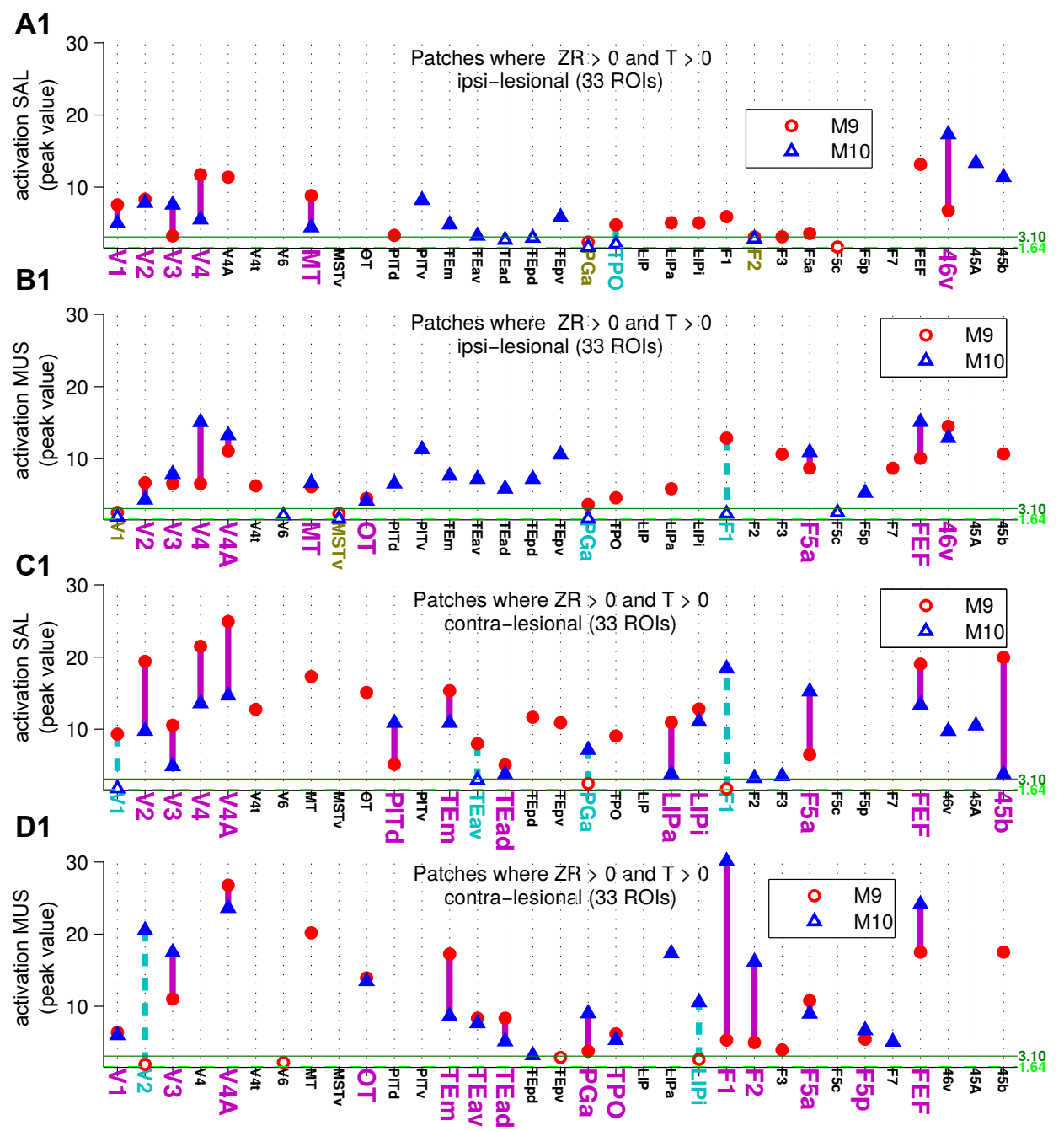

A2
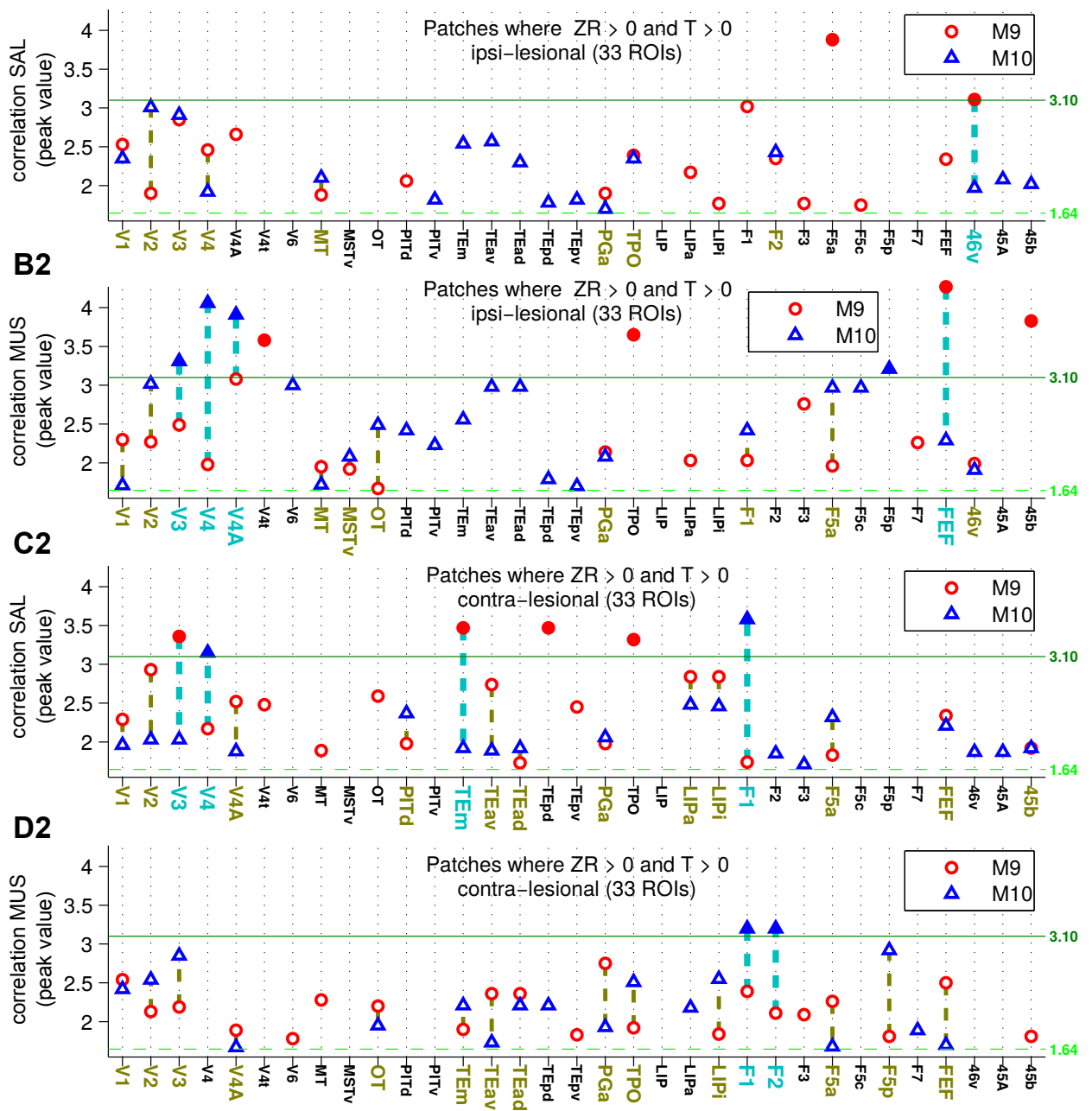
Figure 8. Differences in activation (A,C) and differences in correlation (B,D)

A

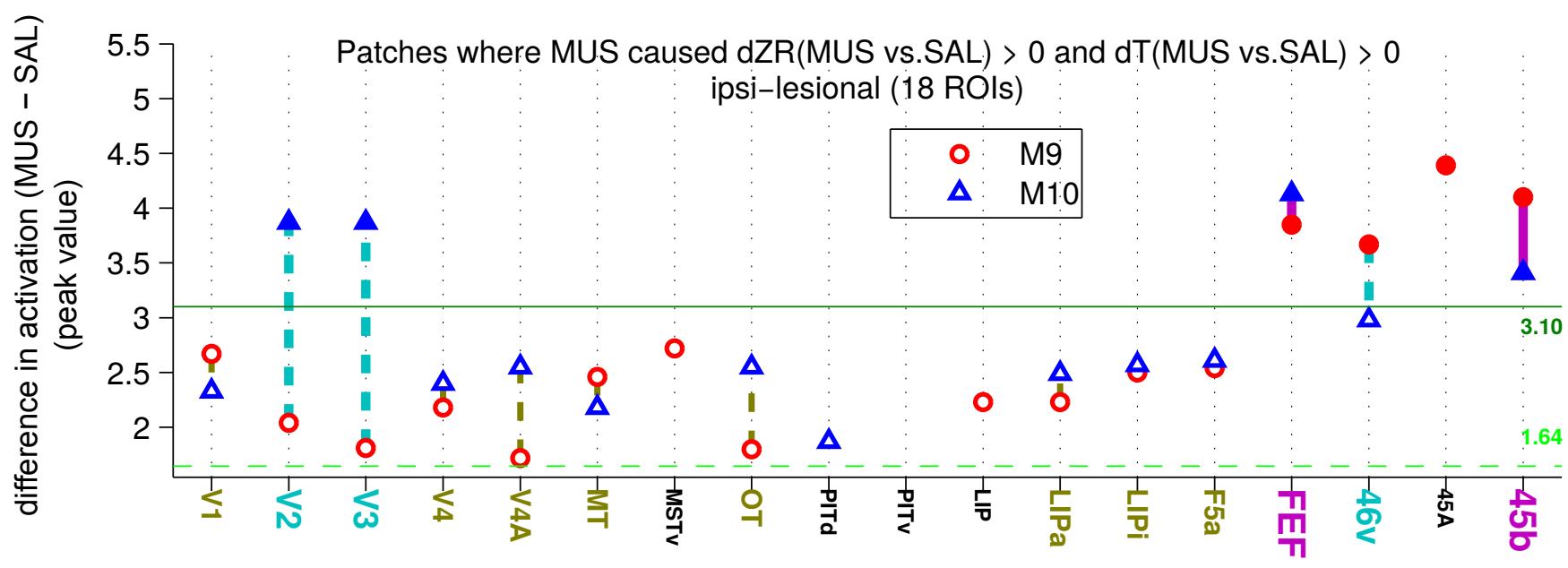

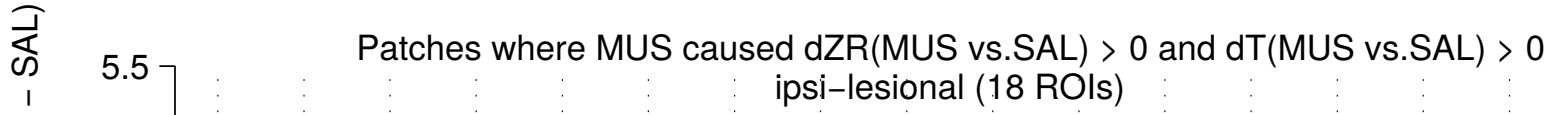
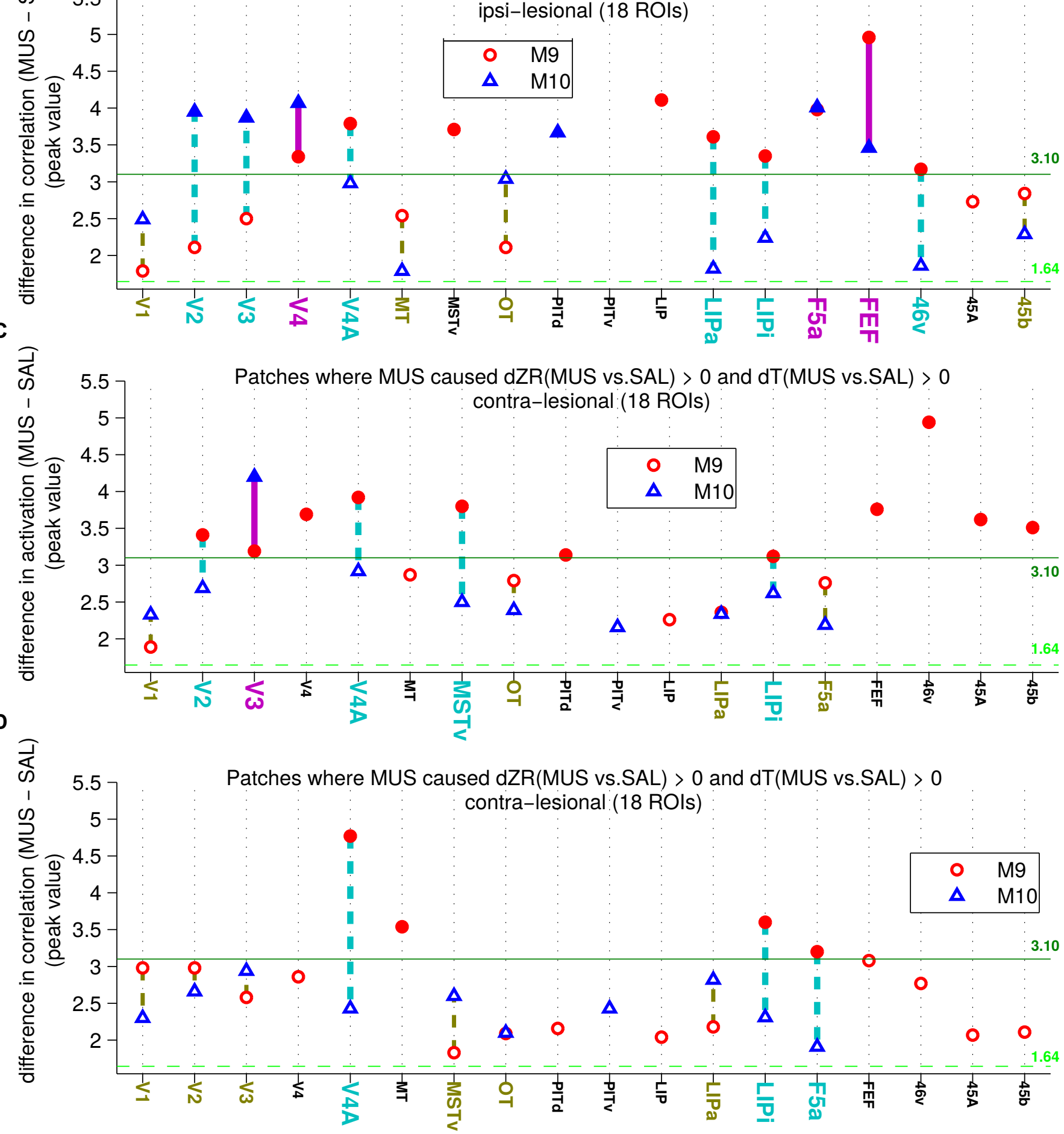